\title{
Relativistic Calculation of the Meson Spectrum: a Fully Covariant Treatment Versus Standard Treatments
}

\author{
Horace Crater \\ The University of Tennessee Space Institute Tullahoma, Tennessee 37388* \\ Peter Van Alstine \\ 12474 Sunny Glen Drive Moorpark, CA 93021
}

\begin{abstract}
A large number of treatments of the meson spectrum have been tried that consider mesons as quark - anti quark bound states. Recently, we used relativistic quantum "constraint" mechanics to introduce a fully covariant treatment defined by two coupled Dirac equations. For field-theoretic interactions, this procedure functions as a "quantum mechanical transform of Bethe-Salpeter equation". Here, we test its spectral fits against those provided by an assortment of models: Wisconsin model, Iowa State model, Brayshaw model, and the popular semi-relativistic treatment of Godfrey and Isgur. We find that the fit provided by the two-body Dirac model for the entire meson spectrum competes with the best fits to partial spectra provided by the others and does so with the smallest number of interaction functions without additional cutoff parameters necessary to make other approaches numerically tractable. We discuss the distinguishing features of our model that may account for the relative overall success of its fits. Note especially that in our approach for QCD, the resulting pion mass and associated Goldstone behavior depend sensitively on the preservation of relativistic couplings that are crucial for its success when solved nonperturbatively for the analogous two-body bound-states of QED.
\end{abstract}

\footnotetext{
*hcrater@utsi.edu
} 


\section{INTRODUCTION}

Over 50 years after the discovery of the first meson and over 25 years after the identification of its underlying quark degrees of freedom, the Strong-Interaction Bound-state problem remains unsolved. Perhaps eventually the full spectrum of mesonic and baryonic states will be calculated directly from Quantum Chromodynamics via lattice gauge theory. This would require use of techniques that were unknown to the founding fathers of QED. For the present though, researchers have had to content themselves with attempts to extend bits and pieces of traditional QED bound-state treatments into the realm of QCD. Unfortunately, for those bound systems whose constituent kinetic or potential energies are comparable to constituent rest masses, nonrelativistic techniques are inadequate from the start.

In the QED bound-state problem, weakness of the coupling permitted calculation through perturbation about the nonrelativistic quantum mechanics of the Schrödinger equation. Using the equation adopted by Breit [1]-[3] (eventually justified by the Bethe-Salpeter equation [4]), one was faced with the fact that a nonperturbative numerical treatment of the Breit

equation could not yield spectral results that agree to an appropriate order with a perturbative treatment of the semirelativistic form of that equation[4]-[9]. This form of the equation contained such terms as contact terms bred by the vector Darwin interaction that could be treated only perturbatively, spoiling the interpretation of the Breit equation as a bona-fide wave equation. Forays into the full relativistic structure defined by the Bethe-Salpeter equation turned up fundamental problems which fortunately could be sidestepped for QED due to the smallness of $\alpha$.

In the absence of definitive guidance from QED, in recent years researchers in QCD have felt free to jump off from any point that had proven historically useful in QED. Some have chosen to approach the spectrum using time-honored forms from the "relativistic correction structure" of atomic physics. Others have employed truncations of field-theoretic boundstate equations in hopes that the truncations do no violence to the dynamical structures or their relativistic transformation properties. A third set have broken away from QED by choosing to guess at "relativistic wave equations" as though such equations have no connection to field theory.

Is there another way to attack this problem? Imagine that we could replace the Schrodinger equation by a many-body relativistic Schrodinger equation or improved Breit 
equation that could be solved numerically. One would have to establish its validity by connecting it to quantum field theory, and its utility by solving it for QCD. Of course such an approach would apply equally as well to QED and so would have to recapitulate the known results of QED. (These results might reemerge in unfamiliar forms since not originating in the usual expansion about the nonrelativistic limit.)

Now, for the two-body bound-state problem, there is such an equation or rather a system of two coupled Dirac equations - for an interacting pair of relativistic spin one-half constituents. It turns out that for the two-body case, use of Dirac's constrained Hamiltonian mechanics [10]-[15] in a form appropriate for two spinning particles [18], [19] (pseudo-classical mechanics using Grassmann degrees of freedom[20],[21])leads to a consistent relativistic quantum description. In the two-body case, one may explicitly construct the covariant Center of Momentum rest frame of the interacting system. In fact, the relativistic twobody problem may be written as an effective relativistic one-body problem [12], [22],[23]. The proper formulation of this relativistic scheme requires the successful treatment of the quantum ghost states (due to the presence of the "relative time") that first appeared in Nakanishi's work on the Bethe-Salpeter equation[24].

It might seem that although fully covariant and quantum mechanically legitimate, such an approach would merely give a sophisticated method for guessing relativistic wave equations for systems of bound quarks. However, this method assumes its full power when combined with the field-theoretic machinery of the Bethe-Salpeter equation. When used with the kernel of the Bethe-Salpeter equation for QED, our approach combines weakpotential agreement with QED [25] with the nonperturbative structure of the field-theoretic eikonal approximation[26],[27]. The extra structure is automatically inherited from relativistic classical[28], [31] and quantum mechanics[27]. In QED our approach amounts to a "quantum-mechanical transform" [32],[33] of the Bethe Salpeter equation provided by two coupled Dirac equations whose fully covariant interactions are determined by QED in the Feynman Gauge[34],[25]. These "Two-Body Dirac Equations" are legitimate quantum wave equations that can be solved directly [35],[25] (without perturbation theory) whose numerical or analytic solutions automatically agree with results generated by ordinary perturbative treatment. (In our opinion the importance of this agreement cannot be overemphasized. A common fault of most of the models we discuss in this paper is that they lack such agreement. But, if a numerical approach to a two body bound state formalism when specialized 
to QED cannot reproduce the results given by its own perturbative treatment, how can one be certain that its application to highly relativistic QCD bound states will not include spurious short range contributions.)

Of course there is a fly in the ointment - but one to be expected on fundamental grounds. It turns out that the only separable interacting system as yet explicitly constructed in a canonical relativistic mechanics is the two body system. In practical terms, this means that we must confine the present treatment to the meson spectrum. So far, even the relativistic treatment of the three-body problem of QED in the constraint approach is unknown. No one has been able to produce three compatible separable Dirac equations which include not only mutual interactions but also necessary three body forces in closed form[13].

Although still considered unusual or unfamiliar by the bulk of bound-state researchers, the structures appearing in these equations may have been anticipated classically by J. L. Synge, the spin structures were introduced into QED (incorrectly) by Eddington and Gaunt [36],[37], and they have appeared in approximate forms appropriate for weak potentials in the works of Krapchev, Aneva, and Rizov[38] and of Pilkuhn[39]. Of greatest surprise but greatest value (to the authors), their perturbative weak-potential versions were uncovered in QED by J. Schwinger in his virial treatment of the positronium spectrum[40]. The associated relativistic mechanics transforms properly under spin-dependent generalizations of generators found by Pryce [42]Newton and Wigner[43]. The techniques for quantization go back to those found by Dirac[10], and applied by Regge and Hanson to the relativistic top[44], by Nambu to the string, by Galvao and Teitelboim to the single spin one-half particle [21], and by Kalb and Van Alstine [11]and by Todorov[12] to the pair of spinless particles. Their progenitors can be found in the bilocal field theories of Yukawa, Markov, Feynman and Gell-Mann as well as the myriad treatments of the relativistic oscillator beginning with the work of Schrödinger.

In this paper, we will compare our latest results for the meson spectrum provided by Two-Body Dirac Equations with the corresponding results given by a representative sample of alternative methods. The present paper is not a detailed account of this method (already presented elsewhere - see Refs.[25][45] and references contained therein). Neither is it an attempt to conduct an even-handed or thorough review. Rather, its purpose is to show how such an organized scheme fares in the real world of calculation of a relativistic spectrum and to contrast its results with those produced by an array of approaches, each chosen on account of popularity or structural resemblances or differences with our approach. In this paper we 
consider only approaches like ours that do not restrict themselves to the heavy mesons but attempt fits to the entire spectrum thus obtaining a more demanding comparison. (We do not consider here the myriad of partial spectral results for either the light or heavy mesons appearing in the recent literature). Where possible, we shall show how certain distinguishing features of the various approaches are responsible for success or failure of the resulting fits to the meson spectrum. Whether our equations ultimately prove correct or not, they have the virtue that they are explicitly numerically solvable without additional revisions, cutoffs, etc. unlike certain other approaches whose spectral consequences depend on ad hoc revision necessary for numerical solution.

All of the treatments we examine attempt to describe the interactions of QCD through the inclusion of spin-dependent interactions that in part first appeared as small corrections in atomic physics. All include relativistic kinematics for the constituents. One contributor to the use of such techniques [46] has even asserted that all of the alternative approaches that include relativistic kinematics are actually equivalent to the nonrelativistic quark model, so that the detailed relativistic structure of the interaction makes no difference to the bound state spectrum. However, as we shall see in a fully relativistic description with no extraneous parameters, the detailed relativistic interaction structure in fact determines the success or failure of a calculation of the full meson spectrum from a single equation.

The order of the paper is as follows:

First, in Section II, we review enough of the structures of our Two-Body Dirac Equations and their origins in relativistic constraint dynamics to make clear the equations that we are solving and the relativistic significances of the potential structures appearing in them. (Those readers who are already familiar with constraint dynamics might wish to go directly to the QCD applications of section III.) In Section III, we detail how we incorporate the interactions of QCD into our equations by constructing the relativistic version of the Adler-Piran static quark potential[47] that we use when we apply our equations to meson spectroscopy. In Section IV, we examine the numerical spectral results that are generated by this application of the Two-Body Dirac Equations.

The feature of our approach that most distinguishes it from other more traditional twobody formalisms is its use of two coupled constituent equations (instead of one) containing two-body minimal substitution forms and related structures that incorporate the minimal interaction form of the original one-body Dirac equation. In Section $V$ we rewite this form of 
the Two Body Dirac Equations first as an equivalent one that incorporates the interactions through the kernel structures that appear in most older approaches and second as an equivalent form closely related to the Breit equation. In this section we examine how the relativistic interaction structures of the constraint approach lead even for QED to classifications of interaction terms that differ from the designations used in some of the other approaches. In Section VI, we examine an attempt to use the Salpeter Equation to treat the meson spectrum: the Wisconsin Model of Gara, Durand, Durand, and Nickisch[48]. Although these authors try to keep relativistic structures, they ultimately employ weak-potential approximations and structures obtained from perturbative QED in a non-perturbative equation (with no check to see that the procedure even makes nonperturbative sense in QED itself). In Section VII we examine the Iowa State Model of Sommerer, Spence and Vary [49] which uses a new quasipotential approach for which, in contrast to the Wisconsin model, the authors check that the equation makes nonperturbative sense in QED at least for the positronium ground state. In Section VIII, we examine the Breit Equation Model of Brayshaw [50], which illustrates the sort of successful fit that one can still obtain when one is allowed to introduce confining interactions (into the Breit equation) through terms whose relativistic transformation properties are ambiguous. In Section IX, we look at the most popular treatment - the Semirelativistic Model of Godfrey and Isgur [51]. This model includes a different smearing and momentum dependent factor for each part of the various spin-dependent interactions. Although each interaction is introduced for apparently justifiable physical reasons, this approach breaks up (or spoils) the full relativistic spin structure that is the two-body counterpart of that of the one-body Dirac equation with its automatic relations among the various interaction terms. We examine this model to see how well our fully covariant set of two-body Dirac equations, employing only three potential parameters used in two different invariant interaction functions, can do versus Godfrey's and Isgur's semirelativistic equation with relativistic kinematics and pieces of relativistic dynamical corrections (introduced in a patchwork manner with ten potential parameters used in six different interaction functions), when required to fit the whole meson spectrum (including the light-quark mesons). Finally, in Section X, we conclude the paper by reviewing some of the features of the constraint approach that played important roles in the relative success of its fit to the meson spectrum. We then use apparent successes of recent fits produced by the ordinary nonrelativistic quark model to point out dangers inherent in judging rival formalisms on the basis of 
fits to portions of the spectrum. At the end of the paper, we supply sets of tables for spectral comparisons and appendices detailing the radial form of our Two-Body Dirac equations that we use for our spectral calculations, and the numerical procedure that we use to construct meson wave functions. We also include a table summarizing the important features of the various methods that we compare in this paper.

\section{THE TWO-BODY DIRAC EQUATIONS OF CONSTRAINT DYNAMICS}

In order to treat a single relativistic spin-one-half particle, Dirac originally constructed a quantum wave equation from a first-order wave operator that is the matrix square-root of the corresponding Klein-Gordon operator [52]. Our method extends his construction to the system of two interacting relativistic spin-one-half particles with quantum dynamics governed by a pair of compatible Dirac equations on a single 16-component wave function. For an extensive review of this approach, see Refs.[23, 25, 45] and works cited therein. For the reader unfamiliar with this approach, we present a brief review.

About 27 years ago, the relativistic constraint approach first successfully yielded a covariant yet canonical formulation of the relativistic two-body problem for two interacting spinless classical particles. It accomplished this by covariantly controlling the troublesome relative time and relative energy, thereby reducing the number of degrees of freedom of the relativistic two-body problem to that of the corresponding nonrelativistic problem[11]-[15]. In this method, the reduction takes place through the enforcement of a generalized mass shell constraint for each of the two interacting spinless particles: $p_{i}^{2}+m_{i}^{2}+\Phi_{i} \approx 0$. Mathematical consistency then requires that the two constraints be "compatible" in the sense that they be conserved by a covariant system-Hamiltonian. Upon quantization, the quantum version

of this "compatibility condition" becomes the requirement that the quantum versions of the constraints (two separate Klein-Gordon equations on the same wave function for spinless particles) possess a commutator that vanishes when applied to the wave-function. In 1982, the authors of this paper used a supersymmetric classical formulation of the single-particle Dirac equation due to Galvao and Teitelboim to successfully extend this construction to the "pseudoclassical" mechanics of two spin-one-half particles [18, 21]. Upon quantization, this scheme produces a consistent relativistic quantum mechanics for a pair of interacting fermions governed by two coupled Dirac equations. 
When specialized to the case of two relativistic spin-one-half particles interacting through four-vector and scalar potentials, the two compatible 16-component Dirac equations [23, 25, 45] take the form

$$
\begin{aligned}
& \mathcal{S}_{1} \psi=\gamma_{51}\left(\gamma_{1} \cdot\left(p_{1}-A_{1}\right)+m_{1}+S_{1}\right) \psi=0 \\
& \mathcal{S}_{2} \psi=\gamma_{52}\left(\gamma_{2} \cdot\left(p_{2}-A_{2}\right)+m_{2}+S_{2}\right) \psi=0
\end{aligned}
$$

in terms of $\mathcal{S}_{i}$ operators that in the free-particle limit become operator square roots of the Klein-Gordon operator.

The relativistic four-vector potentials $A_{i}^{\mu}$ and scalar potentials $S_{i}$ are effective constituent potentials that in either limit $m_{i} \rightarrow \infty$ go over to the ordinary external vector and scalar potentials of the light-particle's one-body Dirac equation. Note that the four-vector interactions enter through "minimal substitutions" inherited (along with the accompanying gauge structure) from the corresponding classical field theory[22, 28, 31]. The covariant spindependent terms in the constituent vector and scalar potentials (see Eq.(2.10 and Eq.(2.11) below) are recoil terms whose forms are nonperturbative consequences of the compatibility condition

$$
\left[\mathcal{S}_{1}, \mathcal{S}_{2}\right] \psi=0
$$

This condition also requires that the potentials depend on the space-like interparticle separation only through the combination

$$
x_{\perp}^{\mu}=\left(\eta^{\mu \nu}+\hat{P}^{\mu} \hat{P}^{\nu}\right)\left(x_{1}-x_{2}\right)_{\nu}
$$

with no dependence on the relative time in the c.m. frame. This separation variable is orthogonal to the total four-momentum

$$
P^{\mu}=p_{1}^{\mu}+p_{2}^{\mu} ;-P^{2} \equiv w^{2}
$$

$\hat{P}$ is the time-like unit vector

$$
\hat{P}^{\mu} \equiv P^{\mu} / w
$$

The accompanying relative four-momentum canonically conjugate to $x_{\perp}$ is

$$
p^{\mu}=\left(\epsilon_{2} p_{2}^{\mu}-\epsilon_{1} p_{2}^{\mu}\right) / w ; \text { where } \epsilon_{1}+\epsilon_{2}=w, \epsilon_{1}-\epsilon_{2}=\left(m_{1}^{2}-m_{2}^{2}\right) / w
$$

in which $w$ is the total c.m. energy. The $\epsilon_{i}$ 's are the invariant c.m. energies of each of the (interacting) particles[29]. 
The wave operators in Eqs.(2.1a,2.1b) operate on a single 16-component spinor which we decompose as

$$
\psi=\left(\begin{array}{c}
\psi_{1} \\
\psi_{2} \\
\psi_{3} \\
\psi_{4}
\end{array}\right)
$$

in which the $\psi_{i}$ are four-component spinors.

Once we have ensured that the compatibility condition is satisfied, Eqs.(2.1a,2.1b) provide a consistent quantum description of the relativistic two-body system incorporating several important properties $[23,25,45]$. They are manifestly covariant. They reduce to the ordinary one body Dirac equation in the limit in which either of the particles becomes infinitely heavy. They can be combined to give $[25,53]$ coupled second-order Schrödinger-like equations (Pauli-forms) for the sixteen component Dirac spinors. In the center of momentum (c.m.) system, for the vector and scalar interactions of Eq.(2.10) and Eq.(2.11) below, these equations resemble ordinary Schrödinger equations with interactions that include centralpotential, Darwin, spin-orbit, spin-spin, and tensor terms. These customary terms are accompanied by others that provide important additional couplings between the upper-upper $\left(\psi_{1}\right)$ and lower-lower $\left(\psi_{4}\right)$ four component spinor portions of the full sixteen component Dirac spinor. The interactions are completely local but depend explicitly on the total energy $w$ in the c.m. frame. In this paper we use a recently developed rearrangement of these equations [53] (similar to that first presented in [54]) that provides us with ones simpler to solve but physically equivalent The resulting local Schrödinger-like equation depending on the four-component spinor $\phi_{+} \equiv \psi_{1}+\psi_{4}$ takes the general c.m. form

$$
\left(-\nabla^{2}+\Phi\left(\mathbf{r}, \boldsymbol{\sigma}_{1}, \boldsymbol{\sigma}_{2}, w\right)\right) \phi_{+}=b^{2}(w) \phi_{+}
$$

with no coupling to other four component spinors. The explicit version of the potential $\Phi$ in Eq.(2.8) that results from the rearrangement has a structure that produces couplings between the spin components of $\phi_{+}$that are no more complicated than those of its nonrelativistic counterpart - with the customary spin-spin, spin-orbit, non-central tensor or spin-orbit difference terms appearing. We have checked that both the simpler form Eq.(2.8) and the equivalent coupled forms give the same numerical spectral results when tested for QED bound states as in [25] and when tested for our new QCD spectral results appearing in this 
paper. (This provides an important cross check on our numerical calculation of the meson spectra). Eq.(2.8) is accompanied by similar equations for $\phi_{-} \equiv \psi_{1}-\psi_{4}$ and $\chi_{ \pm} \equiv \psi_{2} \pm \psi_{3}$. Once Eq.(2.8) is solved, one can use Eq.(2.1a,2.1b) to determine $\phi_{-}$and $\chi_{ \pm}$. Because of the decoupling it is not necessary to determine $\phi_{-}$and $\chi_{ \pm}$to solve the eigenvalue equation (2.8). However, the detailed form of $\Phi$ for $\phi_{+}$results from their elimination through the Pauli reduction procedure. In these equations, the usual invariant

$$
b^{2}(w) \equiv\left(w^{4}-2 w^{2}\left(m_{1}^{2}+m_{2}^{2}\right)+\left(m_{1}^{2}-m_{2}^{2}\right)^{2}\right) / 4 w^{2}
$$

plays the role of energy eigenvalue. This invariant is the c.m. value of the square of the relative momentum expressed as a function of the invariant total c.m. energy $w$.

Note that in the limit in which one of the particles becomes very heavy, this Schrödingerlike equation turns into the one obtained by eliminating the lower component of the ordinary one-body Dirac equation in terms of the other component.

The vector potentials appearing in Eqs.(2.1a,2.1b) depend on three invariant functions $E_{1}, E_{2}$, and $G$ that define time-like vector interactions (proportional to $\hat{P}$ ) and space-like vector interactions (orthogonal to $\hat{P}$, with $\partial_{\mu} \equiv \partial / \partial x^{\mu}$ ) [23, 25]

$$
\begin{aligned}
& A_{1}^{\mu}=\left(\left(\epsilon_{1}-E_{1}\right)-i \frac{G}{2} \gamma_{2} \cdot\left(\frac{\partial E_{1}}{E_{2}}+\partial G\right) \gamma_{2} \cdot \hat{P}\right) \hat{P}^{\mu}+(1-G) p^{\mu}-\frac{i}{2} \partial G \cdot \gamma_{2} \gamma_{2}^{\mu} \\
& A_{2}^{\mu}=\left(\left(\epsilon_{2}-E_{2}\right)+i \frac{G}{2} \gamma_{1} \cdot\left(\frac{\partial E_{2}}{E_{1}}+\partial G\right) \gamma_{1} \cdot \hat{P}\right) \hat{P}^{\mu}-(1-G) p^{\mu}+\frac{i}{2} \partial G \cdot \gamma_{1} \gamma_{1}^{\mu},
\end{aligned}
$$

while the scalar potentials $S_{i}$ depend on $G$ and two additional invariant functions $M_{1}$ and $M_{2}$

$$
\begin{aligned}
S_{1} & =M_{1}-m_{1}-\frac{i}{2} G \gamma_{2} \cdot \frac{\partial M_{1}}{M_{2}} \\
S_{2} & =M_{2}-m_{2}+\frac{i}{2} G \gamma_{1} \cdot \frac{\partial M_{2}}{M_{1}} .
\end{aligned}
$$

Note that the terms in 2.10 and 2.11 which are explicitly spin-dependent through the gamma matrices are essential in order to satisfy the compatibility condition 2.2. Later on, when the equation is reduced to second-order "Pauli-form", yet other spin dependences eventually arise from gamma matrix terms (that, when squared, lose their gamma matrix dependence). These are typical of what occurs in the reduction of the one-body Dirac equation to the "Pauli form". The gamma matrices also give rise to spin independent terms in the Pauliforms. These terms emerge in a manner similar to the above two sources of spin dependent terms in the Pauli-form of the equations. 
In the case in which the space-like and time-like vectors are not independent but combine into electromagnetic-like four-vectors, the constituent vector interactions appear in a more compact form

$$
\begin{aligned}
& A_{1}^{\mu}=\left(\epsilon_{1}-\frac{G\left(\epsilon_{1}-\epsilon_{2}\right)}{2}+\frac{\left(\epsilon_{1}-\epsilon_{2}\right)}{2 G}\right) \hat{P}^{\mu}+(1-G) p^{\mu}-\frac{i}{2} \partial G \cdot \gamma_{2} \gamma_{2}^{\mu} \\
& A_{2}^{\mu}=\left(\epsilon_{2}-\frac{G\left(\epsilon_{2}-\epsilon_{1}\right)}{2}+\frac{\left(\epsilon_{1}-\epsilon_{2}\right)}{2 G}\right) \hat{P}^{\mu}-(1-G) p^{\mu}+\frac{i}{2} \partial G \cdot \gamma_{1} \gamma_{1}^{\mu} .
\end{aligned}
$$

In that case $E_{1}, E_{2}$ and $G$ are related to each other[22,23] $\left(\partial E_{1} / E_{2}=-\partial \log G\right)$ and for our QCD applications (as well as for QED) are functions of only one invariant function $\mathcal{A}(r)$ in which $r$ is the invariant

$$
r \equiv \sqrt{x_{\perp}^{2}} .
$$

They take the forms

$$
\begin{aligned}
& E_{1}^{2}(\mathcal{A})=G^{2}\left(\epsilon_{1}-\mathcal{A}\right)^{2}, \\
& E_{2}^{2}(\mathcal{A})=G^{2}\left(\epsilon_{2}-\mathcal{A}\right)^{2},
\end{aligned}
$$

in which

$$
G^{2}=\frac{1}{(1-2 \mathcal{A} / w)} .
$$

In the forms of these equations used below, Todorov's collective energy variable

$$
\epsilon_{w}=\left(w^{2}-m_{1}^{2}-m_{2}^{2}\right) / 2 w,
$$

will eventually appear.

In general $M_{1}$ and $M_{2}$ are related to each other[18, 23] and for QCD applications are functions of two invariant functions $\mathcal{A}(r)$ and $S(r)$ appearing in the forms:

$$
\begin{aligned}
& M_{1}^{2}(\mathcal{A}, S)=m_{1}^{2}+G^{2}\left(2 m_{w} S+S^{2}\right) \\
& M_{2}^{2}(\mathcal{A}, S)=m_{2}^{2}+G^{2}\left(2 m_{w} S+S^{2}\right),
\end{aligned}
$$

in which

$$
m_{w}=m_{1} m_{2} / w .
$$

(In these equations, $m_{w}$ and $\epsilon_{w}$ are the relativistic reduced mass and energy of the fictitious particle of relative motion introduced by Todorov $[12,26]$, which satisfy the effective onebody Einstein condition

$$
\epsilon_{w}^{2}-m_{w}^{2}=b^{2}(w) .
$$


In the limit in which one of the particles becomes infinitely heavy, $m_{w}$ and $\epsilon_{w}$ reduce to the mass and energy of the lighter particle.) The invariant function $S(r)$ is primarily responsible for the constituent scalar potentials since $S_{i}=0$ if $S(r)=0$, while $\mathcal{A}(r)$ contributes to the $S_{i}$ (if $S(r) \neq 0$ ) as well as to the vector potentials $A_{i}^{\mu}$. Originally, we derived the general forms of Eqs. $(2.17,2.14,2.15)$ for the scalar and vector potentials using classical field theoretic arguments $[28,31]$ (see also [18, 26]). Surprisingly, the resulting forms for the mass and energy potential functions $M_{i}, G$ and $E_{i}$ automatically embody collective minimal substitution rules for the spin-independent parts of the Schrödinger-like forms of the equations. Classically those forms turn out to be modifications of the Einstein condition for the free effective particle of relative motion

$$
p^{2}+m_{w}^{2}=\epsilon_{w}^{2}
$$

For the vector interaction they automatically generate the replacement of $\epsilon_{w}$ by $\epsilon_{w}-\mathcal{A}$ and for the scalar interaction the replacement of $m_{w}$ by $m_{w}+S$. The part of Eq.(2.8) that results from the vector and scalar interactions then takes the form

$$
\left(p^{2}+2 m_{w} S+S^{2}+2 \epsilon_{w} \mathcal{A}-\mathcal{A}^{2}\right) \phi_{+}=b^{2} \phi_{+}
$$

Now, we originally found these forms starting from relativistic classical field theory. The deceptively simple form of Eq.(2.21) in fact incorporates retarded and advanced effects through its dependnce on the c.m. energy $w$. On the other hand, recently Jallouli and Sazdjian [27] obtained Eqs.(2.14) and (2.17) in quantum field theory after performing a necessarily laborious Eikonal summation to all orders of ladder and cross ladder diagrams together with all constraint diagrams (Lippmann-Schwinger like iterations of the simple Born diagram)[55]. Thus, the structure first discovered simply in the correspondence limit has now been verified through direct but difficult derivation from perturbative quantum field theory.

These equations contain an important hidden hyperbolic structure (which we could have used to introduce the interactions in the first place). To employ it we introduce two independent invariant functions $L\left(x_{\perp}\right)$ and $\mathcal{G}\left(x_{\perp}\right)$, in terms of which the invariant functions of Eqs.(2.10,2.11) take the forms:

$$
\begin{aligned}
& M_{1}=m_{1} \cosh L+m_{2} \sinh L \\
& M_{2}=m_{2} \cosh L+m_{1} \sinh L
\end{aligned}
$$




$$
\begin{gathered}
E_{1}=\epsilon_{1} \cosh \mathcal{G}-\epsilon_{2} \sinh \mathcal{G} \\
E_{2}=\epsilon_{2} \cosh \mathcal{G}-\epsilon_{1} \sinh \mathcal{G} \\
G=\exp \mathcal{G} .
\end{gathered}
$$

In terms of $\mathcal{G}$ and the constituent momenta $p_{1}$ and $p_{2}$, the individual four-vector potentials of Eq.(2.14) take the suggestive forms

$$
\begin{aligned}
& A_{1}=[1-\cosh (\mathcal{G})] p_{1}+\sinh (\mathcal{G}) p_{2}-\frac{i}{2}\left(\partial \exp \mathcal{G} \cdot \gamma_{2}\right) \gamma_{2} \\
& A_{2}=[1-\cosh (\mathcal{G})] p_{2}+\sinh (\mathcal{G}) p_{1}+\frac{i}{2}\left(\partial \exp \mathcal{G} \cdot \gamma_{1}\right) \gamma_{1}
\end{aligned}
$$

Eqs.(2.22), (2.23) and (2.25) together display a further consequence of the compatibility condition, a kind of relativistic version of Newton's third law in the sense that the two sets of constituent scalar and vector potentials are each given in terms of just one invariant function, $S$ and $\mathcal{A}$ respectively.

In terms of these functions the coupled two-body Dirac equations then take the form

$$
\begin{aligned}
& \mathcal{S}_{1} \psi=\left(-G \beta_{1} \Sigma_{1} \cdot \mathcal{P}_{2}+E_{1} \beta_{1} \gamma_{51}+M_{1} \gamma_{51}-G \frac{i}{2} \Sigma_{2} \cdot \partial\left(\mathcal{G} \beta_{1}+L \beta_{2}\right) \gamma_{51} \gamma_{52}\right) \psi=0 \\
& \mathcal{S}_{2} \psi=\left(G \beta_{2} \Sigma_{2} \cdot \mathcal{P}_{1}+E_{2} \beta_{2} \gamma_{52}+M_{2} \gamma_{52}+G \frac{i}{2} \Sigma_{1} \cdot \partial\left(\mathcal{G} b_{2}+L \beta_{1}\right) \gamma_{51} \gamma_{52}\right) \psi=0
\end{aligned}
$$

in which

$$
\mathcal{P}_{i} \equiv p-\frac{i}{2} \Sigma_{i} \cdot \partial \mathcal{G} \Sigma_{i}
$$

depending on gamma matrices with block forms

$$
\begin{aligned}
& \beta_{1}=\left(\begin{array}{cc}
1_{8} & 0 \\
0 & -1_{8}
\end{array}\right), \quad \gamma_{51}=\left(\begin{array}{cc}
0 & 1_{8} \\
1_{8} & 0
\end{array}\right), \quad \beta_{1} \gamma_{51} \equiv \rho_{1}=\left(\begin{array}{cc}
0 & 1_{8} \\
-1_{8} & 0
\end{array}\right) \\
& \beta_{2}=\left(\begin{array}{ll}
\beta & 0 \\
0 & \beta
\end{array}\right), \beta=\left(\begin{array}{cc}
1_{4} & 0 \\
0 & -1_{4}
\end{array}\right) \\
& \gamma_{52}=\left(\begin{array}{cc}
\gamma_{5} & 0 \\
0 & \gamma_{5}
\end{array}\right), \gamma_{5}=\left(\begin{array}{cc}
0 & 1_{4} \\
1_{4} & 0
\end{array}\right)
\end{aligned}
$$

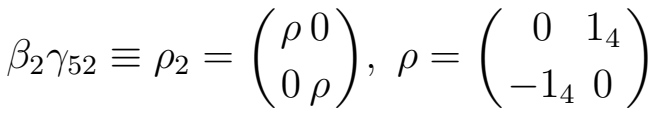

$$
\begin{aligned}
& \beta_{1} \gamma_{51} \gamma_{52}=\left(\begin{array}{cc}
0 & \gamma_{5} \\
-\gamma_{5} & 0
\end{array}\right) \\
& \beta_{2} \gamma_{52} \gamma_{51}=\left(\begin{array}{ll}
0 & \rho \\
\rho & 0
\end{array}\right) \text {. }
\end{aligned}
$$




$$
\Sigma_{i}=\gamma_{5 i} \beta_{i} \gamma_{\perp i}
$$

As described in Appendix A, a procedure analogous to the Pauli reduction procedure of the one-body Dirac equation case yields

$$
\begin{gathered}
{\left[p^{2}+2 m_{w} S+S^{2}+2 \epsilon_{2} \mathcal{A}-\mathcal{A}^{2}\right.} \\
-\left[2 \mathcal{G}^{\prime}-\frac{E_{2} M_{2}+E_{1} M_{1}}{E_{2} M_{1}+E_{2} M_{1}}(L-\mathcal{G})^{\prime}\right] i \hat{r} \cdot p-\frac{1}{2} \nabla^{2} \mathcal{G}-\frac{1}{4}(\mathcal{G})^{\prime 2}-\left(\mathcal{G}^{\prime}+L^{\prime}\right)^{2}+\frac{E_{2} M_{2}+E_{1} M_{1}}{E_{2} M_{1}+E_{2} M_{1}} \frac{1}{2} \mathcal{G}^{\prime}\left(L-\mathcal{G}^{\prime}\right) \\
+\frac{L \cdot\left(\sigma_{1}+\sigma_{2}\right)}{r}\left[\mathcal{G}^{\prime}-\frac{1}{2} \frac{E_{2} M_{2}+E_{1} M_{1}}{E_{2} M_{1}+E_{2} M_{1}}(L-\mathcal{G})^{\prime}\right]-\frac{L \cdot\left(\sigma_{1}-\sigma_{2}\right)}{2 r} \frac{E_{2} M_{2}-E_{1} M_{1}}{E_{2} M_{1}+E_{2} M_{1}}(L-\mathcal{G})^{\prime} \\
+\sigma_{1} \cdot \sigma_{2}\left(\frac{1}{2} \nabla^{2} \mathcal{G}+\frac{1}{2 r} L^{\prime}+\frac{1}{2}\left(\mathcal{G}^{\prime}\right)^{2}-\frac{1}{2} \mathcal{G}^{\prime}(L-\mathcal{G})^{\prime} \frac{E_{2} M_{2}+E_{1} M_{1}}{E_{2} M_{1}+E_{2} M_{1}}\right) \\
+\sigma_{1} \cdot \hat{r} \sigma_{2} \cdot \hat{r}\left(\frac{1}{2} \nabla^{2} L-\frac{3}{2 r} L^{\prime}+\mathcal{G}^{\prime} L^{\prime}-\frac{1}{2} L^{\prime}(L-\mathcal{G})^{\prime} \frac{E_{2} M_{2}+E_{1} M_{1}}{E_{2} M_{1}+E_{2} M_{1}}\right) \\
\left.+\frac{i}{2}(L+\mathcal{G})^{\prime}\left(\sigma_{1} \cdot \hat{r} \sigma_{2} \cdot p+\sigma_{2} \cdot \hat{r} \sigma_{1} \cdot p\right)+\frac{i}{2}(L-\mathcal{G}) \frac{E_{1} M_{2}-E_{2} M_{1}}{E_{2} M_{1}+E_{2} M_{1}} \frac{\left(\sigma_{1} \times \sigma_{2}\right)}{r}\right] \phi_{+} \\
=b^{2}(w) \phi_{+}
\end{gathered}
$$

Eq.(2.35) is the coupled four-component Schrödinger-like form of our equations that we use for our quark model bound state calculations for the mesons in the present paper. It can be solved nonperturbatively not only for quark model calculations but also for QED calculations since in that case every term is quantum-mechanically well defined (less singular than $\left.-1 / 4 r^{2}\right)$.

From this equation we obtain two coupled radial Schrödinger-like equations in the general case. But for $j=0$ or spin singlet states these equations reduce to uncoupled equations. The extra component for the general case arises from orbital angular momentum mixing or spin mixing, the latter absent for equal mass states. The detailed radial forms of these equations are given in Appendix A. For the case of QED $(S=0, \mathcal{A}=-\alpha / r)$, we have solved these coupled Schrödinger-like equations numerically obtaining results that are explicitly accurate through order $\alpha^{4}$ (with errors on the order of $\alpha^{6}$ )[25]. We have even obtained analytic solutions to the full system of coupled 16 component Dirac equations in the important case of spin-singlet positronium [35]. For both numerical and analytic solution, the results agree with those produced by perturbative treatment of these equations and with standard spectral results [56]. 


\section{MESON SPECTROSCOPY}

We use the constraint Eq.(2.35) to construct a relativistic naive quark model by choosing the two invariant functions $\mathcal{G}$ and $L$ or equivalently $\mathcal{A}$ and $S$ to incorporate a version of the static quark potential originally obtained from QCD by Adler and Piran [47] through a nonlinear effective action model for heavy quark statics. They used the renormalization group approximation to obtain both total flux confinement and a linear static potential at large distances. Their model uses nonlinear electrostatics with displacment and electric fields related through a nonlinear constitutive equation with the effective dielectric constant given by a leading log log model which fixes all parameters in their model apart from a mass

scale $\Lambda$. Their static potential contains an infinite additve constant which in turn results in the inclusion of an unknown constant $U_{0}$ in the final form of their potential (hereafter called $\left.V_{A P}(r)\right)$. We insert into Eq.(2.35) invariants $\mathcal{A}$ and $S$ with forms determined so that the sum $\mathcal{A}+S$ appearing as the potential in the nonrelativistic limit of our equations becomes the Adler-Piran nonrelativistic $Q \bar{Q}$ potential (which depends on two parameters $\Lambda$ and $U_{0}$ ) plus the Coulomb interaction between the quark and antiquark. That is,

$$
V_{A P}(r)+V_{\text {coul }}=\Lambda\left(U(\Lambda r)+U_{0}\right)+\frac{e_{1} e_{2}}{r}=\mathcal{A}+S
$$

As determined by Adler and Piran, the short and long distance behaviors of $U(\Lambda r)$ generate known lattice and continuum results through the explicit appearance of an effective running coupling constant in coordinate space. That is, the Adler-Piran potential incorporates asymptotic freedom through

$$
\Lambda U(\Lambda r<<1) \sim 1 /(r \ln \Lambda r)
$$

and linear confinement through

$$
\Lambda U(\Lambda r>>1) \sim \Lambda^{2} r
$$

The long distance $(\equiv \Lambda r>2)$ behavior of the static potential $V_{A P}(r)$ is given explicitly by

$$
\Lambda\left(c_{1} x+c_{2} \ln (x)+\frac{c_{3}}{\sqrt{x}}+\frac{c_{4}}{x}+c_{5}\right)
$$

in which $x=\Lambda r$ while the coefficients $c_{i}$ are given by the Adler-Piran leading log-log model [47]. In addition to obtaining these analytic forms for short and long distances they converted 
the numerically obtained values of the potential at intermediate distances to a compact analytic expression. The nonrelativistic analysis used by Adler and Piran, however, does not determine the relativistic transformation properties of the potential. How this potential is apportioned between vector and scalar is therefore somewhat, although not completely, arbitrary. In earlier work [34] we divided the potential in the following way among three relativistic invariants $\mathcal{V}(r), S$, and $\mathcal{A}$.(In our former construction, the additional invariant $\mathcal{V}$ was responsible for a possible independent time-like vector interaction.)

$$
\begin{aligned}
& S=\eta\left(\Lambda\left(c_{1} x+c_{2} \ln (x)+\frac{c_{3}}{\sqrt{x}}+c_{5}+U_{0}\right)\right. \\
& \mathcal{V}=(1-\eta)\left(\Lambda\left(c_{1} x+c_{2} \ln (x)+\frac{c_{3}}{\sqrt{x}}+c_{5}+U_{0}\right),\right. \\
& \mathcal{A}=V_{A}-S-\mathcal{V},
\end{aligned}
$$

in which $\eta=\frac{1}{2}$. That is, we assumed that (with the exception of the Coulomb-like term $\left.\left(c_{4} / x\right)\right)$ the long distance part was equally divided between scalar and a proposed timelike vector. In the present paper we drop the time-like vector for reasons detailed below and assume instead that the scalar interaction is solely responsible for the long distance confining terms $(\eta=1)$. The attractive $\left(c_{4}=-0.58\right)$ QCD Coulomb-like portion (not to be confused with the electrostatic $V_{\text {coul }}$ ) is assigned completely to the "electromagnetic-like" part $\mathcal{A}$. That is, the constant portion of the running coupling constant corresponding to the exchange diagram is expected to be electromagnetic-like.

Elsewhere we have treated another model explicitly containing these features: the Richardson potential. Its momentum space form

$$
\tilde{V}(\vec{q}) \sim 1 / \mathbf{q}^{2} \ln n\left(1+\mathbf{q}^{2} / \Lambda^{2}\right)
$$

interpolates in a simple way between asymptotic freedom $\tilde{V}(\vec{q}) \sim 1 / \mathbf{q}^{2} \ln \left(\mathbf{q}^{2} / \Lambda^{2}\right)$ and linear confinement $\tilde{V}(\mathbf{q}) \sim 1 / \mathbf{q}^{4}$. Even though the Richardson potential is not tied to any field theoretic base in the intermediate region (unlike the Adler-Piran potential) and does not give as good fits to the data, it does provide a convenient form for displaying our points about the static quark potential. The Richardson radial form is

$$
V(r)=8 \pi \Lambda^{2} r / 27-8 \pi f(\Lambda r) /(27 r)
$$


For $r \rightarrow 0, f(\Lambda r) \rightarrow-1 / \ln (\Lambda r)$, while for $r \rightarrow \infty, f(\Lambda r) \rightarrow 1$. Thus, in this model, if the confining part of the potential is a world scalar, then in the large $r$ limit the remaining portion, regarded as an electromagnetic-like interaction corresponding to our invariant function $\mathcal{A}(r)$, would be an attractive $1 / r$ potential with a coupling constant on the order of 1 . This is in reasonable agreement with the Adler model which also has an attractive $1 / r$ part. Support for the assumption that the $c_{4}$ term belongs only to $\mathcal{A}$ also arises from phenomenological considerations. We find that attempts to assign the $c_{4}$ term to the scalar potential have a drastic effect on the spin-spin and spin-orbit splittings. In fact, using this term in $S$ through Eqs.(2.17) generates spin-spin and spin-orbit splittings that are much too small.

In our previous work, we divided the confining part equally between scalar and time-like vector so that the spin-orbit multiplets would not be inverted. This was done in order to obtain from our model the $a_{0}(980)$ meson which was then considered as the prime candidate for the relativistic counterpart of the ${ }^{3} P_{0}$ meson. However, recent analysis indicates that that meson may be instead a meson-meson or four quark bound state (see however, [59], which even interprets the $a_{0}(980)$ meson as part of a new scalar $\left({ }^{1} S_{0}\right)$ meson $q \bar{q}$ multiplet outside of the usual quark model) while a meson with mass of $1450 \mathrm{MeV}$ may be the correct candidate for the quark model state [57]. Interpretation of this other state as the ${ }^{3} P_{0}$ meson would in fact require a partial inversion of the spin-orbit triplet (from what one would expect based on the positronium analog). This partial inversion is consistent with the ${ }^{3} P_{0}$ candidate for the $u \bar{s}$ system also appearing in a position that partially inverts the spin-orbit splitting. Since the sole purpose of including $\mathcal{V}$ in our previous treatment was to prevent the inversion, we exclude it from our present treatment. In our older treatment [34], we neglected the tensor coupling, unequal mass spin-orbit difference couplings, and the $u-d$ quark mass differences. In the present treatment, we treat the entire interaction present in our equations, thereby keeping each of these effects. In our former treatment we also performed a decoupling between the upper-upper and lower-lower components of the wave functions for spin-triplet states which turned out to be defective but which we subsequently corrected in our numerical test of our formalism for QED [25]. The corrected decoupling (appearing in Eq.(2.35)) is included in the new meson calculations appearing in this paper.

In the present investigation, we compute the best fit meson spectrum for the following 
apportionment of the Adler-Piran potential:

$$
\begin{gathered}
\left.\mathcal{A}=\exp (-\beta \Lambda r)\left[V_{A P}-\frac{c_{4}}{r}\right)\right]+\frac{c_{4}}{r}+\frac{e_{1} e_{2}}{r} \\
S=V_{A P}+\frac{e_{1} e_{2}}{r}-\mathcal{A}=\left(V_{A P}-\frac{c_{4}}{r}\right)(1-\exp (-\beta \Lambda r))
\end{gathered}
$$

In order to covariantly incorporate the Adler-Piran potential into our equations, we treat the short distance portion as purely electromagnetic-like (in the sense of the transformation properties of the potential). Through the additional parameter $\beta$, the exponential factor gradually turns off the electromagnetic-like contribution to the potential at long distance except for the $1 / r$ portion mentioned above, while the scalar portion gradually turns on, becoming fully responsible for the linear confining and subdominant terms at long distance. Altogether our two invariant potential functions depend on three parameters: $\Lambda, U_{0}$, and $\beta$.

When inserted into the constraint equations, $S$ and $\mathcal{A}$ become relativistic invariant functions of the invariant separation $r=\sqrt{x_{\perp}^{2}}$. The covariant structures of the constraint formalism then embellish the central static potential with accompanying spin-dependent and recoil terms.

In general applications of these two-body Dirac equations one must ensure that the values assumed by $\mathcal{A}$ and $S$ always result in real interaction functions $E_{i}, M_{i}$, and $G$ while preserving the correct heavy particle limits. In particular a large repulsive $\mathcal{A}$ will give an imaginary $G$ while a large attractive $S$ would lead in the limit when one particle becomes heavy to an incorrect form of the one-body Dirac equation ( for $m_{2} \rightarrow \infty$ the interaction mass potential function $M_{1} \rightarrow\left|m_{1}+S\right|$ instead of $\left.m_{1}+S\right)$. In the calculations contained in the present paper, the best fit parameters turn out to be such that $\mathcal{A}$ always remains attractive while $S$ always remains replusive so we need not make any modifications. Such

problems do arise in the nucleon-nucleon scattering problem. See [60] for a discussion of these problems and their resolution.

\section{NUMERICAL SPECTRAL RESULTS}

\section{A. Tabulation and Discussion of Computed Meson Spectra}

We now use our formalism as embodied in Eqs. (2.35), and (3.8a,3.8b) to calculate the full meson spectrum including the light-quark mesons. (As a check on these calculations 
we have also used the older forms derived in [25]). Note that the nonrelativistic quark model when used in conjunction with realistic QCD potentials such as Richardson's potential or the Adler-Piran potential fails for light mesons since the ordinary nonrelativistic Schrödinger equation's lack of relativistic kinematics leads to increasing meson masses as the quark masses drop below a certain point [64], thereby spoiling proper treatment of the pion, as well as other states. Here, we shall see how our relativistic equations remedy this situation. In addition to including the proper relativistic kinematics, our equations also contain energy dependence in the dynamical quasipotential. Mathematically, this feature turns our equations into wave equations that depend nonlinearly on the eigenvalue. Their solution, which we have treated in detail elsewhere (see $[34,61]$ ), requires an efficient iteration scheme to supplement our algorithm for the eigenvalue $b^{2}(w)$ when our equations are written as coupled Schrödinger-like forms.

We display our results in Table I at the end of the paper. In the first two columns of each of the tables we list quantum numbers and experimental rest mass values for 89 known mesons. We include all well known and plausible candidates listed in the standard reference ([57]). We omit only those mesons with substantial flavor mixing. In the tables, the quantum numbers listed are those of the $\phi_{+}$part of the sixteen-component wave function. To generate the fits, in addition to the the quark masses we employ the parameters $\Lambda, U_{0}$ and $\beta$. We merely insert the static Adler-Piran potential into our relativistic wave equations just as we have inserted the Coulomb potential $\mathcal{A}=-\alpha / r$ to obtain the results of $\mathrm{QED}[25,35]$. Note especially that we use a single $\Phi(\mathcal{A}, S)$ for all quark mass ratios - hence a single structure for all the $\bar{Q} Q, \bar{Q} q$, and $\bar{q} q$ mesons in a single overall fit. In the third column in Table I we present the results for the model defined by Eqs.(3.8a,3.8b). The entire confining part of the potential in this model transforms as a world scalar. In our equations, this structure leads to linear confinement at long distances and quadratic confinement at extremely long distances (where the quadratic contribution $S^{2}$ outweighs the linear term $2 m_{w} S$ ). At distances at which $\exp (-\beta \Lambda r)<<1$, the corresponding spin-orbit, Thomas, and Darwin terms are dominated by the scalar interaction, while at short distances $(\exp (-\beta \Lambda r) \sim 1)$ the electromagnetic-like portion of the interaction gives the dominant contribution to the fine structure. Furthermore because the signs of each of the spin-orbit and Darwin terms in the Pauli-form of our Dirac equations are opposite for the scalar and vector interactions, the spin-orbit contributions of those parts of the interaction produce opposite effects with degrees of cancellation depending 
on the size of the quarkonium atom.

We obtain the meson masses given in column three as the result of a least squares fit using the known experimental errors, an assumed calculational error of $1.0 \mathrm{MeV}$, and an independent error conservatively taken to be $5 \%$ of the total width of the meson. We employ the calculational error not to represent the uncertainty of our algorithm but instead to prevent the mesons that are stable with respect to the strong interaction from being weighted too heavily. Our $\chi^{2}$ is per datum (89) minus parameters (8).

The resulting best fit turns out to have quark masses $m_{b}=4.877, m_{c}=1.507, m_{s}=$ $0.253, m_{u}=0.0547, m_{d}=.0580 \mathrm{GeV}$, along with potential parameters $\Lambda=0.216, \Lambda U_{0}=$ $1.865 \mathrm{GeV}$ and inverse distance parameter $\beta=1.936$. This value of $\beta$ implies that (in the best fit) as the quark separation increases, our apportioned Adler-Piran potential switches from primarily vector to scalar at about 0.5 fermi. This shift is a relativistic effect since the effective nonrelativistic limit of the potential $(\mathcal{A}+S)$ exhibits no such shift (i.e., by construction $\beta$ drops out).

In Table I, the numbers given in parentheses to the right of the experimental meson masses are experimental errors in $\mathrm{MeV}$. The numbers given in parentheses to the right of the predicted meson masses are the contribution of that meson's calculation to the total $\chi^{2}$ of 101 .

The 17 mesons that contain a $b$ (or $\bar{b}$ ) quark contribute a total of about 5.4 to the $\chi^{2}$, at an average of about 0.3 each. This is the lowest contribution of those given by any family. Since the Adler-Piran potential was originally derived for static quarks, one should not be surprised to find that most of the best fit mesons are members of the least relativistic of the meson families. Note, however, that five of the best fit mesons of this type contain highly relativistic $u$ and $s$ quarks (for which our equation reduces essentially to the one-body Dirac equation for the light quark).

The 24 mesons that contain a $c$ (or $\bar{c}$ ) quark contribute a total of about 50.7 to the $\chi^{2}$ at an average of about 2.2 each. This is the highest contribution of those given by any family. A significant part of this contribution is due to the $\psi$ meson mass being about $32 \mathrm{MeV}$ off its experimental value. Another part of the contribution is due to fact that the mass of the high orbital excitation of the $D^{*}$ tensor meson is $80 \mathrm{MeV}$ below its experimental value. In addition, the high orbital excitation of the $D_{s}^{*}$ is $60 \mathrm{MeV}$ low.

The 24 mesons that contain an $s$ (or $\bar{s}$ ) quark contribute a total of about 46.3 to the $\chi^{2}$ 
at an average of about 1.3 each, less than that for the $c$-quark mesons. This is important because the $s$ quarks are lighter than the $c$ quarks. Part of the reason for this unexpected effect is that our $\chi^{2}$ fitting procedure accounts for the fact that our meson model ignores the level shifts (due to the instability of many of the mesons that contain an $s$ quark) through the introduction of a theoretical error on the order of $5 \%$ of the width of the unstable mesons.

The 36 mesons that contain a $u$ (or $\bar{u}$ ) quark contribute a total of about 54.6 to the $\chi^{2}$ at an average of about 1.5 each while the 16 mesons on our list that contain a $d$ (or $\bar{d}$ ) quark contribute a total of about 18.6 to the $\chi^{2}$ at an average of about 1.2 each.

The worst fits produced by our model are those to the $\psi$ and the $D^{*}$ and $D_{s}^{*}$ high orbital excitations. Although two of these mesons contain the light $u$ and $d$ quarks, in our fit the more relativistic bound states are not in general fit less well. In fact, the $\pi, K, D$ and $\rho$ mesons are fit better than these two excited $D^{*}$ and $D_{s}^{*}$ mesons.

We see that over all, the two-body Dirac equations together with the relativistic version of the Adler-Piran potential account very well for the meson spectrum over the entire range of relativistic motions, using just the two parametric functions $\mathcal{A}$ and $S$.

We now examine another important feature of our method: the goodness with which our equations account for spin-dependent effects (both fine- and hyperfine- splittings). Table I shows that the best fit versus experimental, ground state singlet-triplet splittings for the $b \bar{u}, b \bar{s}, c \bar{c}, c \bar{u}, c \bar{d}, c \bar{s}, s \bar{u}, s \bar{d}, u \bar{d}$ systems are 48 vs 46, 59 vs 47, 151 vs 117, 134 vs 142, 132 vs 142,147 vs 144, 418 vs 398, 418 vs 398, and finally 648 vs $627 \mathrm{MeV}$. We obtain a uniformly good fit for all hyperfine ground state splittings except for the $\eta_{c}-\psi$ system. The problem with the fit for that system of mesons occurs because the $D^{*}{ }^{3} P_{2}$ and $D_{s}^{*}{ }^{3} P_{2}$ states are significantly low while the $\psi$ is significantly high. Furthermore, the singlet and triplet $P$ states are uniformly low. An attempt to lower the $c$ quark mass by correcting the $\psi$ mass while raising the charmonium and $D^{*}, D_{s}^{*} P$ state masses would require raising the $c$ quark mass. Reducing one discrepancy would worsen the other. Below, we will uncover what we believe is the primary cause for this discrepancy as we examine other aspects of the spectrum.

For the spin-orbit splittings we obtain values for the $R$ ratios $\left.\left({ }^{3} P_{2}-{ }^{3} P_{1}\right) /\left({ }^{3} P_{1}-{ }^{3} P_{0}\right)\right)$ of $0.71,0.67,0.42,-0.19,-0.58,-3.35$ for the two $b \bar{b}$ triplets, and the $c \bar{c}, s \bar{s}, u \bar{s}, u \bar{d}$ spin triplets compared to the experimental ratios of $0.66,0.61,0.48,0.09,-0.97,-0.4$. This fit ranges from very good in the case of the light $\Upsilon$ multiplet to miserably bad for the two lightest multiplets. 
From the experimental point of view some of the problem may be blamed on the uncertain status of the ${ }^{3} P_{0}$ light quark meson bound states and the spin-mixing in the case of the $K^{*}$ multiplet. From the theoretical point of view, the lack of any mechanism in our model to account for the effects of decay rates on level shifts undoubtedly has an effect. Another likely cause is that as we proceed from the heavy mesons to the light ones, the radial size of the meson grows so that the long distance interactions, in which the scalar interaction becomes dominant, play a more important role. The spin-orbit terms due to scalar interactions are opposite in sign to and tend (at long distance) to dominate the spin-orbit terms due to vector interactions. This results in partial to full multiplet inversions as we proceed from the $s \bar{s}$ to the $u \bar{d}$ mesons. This inversion mechanism may also be responsible for the problems of the two orbitally excited $D^{*}$ and $D_{s}^{*}$ mesons described above. It may be responsible as well for the problem of the singlet and triplet $P$ states since the scalar interaction tends to offset the dominant shorter range vector interaction, at least slightly.

We also examine the effect of the hyperfine structure of our equations on the splitting between the ${ }^{1} P_{1}$ and weighted sum $\left[5\left({ }^{3} P_{2}\right)+3\left({ }^{3} P_{1}\right)+1\left({ }^{3} P_{0}\right)\right] / 9$ of bound states. We obtain pairs of values equal to $3.520,3.520 ; 1.408,1.432 ; 1.392,1416$ for the $c \bar{c}, u \bar{s}, u \bar{d}$ families versus the experimental pairs of 3.526,3.525;1.402,1.375;1.231,1.303. The agreement of the theoretical and experimental mass differences is excellent for the $\psi$ system, slightly too large and of the wrong sign for the $K$ system and too small and of the wrong sign for the $u \bar{d}$ system. Part of the cause of this pattern is that pure scalar confinement worsens the fit for the light mesons because of its tendency to reverse the spin-orbit splitting, thereby shifting the center of gravity. The agreement, however, for the light systems is nevertheless considerably better than that in the case of the fine structure splitting $R$ ratios. Another part of the discrepancy may be due to the uncertain status of the light ${ }^{3} P_{0}$ meson as well as the spin-mixing in the case of the $K^{*}$ multiplet. Note that in the case of unequal mass $P$ states, our calculations of the two values incorporate the effects of the $\vec{L} \cdot\left(\vec{s}_{1}-\vec{s}_{2}\right)$ spin-mixing effects. (The use of nonrelativistic notation is only for convenience.)

These differences between heavy and light meson systems also occur in the mixing due to the tensor term between radial $S$ and orbital $D$ excitations of the spin-triplet ground states. This mixing occurs most notably in the $c \bar{c}, u \bar{s}$ and $u \bar{d}$ systems. The three pairs of values that we obtain are 3.808,3.688;1.985,1.800;1.986,1.775 respectively versus the data $3.770,3.686 ; 1.714,1.412 ; 1.700 ; 1.450$. Our results are quite reasonable for the charmonium 
system but underestimate considerably the splitting for the light quark systems. As happened for the significant disagreement in the case of the fine structure, our results here worsen significantly for the light meson systems. The spectroscopy of the lighter mesons is undoubtedly more complex due to their extreme instability (not accounted for in our approach). Note, however, that for the spin-spin hyperfine splittings of the ground states the more relativistic (lighter quark) systems yield masses that agree at least as well with the experimental data as do the heavier systems. This same mixed behavior shows up again for the radial excitations.

The incremental $\chi^{2}$ contributions for the six ${ }^{3} S_{1}$ states of the $\Upsilon$ system is just 1.8. It is 12.9 over three states for the triplet charmonium system (primarily due to the $\psi$ deviation), 3.0 for the two $\phi$ states, 1.6 for the three ${ }^{1} S_{0}$ states of the $K$ system (note, however that these fits include expected errors due to the lack of level shift mechanisms and are thus reduced), 7.3 for the two ${ }^{3} S_{1}$ states of for the $K^{*}$ system, 2.2 for the three triplet $u \bar{d}$ states and 8.2 for the three singlet $u \bar{d}$ states. The $\chi^{2}$ contribution at first increases, then decreases with the lighter systems. Overall, the masses are much too large for the radially excited light quark mesons. These discrepancies may be due both to neglect of decay-induced level shifts and to the increased confining force for large $r$ from linear to quadratic (there is no term to compensate for the quadratic $S^{2}$ term).

The isospin splitting that we obtain for the spin singlet $B$ meson system is $1 \mathrm{MeV}$. Our calculation includes the contribution from the $u-d$ mass difference of $3.3 \mathrm{MeV}$ as well as that due to different charge states. The effect of the latter tends to offset the effects of the former since the $b$ and $\bar{u}$ have the same sign of the charge while the $b$ and $\bar{d}$ have the opposite while $m_{d}>m_{u}$. In the experimental data this offset is complete (0). In the case of the $D^{+}-D^{0}$ splitting our mass difference of $7 \mathrm{MeV}$ represents the combined effects of the $u-d$ mass difference and the slightly increased electromagnetic binding present in the case of the $D^{0}$ and the slightly decreased binding in the case of the $D^{+}$. The experimental mass difference is just $4 \mathrm{MeV}$. These effects work in the same way for the spin-triplet splitting resulting in the theoretical value $5 \mathrm{MeV}$ compared with the experimental value $3 \mathrm{MeV}$. For the ${ }^{3} P_{2}$ isodoublet we obtain $4 \mathrm{MeV}$ versus about 0 for the experimental values. Our isospin splittings are enhanced because of the large $u-d$ quark mass difference that gives the best overall fit. For the $K-K^{*}$ family the experimental value for the isospin splitting is $4 \mathrm{MeV}$ for the singlet and triplet ground states. This splitting actually grows for the 
orbital excitation $\left(K_{2}^{*}\right)$ to $7 \mathrm{MeV}$. The probable reason for this increase is that at the larger distances, the weak influence of the Coulomb differences becomes small while only the actual $u-d$ mass difference influences the result (although it does seem rather large). It is difficult to understand why our results stay virtually zero for all three isodoublets. Note that as with the $B$ doublets, the theoretical contributions of the combined effects of the $u-d$ mass differences and the electrostatic effects tend to cancel. However, the experimental masses do not show this expected cancellation.

\section{Implications of our Model for the New 2.32 GeV Ds Meson}

Recently, the BaBar Collaboration [58] found evidence for a new $0^{+}$strange-charmed meson at $2.32 \mathrm{GeV}$. Using the parameters above and assuming the state is a ${ }^{3} P_{0} c \bar{s}$ meson we find a predicted mass of $2.35 \mathrm{GeV}$, about $130 \mathrm{MeV}$ below our predicted value for the ${ }^{3} P_{2}$ counterpart. The corresponding mass difference in the Godfrey-Isgur model is 2.590-2.480 $=110 \mathrm{MeV}$. Both are well off the experimental mark of $2.572-2.317=255 \mathrm{MeV}$. It is not surprising that its place in the quark model has been the subject of some debate.

Overall comparison with the experimental data shows that the primary strength of our approach is that it provides very good estimates for the ground states for all families of mesons and for the radial excitation and fine structure splittings for the heavier mesons. On the other hand, it overestimates the radial and orbital excitations for the light mesons. Its worst results are those for the fine structure splittings for the $u \bar{s}, d \bar{s}$ and $u \bar{d}$ mesons. Both weaknesses are probably due to long distance scalar potential effects. Below, we shall discuss other aspects of our fit to the spectrum when we compare its results to those of other approaches to the relativistic two-body bound state problem.

\section{B. Explicit Numerical Construction of Meson Wave Functions}

There are 89 mesons in our fit to the meson spectrum. An important advantage of the constraint formalism is that its local wave equation provides us with a direct way to picture the wave functions. As examples, we present the wave functions that result from our overall spectral fit for three mesons: the $\pi$ (Figure 1), for which we present the radial part of $\phi_{+}=\psi_{1}+\psi_{4}$ that solves Eq.(A.5); 


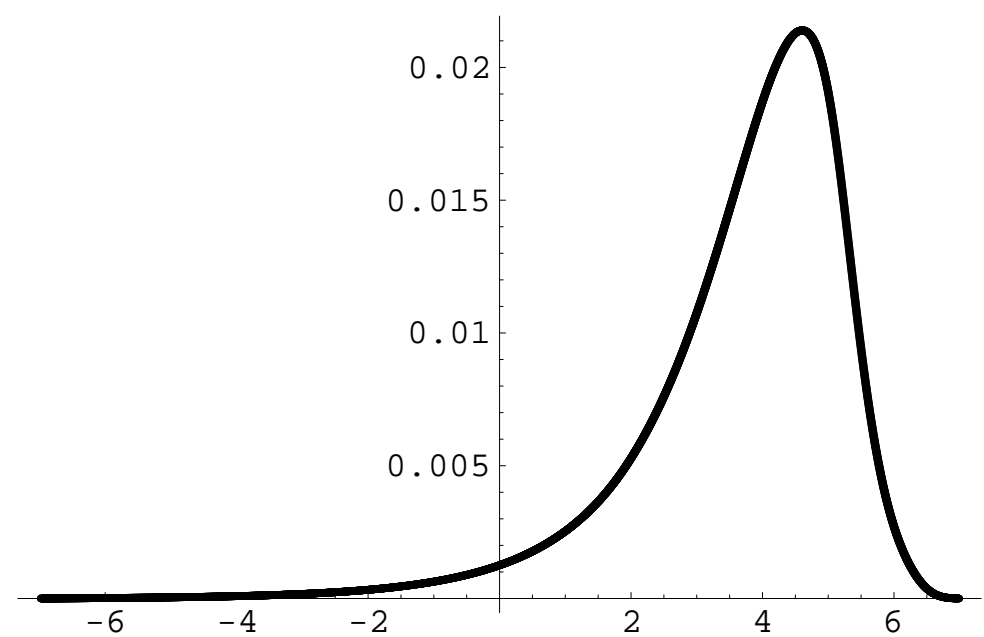

FIG. 1: The $\pi$ wave function plotted against $x=\log \left(r / r_{0}\right)$

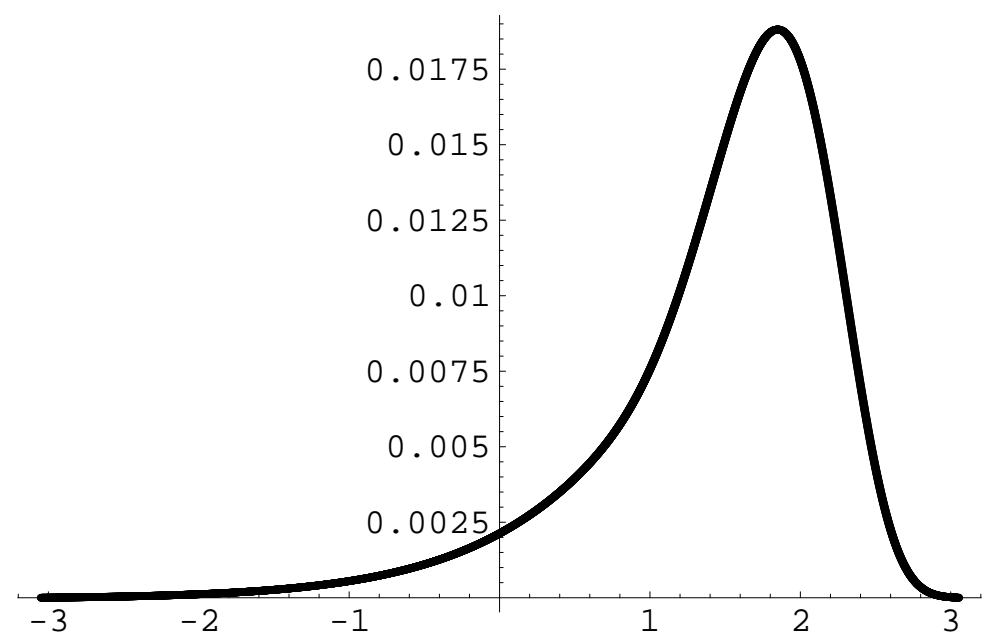

FIG. 2: The $\rho(S)$ wave functions plotted against $x=\log \left(r / r_{0}\right)$

the $\rho$ (Figures 2 and 3) for which we present the radial parts of the wave functions $\phi_{+}$ for both $S$ and $D$ states that solve Eqs.(A.11,A.17) ; and the $\psi / J$ (Figures 4 and 5) for which we present the radial parts of the wave functions $\phi_{+}$for both $S$ and $D$ states that also solve Eq.(A.11,A.17). In each plot the scale $r_{0}$ is proportional to the Compton wavelength corresponding to the nonrelativistic reduced mass $\mu$ of the two quark system. In the table below, for each of the plotted mesons, we give the scale factor $r_{0}$ and the root mean square radius (in Fermis) computed from these meson wave functions. For the $\rho$ and $\psi$ mesons we 


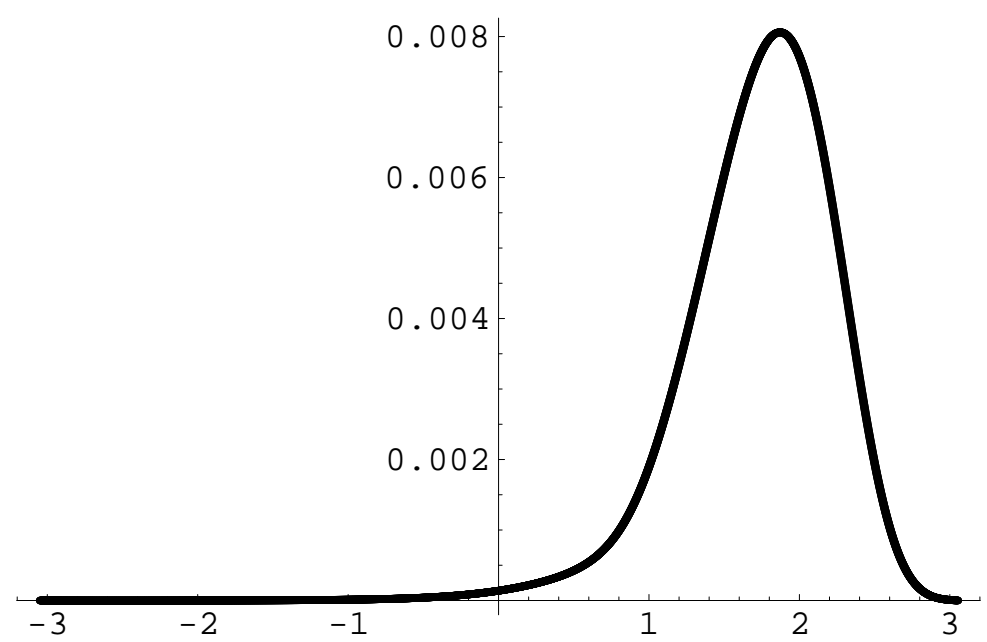

FIG. 3: The $\rho(D)$ wave function plotted against $x=\log \left(r / r_{0}\right)$

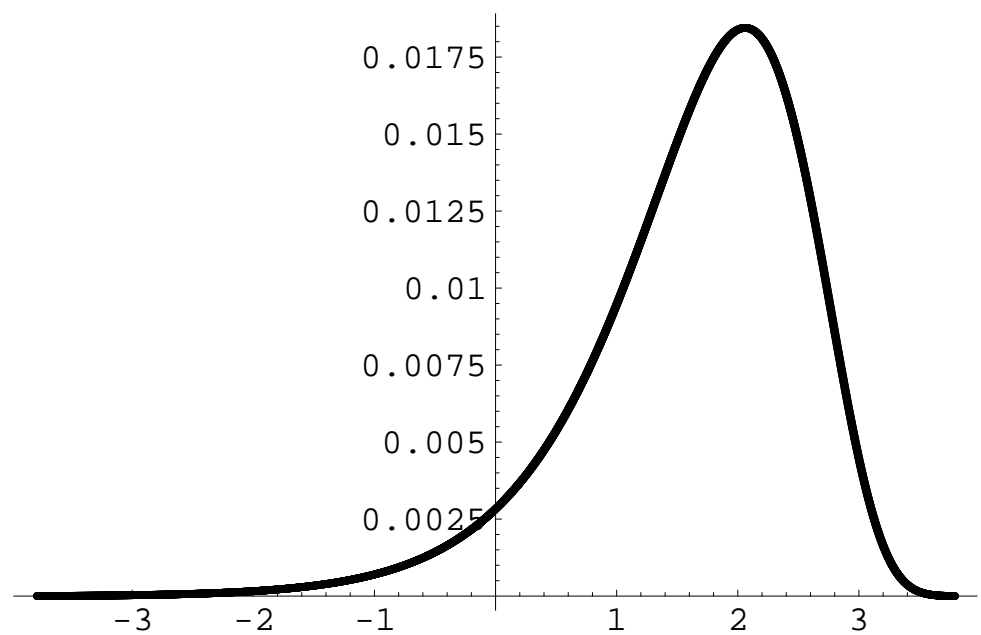

FIG. 4: The $\psi(S)$ wave function plotted against $x=\log \left(r / r_{0}\right)$

also give the computed probabilities for residing in the $S$ and $D$ states.

$$
\begin{array}{lllll}
\text { Meson } & r_{0} \mu & \sqrt{<r^{2}>} S & D \\
\pi & 0.0004 & 0.21 \mathrm{fm} & 1.00 & 0.0 \\
\rho & 0.013 & 0.73 \mathrm{fm} & 0.861 & 0.139 \\
\psi & 0.084 & 0.36 \mathrm{fm} & 0.9974 & 0.0026
\end{array}
$$

Using a scheme outlined in Appendix B, we obtain an analytic approximation to the meson wave functions in terms of harmonic oscillator wave functions. The two primary parameters we use for each meson are the scale factor $a$ and the leading power (short 


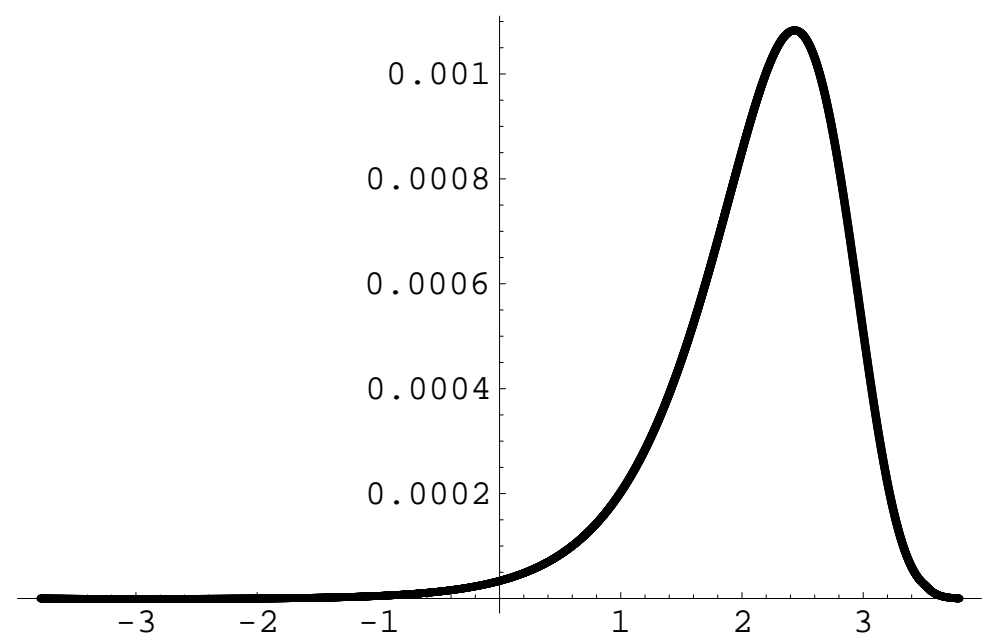

FIG. 5: The $\psi(D)$ wave function plotted against $x=\log \left(r / r_{0}\right)$

distance behavior) exponent $k$. In addition we take as parameters the coefficients of the associated Laguerre polynomials. We write the radial wave function for each meson in the form

$$
u(r) \doteq \sum_{n=0}^{N} c_{n} v_{n}(r)
$$

where

$$
v_{n}(r)=\sqrt{\frac{2(n !)}{(n+k-1 / 2) !}} \exp \left(-y^{2} / 2\right) y^{k} L_{n}^{k-1 / 2}\left(y^{2}\right)
$$

in which $y=r / a=\alpha e^{x}$ and (with $z=y^{2}$ )

$$
L_{n}^{k-1 / 2}(z)=\frac{e^{z} z^{-k+1 / 2}}{n !} \frac{d^{n}}{d z^{n}}\left(e^{-z} z^{k+n-1 / 2}\right)
$$

We then vary the two parameters $a$ and $k$ to obtain the best fit. The coefficients are fixed by

$$
c_{n}=\int_{0}^{+\infty} v_{n}(r) u(r) d r
$$

For meson radial wave functions with more than one component (like the $\psi / J$ ) we fit each component separately. In the table below we give a typical list for parameters $a, k, c_{n}$ for the $\pi, \rho$, and $\psi / J$. 


\begin{tabular}{|c|c|c|c|}
\hline & $\pi$ & $\rho$ & $\psi / J$ \\
\hline$k$ & $2.30734 \mathrm{E}-001$ & $9.85790 \mathrm{E}-001$ & $9.27248 \mathrm{E}-001$ \\
\hline$\alpha$ & $1.22106 \mathrm{E}-004$ & $2.04708 \mathrm{E}-001$ & $5.85947 \mathrm{E}-002$ \\
\hline$c_{0}$ & $-9.70613 \mathrm{E}-001$ & $5.68290 \mathrm{E}-001$ & 8.63401E-001 \\
\hline 1 & $1.97188 \mathrm{E}-001$ & $-5.54267 \mathrm{E}-001$ & $-3.77851 \mathrm{E}-001$ \\
\hline$c_{2}$ & $-1.18926 \mathrm{E}-001$ & 4.55647E-001 & 2.70111E-001 \\
\hline 3 & $3.93232 \mathrm{E}-002$ & $-2.95969 \mathrm{E}-001$ & $-1.44888 \mathrm{E}-001$ \\
\hline$c_{4}$ & $-4.74935 \mathrm{E}-002$ & $2.11945 \mathrm{E}-001$ & $1.05621 \mathrm{E}-001$ \\
\hline$c_{5}$ & $1.59519 \mathrm{E}-002$ & $-1.29901 \mathrm{E}-001$ & $-5.85549 \mathrm{E}-002$ \\
\hline$c_{6}$ & $-2.21638 \mathrm{E}-002$ & $8.87707 \mathrm{E}-002$ & $4.46522 \mathrm{E}-002$ \\
\hline$c_{7}$ & $9.35388 \mathrm{E}-003$ & $-5.36537 \mathrm{E}-002$ & $-2.44101 \mathrm{E}-002$ \\
\hline$c_{8}$ & $-1.12997 \mathrm{E}-002$ & $3.57731 \mathrm{E}-002$ & $1.98781 \mathrm{E}-002$ \\
\hline$c_{9}$ & $5.74799 \mathrm{E}-003$ & $-2.16185 \mathrm{E}-002$ & $-1.03167 \mathrm{E}-002$ \\
\hline$c_{10}$ & $-6.24195 \mathrm{E}-003$ & $1.42167 \mathrm{E}-002$ & $9.24913 \mathrm{E}-003$ \\
\hline$c_{11}$ & $3.44862 \mathrm{E}-003$ & $-8.57381 \mathrm{E}-003$ & $-4.34130 \mathrm{E}-003$ \\
\hline$c_{12}$ & $-3.63673 \mathrm{E}-003$ & $5.67698 \mathrm{E}-003$ & $4.49675 \mathrm{E}-003$ \\
\hline$c_{13}$ & $2.04307 \mathrm{E}-003$ & $-3.31349 \mathrm{E}-003$ & $-1.77086 \mathrm{E}-003$ \\
\hline$c_{14}$ & $-2.16019 \mathrm{E}-003$ & $2.33901 \mathrm{E}-003$ & $2.29266 \mathrm{E}-003$ \\
\hline$c_{15}$ & $1.22870 \mathrm{E}-003$ & $-1.19431 \mathrm{E}-003$ & $-6.63516 \mathrm{E}-004$ \\
\hline$c_{16}$ & $-1.26919 \mathrm{E}-003$ & $1.03806 \mathrm{E}-003$ & $1.23170 \mathrm{E}-003$ \\
\hline$c_{17}$ & $7.72030 \mathrm{E}-004$ & $-3.42741 \mathrm{E}-004$ & $-1.93158 \mathrm{E}-004$ \\
\hline$c_{18}$ & $-7.16255 \mathrm{E}-004$ & $5.20857 \mathrm{E}-004$ & $6.97788 \mathrm{E}-004$ \\
\hline$c_{19}$ & $5.18700 \mathrm{E}-004$ & $-5.02603 \mathrm{E}-006$ & $-3.64677 \mathrm{E}-007$ \\
\hline$c_{2}$ & $-3.71156 \mathrm{E}-004$ & & \\
\hline b & $3.77233 \mathrm{E}-004$ & & \\
\hline & & & \\
\hline
\end{tabular}

We note several features. First, the fit to the $\pi$ wave function appears to converge significantly more slowly than those for the $\rho$ and $\psi / J$. (We do not present plots comparing the numerical wave function with the harmonic oscillator wave function fits since there are no visible differences). Also note that the $\pi$ 's short distance behavior is distinctly different from those of the other two, having a stronger radial dependence at the origin. All three 
wave functions possess polynomial coefficients that exhibit an oscillatory behavior.

\section{Numerical Evidence for Goldstone Boson Behavior}

In our equations, the pion is a Goldstone boson in the sense that its mass tends toward zero numerically in the limit in which the quark mass numerically goes toward zero. This may be seen in the accompanying plot Figure 6 (units are in $\mathrm{MeV}$ ). Note that the $\rho$ meson mass approaches a finite value in the chiral limit. This non-Goldstone behavior also holds for the excited pion states. None of the alternative approaches discussed in the following sections have displayed this property. Another distinction we point out is that our $u$ and $d$ quark masses (on the order of 55-60 MeV) are significantly smaller than the constituent quark masses appearing in most all other models (on the order of $300 \mathrm{MeV}$ ) - closer to the small current quark masses of a few $\mathrm{MeV}$. Note, however, that the shape of our pion curve is not what one would expect from the Goldberger-Trieman relation

$$
m_{q}=m_{\pi}^{2} F_{\pi}
$$

Thus this aspect of our model requires further investigation.

\section{COMPARISON OF STRUCTURES OF TWO-BODY DIRAC EQUATIONS WITH THOSE OF ALTERNATIVE APPROACHES}

So far, we have obtained spectral results given by our equations when solved in their own most convenient form. In Sections (VI-IX) we shall compare our results with recent universal fits to the meson spectrum produced by a number of other authors. These approaches employ equations whose structures (at first sight) appear radically different from ours. However,

as we have shown elsewhere [35], because our approach starts from a pair of coupled but compatible Dirac equations, these equations can be rearranged in a multitude of forms all possessing the same solutions. Among the rearrangements are those with structures close to those of the authors whose spectral fits we shall shortly examine. In order to see how structural differences in each case may lead to differences in the resulting numerical spectra, we shall begin by considering relevant rearrangements of the two-body Dirac equations.

The first two alternative approaches which we shall discuss use truncated versions of the Bethe-Salpeter equation (Salpeter and quasipotential) while the third uses a modified form 


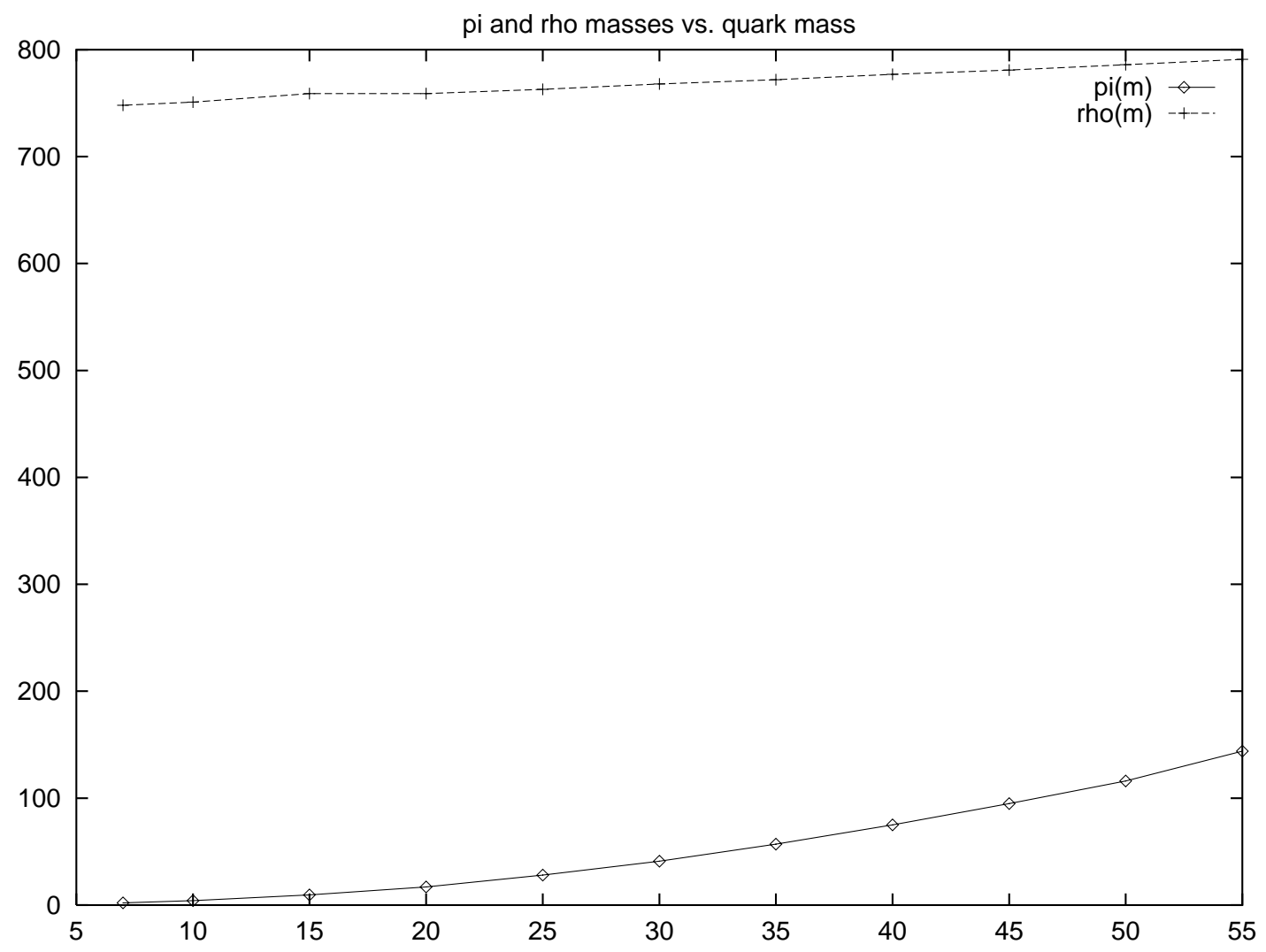

FIG. 6: $\pi$ and $\rho$ masses versus quark mass in $\mathrm{MeV}$

of the Breit equation. In order to relate the detailed predictions of our approach to these alternatives, we need to relate our minimal substitution method for the introduction of interactions to the introduction of interaction through the use of kernels that dominates the older approaches. The field-theoretic kernel employs a direct product of gamma matrices times some function of the relative momentum or coordinate. What is the analog of the kernel in our approach? In earlier work we found that we could obtain our "external potential" or "minimal interaction" form of our two-body Dirac equations from yet another form displaying a remarkable hyperbolic structure. We were able to recast our compatible Dirac 
equations $(2.1 \mathrm{a}, 2.1 \mathrm{~b})$ as

$$
\begin{aligned}
& \mathcal{S}_{1} \psi=\left(\cosh (\Delta) \mathbf{S}_{1}+\sinh (\Delta) \mathbf{S}_{2}\right) \psi=0 \\
& \mathcal{S}_{2} \psi=\left(\cosh (\Delta) \mathbf{S}_{2}+\sinh (\Delta) \mathbf{S}_{1}\right) \psi=0
\end{aligned}
$$

in which $[62]$

$$
\begin{aligned}
& \mathbf{S}_{1} \psi \equiv\left(\mathcal{S}_{10} \cosh (\Delta)+\mathcal{S}_{20} \sinh (\Delta)\right) \psi=0 \\
& \mathbf{S}_{2} \psi \equiv\left(\mathcal{S}_{20} \cosh (\Delta)+\mathcal{S}_{10} \sinh (\Delta)\right) \psi=0
\end{aligned}
$$

in tems of free Dirac operators

$$
\begin{aligned}
& \mathcal{S}_{10} \psi=\left(-\beta_{1} \Sigma_{1} \cdot p+\epsilon_{1} \beta_{1} \gamma_{51}+m_{1} \gamma_{51}\right) \psi \\
& \mathcal{S}_{20} \psi=\left(\beta_{2} \Sigma_{2} \cdot p+\epsilon_{2} \beta_{2} \gamma_{52}+m_{2} \gamma_{52}\right) \psi
\end{aligned}
$$

and the kernel

$$
\Delta=\frac{1}{2} \gamma_{51} \gamma_{52}\left[L\left(x_{\perp}\right)+\gamma_{1} \cdot \gamma_{2} \mathcal{G}\left(x_{\perp}\right)\right]
$$

We then recover the explicit "external potential" forms of our equations, (2.1a,2.1b) from $(5.1,5.2)$ by moving the free Dirac operators $\mathcal{S}_{i 0}$ to the right to operate on the wave function. This rearrangement produces the derivative recoil terms apparent in Eqs.(2.1a,2.1ba)). $\Delta$ may take any one of (or combination of) eight invariant forms. In terms of

$$
\mathcal{O}_{1}=-\gamma_{51} \gamma_{52}
$$

these become $\Delta\left(x_{\perp}\right)=-L\left(x_{\perp}\right) \mathcal{O}_{1} / 2, \gamma_{1} \cdot \hat{P} \gamma_{2} \cdot \hat{P} J\left(x_{\perp}\right) \mathcal{O}_{1} / 2, \gamma_{1 \perp} \cdot \gamma_{2 \perp} \mathcal{G}\left(x_{\perp}\right) \mathcal{O}_{1} / 2$ or $\alpha_{1}$. $\alpha_{2} \mathcal{F}\left(x_{\perp}\right) \mathcal{O}_{1} / 2$ for scalar, time-like vector, space-like vector, or tensor (polar) interactions respectively. Note that in our $\Delta\left(x_{\perp}\right)$ in Eq.(5.4) above, $\mathcal{G}\left(x_{\perp}\right)$ enters multiplied by the electromagnetic-like combination $\gamma_{1} \cdot \gamma_{2}=-\gamma_{1} \cdot \hat{P} \gamma_{2} \cdot \hat{P}+\gamma_{1 \perp} \cdot \gamma_{2 \perp}$ of time and space-like parts. This structure appears as a result of our use of the Lorentz gauge to introduce vector interactions in the classical version of the constraint equations or as a result of our use of the Feynman gauge to treat the field-theoretic version[55]. The axial counterparts to the constraints with polar interactions are given by (note the minus sign compared with the plus sign in Eqs.(5.1)) [62]

$$
\begin{aligned}
& \mathcal{S}_{1} \psi=\left(\cosh (\Delta) \mathbf{S}_{1}-\sinh (\Delta) \mathbf{S}_{2}\right) \psi=0 \\
& \mathcal{S}_{2} \psi=\left(\cosh (\Delta) \mathbf{S}_{2}-\sinh (\Delta) \mathbf{S}_{1}\right) \psi=0
\end{aligned}
$$


in which $\mathbf{S}_{1}$ and $\mathbf{S}_{2}$ are still given by (5.2) with axial counterparts to the above $\Delta$ 's given by $C\left(x_{\perp}\right) / 2, \gamma_{51} \gamma_{1} \cdot \hat{P} \gamma_{52} \gamma_{2} \cdot \hat{P} H\left(x_{\perp}\right) \mathcal{O}_{1} / 2, \gamma_{51} \gamma_{1 \perp} \cdot \gamma_{52} \gamma_{2 \perp} I\left(x_{\perp}\right) \mathcal{O}_{1} / 2$ and $\sigma_{1} \cdot \sigma_{2} Y\left(x_{\perp}\right) \mathcal{O}_{1} / 2$ respectively. The advantage of the hyperbolic form is that with its aid we may first choose among the 8 interaction types in an unambiguous way to introduce interaction (without struggling to restore compatibility) and then, for computational convenience, transform the Dirac equations to "external potential" form. In the weak-potential limit of our equations, the coefficients of $\gamma_{51} \gamma_{52}$ in the expansion of our $\Delta$ interaction matrix in Eq.(5.4) directly correspond to the interaction kernels of the Bethe-Salpeter equation. Note however, that because of the hyperbolic structure, what we call a "vector interaction" actually corresponds to a particular combination of vector and pseudovector interactions in the older approaches (see Eq.(5.15) below).

This difference in classification of interactions becomes apparent when we put our equations into a Breit-like form. Consider the linear combination

$$
\beta_{1} \gamma_{51} \mathbf{S}_{1}+\beta_{2} \gamma_{52} \mathbf{S}_{2}
$$

For later convenience, form the interaction matrix

$$
\mathcal{D}\left(x_{\perp}\right)=\frac{1}{2} \beta_{1} \gamma_{51} \beta_{2} \gamma_{52} \Delta\left(x_{\perp}\right)
$$

After simplification, the linear combination Eq.(5.7) of our two hyperbolic equations becomes

$$
w \Psi=\left[H_{10}+H_{20}+V\left(x_{\perp}, \alpha_{1}, \alpha_{2}, \beta_{1}, \beta_{2}, \gamma_{51}, \gamma_{52}\right)\right] \Psi
$$

in which

$$
\Psi=\exp (-\mathcal{D}) \psi
$$

and

$$
H_{10}=\alpha_{1} \cdot p_{\perp}+\beta_{1} m_{1}, H_{20}=-\alpha_{2} \cdot p_{\perp}+\beta_{2} m_{2}
$$

For the electromagnetic vector kernel $\Delta\left(x_{\perp}\right)=\frac{1}{2}\left[\gamma_{51} \gamma_{52}\right] \gamma_{1} \cdot \gamma_{2} \mathcal{G}\left(x_{\perp}\right), \mathcal{D}$ then becomes

$$
\mathcal{D}=\frac{1}{2} \mathcal{G}\left(x_{\perp}\right)\left(\alpha_{1} \cdot \alpha_{2}-1\right)
$$

so that the relativistic Breit-like equation takes the c.m. form

$$
w \Psi=\left[\boldsymbol{\alpha}_{1} \cdot \mathbf{p}-\boldsymbol{\alpha}_{2} \cdot \mathbf{p}+\beta_{1} m_{1}+\beta_{2} m_{2}+w\left(1-\exp \left[\mathcal{G}(\mathbf{r})\left(\boldsymbol{\alpha}_{1} \cdot \boldsymbol{\alpha}_{2}-1\right)\right]\right)\right] \Psi
$$


In lowest order this equation takes on the familiar form for four-vector interactions (seemingly missing the traditional Darwin interaction piece

$\left.\sim \hat{\mathbf{r}} \cdot \boldsymbol{\alpha}_{1} \hat{\mathbf{r}} \cdot \boldsymbol{\alpha}_{2}\right)$.

$$
w \Psi=\left[\boldsymbol{\alpha}_{1} \cdot \mathbf{p}-\boldsymbol{\alpha}_{2} \cdot \mathbf{p}+\beta_{1} m_{1}+\beta_{2} m_{2}-w \mathcal{G}(\mathbf{r})\left(\boldsymbol{\alpha}_{1} \cdot \boldsymbol{\alpha}_{2}-1\right)\right] \Psi
$$

However, as we first showed in [45], expanding the simple structure of Eq.(5.13) to higher order in fact generates the correct Darwin dynamics. As a consequence, our unapproximated equation yields analytic and numerical agreement with the field theoretic spectrum through order $\alpha^{4}$. Explicitly, our full interaction is

$$
\begin{aligned}
\exp \left[\left(\boldsymbol{\alpha}_{1} \cdot \boldsymbol{\alpha}_{2}-1\right) \mathcal{G}\right] & =\frac{\exp (-\mathcal{G})}{4}\left[3 \cosh (\mathcal{G})+\cosh (3 \mathcal{G})+\gamma_{51} \gamma_{52}(3 \sinh (\mathcal{G})-\sinh (3 \mathcal{G}))\right. \\
& \left.+\boldsymbol{\alpha}_{1} \cdot \boldsymbol{\alpha}_{2}(\sinh (3 \mathcal{G})+\sinh (\mathcal{G}))+\boldsymbol{\sigma}_{1} \cdot \boldsymbol{\sigma}_{2}(\cosh (\mathcal{G})-\sinh (3 \mathcal{G}))\right]
\end{aligned}
$$

so that our Breit-like potential contains a combination of "vector" and "pseudovector" interactions originating from the four-vector potentials of the original constraint equations in "external-potential" form. [63]

In this section we have seen how the two-body Dirac equations with field-theoretic interaction structure automatically retain the correct Darwin structure of QED. Such a demonstration should be carried out for each alternative treatment (if possible) in order to check that truncations and numerical procedures have not destroyed its own version of the fieldtheoretic Darwin structure for its treatment of the vector interaction of QED (and associated vector structures in QCD). Explicitly in our own work we find that including all the couplings to smaller components of the wave function is crucial not only for our nonperturbative QED spectral results (see [25]) but also for our good results for $\pi-\rho$ splittings and the Goldstone behavior of the pion as the quark mass tends toward zero. Without those couplings the good results for the positronium splittings and light mesons evaporate. 


\section{THE WISCONSIN MODEL OF GARA, DURAND, DURAND, AND NICK- ISCH}

\section{A. Definition of The Model and Comparison of Structure with Two-Body Dirac} Approach

The authors of reference [48] base their analysis of quark-antiquark bound states on the reduced Salpeter equation containing a mixture of scalar and vector interactions between quarks of the same or different flavors. When rewritten in a notation that aids comparison with our approach, their bound state equation takes the c.m. form

$$
\left[w-\omega_{1}-\omega_{2}\right] \Phi(\mathbf{p})=\Lambda^{+}(\mathbf{p}) \gamma^{0} \int \frac{d^{3} p^{\prime}}{(2 \pi)^{3}}\left[\mathcal{A}\left(\mathbf{p}-\mathbf{p}^{\prime}\right) \gamma_{\mu} \Phi\left(\mathbf{p}^{\prime}\right) \gamma^{\mu}+S\left(\mathbf{p}-\mathbf{p}^{\prime}\right) \Phi\left(\mathbf{p}^{\prime}\right)\right] \gamma^{0} \Lambda^{-}(-\mathbf{p})
$$

in which $\mathcal{A}$ and $S$ are functions that parametrize the electromagnetic-like and scalar interactions, $\Lambda^{ \pm}$are projection operators, $w$ is the c.m. energy, $\omega_{i}=\left(\mathbf{p}^{2}+m_{i}^{2}\right)^{1 / 2}$, while $\Phi$ is a four by four matrix wave function represented in block matrix form as

$$
\Phi=\left[\begin{array}{l}
\phi^{+-} \phi^{++} \\
\phi^{--} \phi^{-+}
\end{array}\right]
$$

They obtain this equation from the full Bethe-Salpeter equation by making an assumption equivalent to using a position-space description in which they calculate the interaction potential with the equal time constraint, neglecting retardation. (These are the usual ad-hoc assumptions that in our approach are automatic consequences (in covariant form) of our two simultaneous, compatible Dirac equations.) These restrictions turn Eq.(6.1) into the standard Salpeter equation. In addition the Wisconsin group employs what we call the "weak potential assumption": $\left(w+\omega_{1}+\omega_{2}\right)>>V$. This assumption turns Eq.(6.1) into the reduced Salpeter equation which, because of the properties of the projection operator, allows the Wisconsin group to perform a Gordon reduction of its equation to obtain a reduced final equation in terms of $\phi^{++}$alone. In our approach we make no such "weak potential assumption" and therefore must deal directly with the fact that our Dirac equations themselves relate components of the sixteen component wave function to each another. Unlike what happens in the reduced Salpeter equation, in our method this coupling leads to potential dependent denominators, a strong potential structure that we found crucial in demonstrating that our formalism yields legitimate relativistic two-body equations. Just as we do, however, the Wisconsin group works in coordinate space where the dynamical potentials are 
local and easy to handle. However, in their method upon Fourier transformation the kinetic factors $\omega_{i}$ then become nonlocal operators. In contrast, the entire dynamical structure of our two-body Dirac equations is local as long as the potentials are local.

The Wisconsin group uses local static potentials that play the role of our Adler-Piran potential:

$$
\begin{aligned}
& \mathcal{A}(r)=-\frac{4}{3} \frac{\alpha_{s}(r)}{r} e^{-\mu^{\prime} r}+\delta\left(-\frac{\beta}{r}+\Lambda r\right)\left(1-e^{-\mu r}\right) \\
& S(r)=(1-\delta)\left(-\frac{\beta}{r}+B r\right)\left(1-e^{-\mu r}\right)+\left(C+C_{1} r+C_{2} r^{2}\right)\left(1-e^{-\mu r}\right) e^{-\mu r}
\end{aligned}
$$

Note that Gara et al introduce a confining electromagnetic-like vector potential proportional to a parameter $\delta$. This differs from our approach in which the (dominant) linear portion of the confinement potential has no electromagnetic part. Like Adler's potential, theirs has a long range $1 / r$ part (the so-called Luscher term). Its short range part is electromagnetic-like just as is ours, and like Adler's is obtained from a renormalization group equation.

They base their analysis on a nonperturbative, numerical solution of the reduced Salpeter equation Eq.(6.1) with interaction Eq.(6.3).

\section{B. Comparison of Wisconsin Fit with that of Two-Body Dirac Equations}

In Table II we include the Wisconsin variable- $\delta$ (vector and scalar confinement) best fit results, and the best fit results our method gives when restricted to the 25 mesons they consider. For uniformity of presentation we give all of the Wisconsin results in terms of absolute masses (rather than the mass differences and averages these authors presented for the spin-orbit triplets). Although Gara et al. did not perform the same $\chi^{2}$ fit that we do, we present (in parentheses) the incremental $\chi^{2}$ contribution for each meson so that we can easily compare the results of the two methods. We also compare their $R$ values and ${ }^{3} P$ avg. to ours directly in the discussion below.

Our results are closer to the experimental results for 16 of the 25 mesons. In detail, their $R$ values for the $\Upsilon$ and $\psi$ families of $0.83,0.78$, and 0.60 are less accurate than two of our values of $0.64,0.68,0.35$ respectively. Their ${ }^{3} P$ averages $\left[5\left({ }^{3} P_{2}\right)+3\left({ }^{3} P_{1}\right)+1\left({ }^{3} P_{0}\right)\right] / 9$ of $9.902,10.262,3.513$ and ours $(9.901,10.264,3.513)$ are essentially the same compared to the experimental results of $9.900,10.273,3.525 \mathrm{MeV}$. Their hyperfine splittings for the two 
charmonium multiplets of 200 and $47 \mathrm{MeV}$ are significantly worse than our fits of 150 and $79 \mathrm{MeV}$. Their hyperfine splittings for the mesons with one $d$ or $s$ quark are 27, 51, and 127 $\mathrm{MeV}$. Our fits of $128,138,420 \mathrm{MeV}$ respectively are much closer to the experimental results of $141,141,398 \mathrm{MeV}$.

The radial excitation energies for the two lowest $\Upsilon$ excitations and the singlet and triplet charmonium excitations are again accounted for significantly better by three of four of our values of $569,335,636,568 \mathrm{MeV}$ for the results in the last column than by the Wisconsin results of $602,331,654,491 \mathrm{MeV}$. In summary, the major strength of our approach is reflected in its better fits to the hyperfine splittings and radial excitations. The Wisconsin group's results for the fine structure splitting are overall about the same as ours. Moreover, even a casual glance at the results shows one glaring discrepancy that results from their approach - their hyperfine splittings for the light quark mesons. The cause of this is probably the fact that their reduced Salpeter approach does not include coupling of the upper-upper piece to the other 12 components of the 16 component wave function. In fact, the lighter the meson, the worse is their result. In our QED numerical investigations we found that couplings to the other components of the wave function were essential in order to obtain agreement with the standard perturbative spectral results of QED. We have found that the same strongpotential effects that led to our successful results in QED are responsible for the goodness of our hyperfine splitting, particularly for the mesons containing the light quarks. It would be important to test the Wisconsin group's procedure (with its deleted couplings to the other wave functions) numerically with $\mathcal{A}=-\alpha / r$ and $S=0$ for positronium to determine whether the problems that the Wisconsin model has with mesonic hyperfine splittings in QCD are reflected in its results for QED.

Gara et al. point out that in their approach the straight line Regge trajectories ( $j$ versus $w^{2}$ ) for the light quark systems are much too steep, with slopes greater than twice the observed slopes for pure scalar confinement. The best fit experimental slope and intercept values for the $\rho, a_{2}, \rho_{3}$ trajectory are $(0.88,0.48)$. The slope and intercept values that we obtain for our model in Table I are $(0.87,0.47)$, in excellent agreement with the best experimental fit. For the $\phi_{1}, f_{2}, \phi_{3}$ trajectory the experimental values are $(0.83,0.11)$ while our model of Table I produces the set of values $(0.85,0.095)$. The intercepts are not as accurate as those for the $\rho$ trajectory although our results actually produce a tighter fit to a straight line trajectory than do the experimental results. Finally we come to the $\pi, b_{1}, \pi_{2}$ trajectory. 
We obtain the values $(0.57,-0.04)$. Compared to the experimental values of $(0.72,-0.04)$ our slopes are about $25-30 \%$ small, although our fit to the straight line is just as tight. The probable reason for the relative advantage of our results over those of the Wisconsin group is that our bound state equations include a strong potential structure, and are not limited by the weak potential approximation built into the reduced Salpeter equation.

\section{THE IOWA STATE MODEL OF SOMMERER, EL-HADY, SPENCE AND VARY}

\section{A. Definition of The Model and Comparison of Structure with Two-Body Dirac} Approach

The Iowa State group introduces a new relativistic quasipotential reduction of the BetheSalpeter equation. They use the well known fact that there are an infinite number of such reductions [65] to construct a formal quasipotential parametrized in terms of two independent constants. They show that when all of the most often used reductions are specialized to QED, they fail to numerically reproduce the correct ground state result for singlet positronium through order $\alpha^{4}[66]$. These authors then fix the free parameters in their quasipotential by requiring that their resulting ground state energy lie close to the well-known perturbative value. In addition, the form of the quasipotential reduction they use produces a projection to positive energy states only. The Iowa State group uses a scalar linear confinement plus massless vector boson exchange-potential with the kernel

$$
\frac{-4 \pi \alpha_{s} \gamma_{0} \gamma_{\mu} \times \gamma_{0} \gamma_{\mu}}{-\left(q-q^{\prime}\right)^{2}}+4 \pi b \lim _{\mu \rightarrow 0}\left[\frac{\partial}{\partial \mu}\right]^{2} \frac{\gamma_{0} \times \gamma_{0}}{-\left(q-q^{\prime}\right)^{2}+\mu^{2}}
$$

The QCD coupling $\alpha_{s}$ that they use is treated as a running coupling constant that depends on the momentum transfer and two parameters. Their quasipotential reduction incorporates zero relative energy in the c.m. frame.

\section{B. Comparison of Fit with that of Constraint Approach}

In Table III, we give the Iowa State group's results for a set of mesons together with our results for the same set of mesons. In the fourth column of this table we present the results we would obtain from our approach if we limited our fit just to the 47 mesons used by the 
Iowa State group. We use the same RMS fitting procedure used by these authors instead of the $\chi^{2}$ fit used in our Table I. The results are quite similar, 50 for the Ohio State model and 53 for our model.

Of the 47 mesons in their table, our fits are closer to the data in 25. Thus, according to this crude measure there is no significant difference between the results of the two approaches. [67] We proceed now with a detailed comparison. Their $R$ values for the two bottomonium and one charmonium multiplets are 3.25,1.09,1.09. Our $R$ values of 0.70,0.74,0.44 are considerably closer to the experimental ratios of $0.66(0.61), 0.56(0.61), 0.47$. (We make no comparison for the three light quark multiplets $(s \bar{s}, s \bar{u}, u \bar{d})$ since the Iowa State Group did not calculate the ${ }^{3} P_{0}$ states. ) We note, however, that for the pairs of $s \bar{u}$ and $u \bar{d}$ their results for ${ }^{3} P_{2}-{ }^{3} P_{1}$ splittings are substantially better than our results. In particular, unlike our results, theirs do not have an inversion of the splitting. Our poor results for these splittings are likely due to a larger influence of the scalar than the vector portion of the spin-orbit interaction. Comparing their ${ }^{3} P$ averages $\left[5\left({ }^{3} P_{2}\right)+3\left({ }^{3} P_{1}\right)+1\left({ }^{3} P_{0}\right)\right] / 9$ of 9.859,3.497,1.433,1.015 GeV for the lowest lying spin-orbit multiplets listed in the table with our values of 9.902,3.516,1.470,1.386 and the experimental results of 9.900,3.527,1.503,1.303 $\mathrm{GeV}$ we see that ours are closer in each case to the experimental results. We see also that for charmonium, our average is nearly equal to our ${ }^{1} P_{1}$ level while the Iowa State results are $75 \mathrm{MeV}$ higher than their ${ }^{1} P_{1}$ level. For the $u \bar{d}$ system, our average is $25 \mathrm{MeV}$ higher than our ${ }^{1} P_{1}$ level while theirs is $122 \mathrm{MeV}$ above their calculated ${ }^{1} P_{1}$ level. Their values of the hyperfine $\left({ }^{3} S_{1}-{ }^{1} S_{0}\right)$ splittings are $98,48,100,108,421,677 \mathrm{MeV}$ for the two charmonium multiplets, and the $D-D^{*}, D_{s}-D_{s}^{*}, K-K^{*}, \pi-\rho$ pairs. Comparison with the experimental splittings of 117,92,142,139,398,628 MeV and our results of 159,82,137,156,376,593 MeV show the constraint results closer to the experimental splittings on all but the ground state charmonium pair. (We have commented earlier on the origin of the descrepancy between our $\psi$ value and the experimental result.) We next wish to compare the results generated in both approaches for the spin-spin effect embodied in the ${ }^{3} P_{1}-{ }^{1} P_{1}$ splittings. For the $c \bar{c}, s \bar{u}, u \bar{d}$ pairs the Iowa State results are 10,43,4 MeV compared to the experimental results of 15,136(129),28 (0) MeV and the two-body Dirac results of 16,78,279 MeV. For the heavier two pairs, the constraint splitting results are substantially closer to the experimental results. This resembles the similar spin-spin pattern found in the $S$-state hyperfine splittings. Our poor result for the $u \bar{d}$ meson has the same origin as our poor result for the 
$R$ value mentioned above. Finally, we compare the radial excitations. The six upsilon states in the experimental column of the table occur at intervals of $562,332,225,285,254 \mathrm{MeV}$. The three charmonium triplet states and the two charmonium singlet states occur at intervals of $589,354,614 \mathrm{MeV}$ while the two $s \bar{s}$ and $u \bar{d}$ states occur at intervals of 661 and $1160 \mathrm{MeV}$. The corresponding Iowa State intervals are 544,335,270,259,226,597,416,647,625,1304 MeV while our intervals are 578,345,260,218,191,560,395,637,753,1331 MeV. The Iowa State radial excitation splittings are closer to the experimental values on four of the five upsilon splittings, one of the three charmonium splittings and both of the lighter quark splittings.

Even though the RMS values obtained in each approach are nearly the same, on most of the detailed comparisons made above the constraint approach appears to give better fits. The exceptions to this are the radial excitations and some of the heavier light-meson excitations. The largest portion of our RMS values come from the heavy-light meson orbital and radial excitations.

We have long argued that any proposed relativistic wave equation should be tested in terms of its ability to reproduce known perturbative results of QED and other relevant relativistic quantum field theories when solved nonperturbatively before being applied to QCD. The Iowa State group in fact adopts this philosophy in order to resolve an ambiguity in the construction of the quasipotential in their wave equation by demanding that it reproduce the ground state level of singlet positronium numerically. This requirement fixes the values of the two parameters of their quasipotential mentioned above. In contrast, the constraint approach has no free parameters of the type used by [49] for the quasipotential reductions. Instead, its Green function is fixed. While within the constraint approach the connection between the kernel and the invariant constraint functions (e.g. $\mathcal{G}, L$ ) does involve some freedom of choice (see Eqs. $(2.14,2.15,2.17)$ ), that freedom is not determined by the requirement that the model fit a particular state but instead is fixed by fundamental dynamical requirements following equivalently from classical or from quantum field theory and resulting in the appearance of a minimal form of the potential (see Eq.(2.21) and below). Several features separate the two approaches. First, as we found in [25] the QED results provided by our equation agree with those of standard perturbative QED for more than just the ground state while it is unknown if the parameters that the Iowa State model uses that ensured its fit to the singlet ground state of positronium would work for the other states. Second the constraint approach generates similar structures for scalar interactions and sys- 
tems of vector and scalar interactions with agreement with the corresponding perturbative field-theoretic results while again it is unknown whether the parameters that the Iowa State model uses that gave good fits to the singlet ground state of positronium would work in the presence of other potentials. Third, the match to singlet positronium that we obtained was an analytic consequence of our equations for QED and therefore a test of those equations [68], not the result of a numerical fit. Fourth, our approach includes essential contributions from all sixteen components of the relativistic wave function, not just the "positive energy" components [69]. Fifth, an important consequence of the fully relativistic dynamics and gauge-theoretic structure of the constraint equations is that they produce values of the light

quark masses closer to current algebra values than do alternative approaches. The quark masses that we obtained in our comparison fit with the Iowa State model are $m_{s}=314$ $\mathrm{MeV}$ and $m_{u}=m_{d}=67 \mathrm{MeV}$ which are significantly closer to the current algebra values of $m_{s} \sim 125 \mathrm{MeV}$ and $m_{u}, m_{d} \sim 3-6 \mathrm{MeV}$ than the Iowa State model's values of 405 and $346 \mathrm{MeV}$ respectively.

\section{THE BREIT EQUATION MODEL OF BRAYSHAW}

\section{A. Definition of The Model and Comparison of Structure with Two-Body Dirac} Approach

Brayshaw [50]treats quarkonium with the aid of the Breit equation and an interaction Hamiltonian with five distinct parts, four of which are independent. As usually done for the Breit equation the times associated with each particle are identified or related in some favored frame (normally c.m.) selected so that the relative time does not enter the potential. In that frame Brayshaw uses the equation

$$
H \Psi=\left(H_{0}+H_{C}+H_{B}+H_{S}+H_{I}+H_{L}\right) \Psi=w \Psi
$$

in which $H_{0}$ is the free Breit Hamiltonian

$$
H_{0}=\boldsymbol{\alpha}_{1} \cdot \mathbf{p}-\boldsymbol{\alpha}_{2} \cdot \mathbf{p}+\beta_{1} m_{1}+\beta_{2} m_{2}
$$


while $H_{C}$ and $H_{B}$ are a Coulomb and an associated Breit interaction

$$
\begin{aligned}
& H_{C}=\frac{c_{1}}{r} \\
& H_{B}=-\frac{c_{1}\left(\boldsymbol{\alpha}_{1} \cdot \boldsymbol{\alpha}_{2}+\boldsymbol{\alpha}_{1} \cdot \hat{\mathbf{r}} \alpha_{2} \cdot \hat{\mathbf{r}}\right)}{2 r} .
\end{aligned}
$$

As indicated in our discussion about the Salpeter equation in Section(VI), this part of the interaction comes from the vector portion of the kernel. The author acknowledges the difficulties associated with the Breit interaction, pointing out that the radial equation has a singularity at a radial separation of $r_{0}=-c_{1} / w>0$. He bypasses Breit's proposal that this interaction be used only in first order perturbation theory by using only positive energy spinors in his variational procedures. We point out that this was not necessary in our approach since the hyperbolic structure of our eight basic interactions avoids problems inherent in Breit's formulation [9]. In particular, it avoids appearance of midpoint singularities. Unfortunately, just like the Wisconsin group, having avoided the pitfalls of the Breit equation, he uses his replacement without testing whether or not his formalism would yield the standard QED results numerically if he limited his interaction to the usual Coulomb interaction. Once again such a test would (if successful) help eliminate the possibility that the wave equation introduces spurious physics.

In Eq.(8.1), $H_{L}$ is a long range confining portion which incorporates the requirement that the wave function vanish identically for radial separations $r>a$ with a boundary condition at $r=a$. Brayshaw argues for this term over and above a linear confinement piece on the grounds that at some separation $r_{p}$ corresponding to a threshold energy $E_{p}$, production of $q \bar{q}$ pairs should become energetically favorable. His radial parameter $a$ plays the role of $r_{p}$ in specifying the range at which such effects (among others) dominate confinement. He expects that $a$ is on the order of $\langle r\rangle$ for the light quark mesons while wave functions for the heavy quark mesons would have fallen to zero for $r<<a$. When introducing the explicit form of his linear confinement potential, the author finds that it cannot simply be added as a Lorentz scalar to the Hamiltonian since such a term produces far too large a mass shift for the light quark systems. Instead he chooses

$$
H_{I}=c_{2}\left(\beta_{1}+\beta_{2}\right) r
$$

which he shows contributes very weakly for the light quark systems, while contributing significantly for the heavy quark systems with an intermediate contribution for the hydrogen- 
like intermediate mass mesons. Unfortunately, however, we note the important fact that the Lorentz transformation character of this confining interaction is ambiguous, being neither scalar $\left(\sim \beta_{1} \beta_{2}\right)$ nor (time-like) vector $\left(\sim 1_{1} 1_{2}\right)$.

Finally Brayshaw introduces a special short range attractive piece solely in order to obtain a good fit to the pion and kaon. Instead of a spin-dependent contact term used in a number of semirelativistic approaches $[51,70,71]$ he uses

$$
H_{S}=H_{B}\left(1_{1} 1_{2}+\beta_{1} \beta_{2}\right) \frac{c_{4} r \theta(b-r)}{2\left(m_{1}+m_{2}+c_{4}\right)}
$$

This term resembles a cross term between a linear confinement piece and the Breit term that might emerge from some sort of iteration. The short range character of this partscalar, part-vector interaction is specified through taking $b<<a$. In contrast, our approach possesses a short range spin-spin interaction that is quantum mechanically well defined and which arises straightforwardly from the Schrödinger reduction of our Dirac equations. We do not need to add it in by hand.

\section{B. Comparison of Fit with that of Constraint Approach}

In spite of its ad hoc nature, we have included the procedure of Brayshaw among our comparisons because it turns out that his resultant fit for the 56 mesons (that overlap with our fit) is quite good, just slightly worse than our fit. In Table IV we include in the fourth column the fit we would obtain with our model if we included only the 56 mesons that our fit has in common with Brayshaw's. On a meson by meson basis we compare by using incremental $\chi^{2}$ values.

Of the 56 mesons in the table, our fits are closer to data in only 26, although overall our fit is better. However, this overall difference may not be as significant as in the previous examples because here we did not use identical fitting procedures for both models. Brayshaw's $R$ values for the two upsilon, the one charmonium, the $K^{*}, \phi$ and $\rho-\pi$ triplet $P$ multiplets are $0.47,0.34,0.32,0.55,0.25,0.19$ and are distinctly different from our values of $0.66,0.69,0.39$,$0.71,-0.25,-5.67$ and the experimental numbers of $0.66,0.61,0.48,0.09,-0.97,-0.4$. Although the constraint/Adler-Piran combination is distinctly better than the Breit/Brayshaw approach for the heavier mesons, both give poor $R$ results for the lighter mesons. All of his light spin-

orbit multiplets have masses that increase monotonically with $j$, unlike the pattern of the 
experimental numbers. Although our results show a non-monotonic pattern that pattern also differs from that of the data. Note that the details of our patterns are greatly influenced by the presence of the scalar potential. Brayshaw's approach includes (see $H_{S}$ ) a partial Hamiltonian that governs intermediate range behavior, in which time-like and scalar interactions contribute equally. This may be responsible for the difference between his montonic pattern and that displayed by the data.

Comparing his ${ }^{3} P$ averages $\left[5\left({ }^{3} P_{2}\right)+3\left({ }^{3} P_{1}\right)+1\left({ }^{3} P_{0}\right)\right] / 9$ to the ${ }^{1} P_{1}$ mesons for the charmonium, $K^{*}$, and $\rho-\pi$ systems we find the following three pairs of numbers: 3.517,3.498;1.335,1.355;1.251,1.202. Comparison to our numbers of $3.519,3.520 ; 1.435,1.421 ; 1.434,1.411$ and the experimental numbers of 3.526,3.525;1.402,1.375;1.231,1.303 shows that our approach gives better agreement for the heavier mesons, his somewhat better for the lighter while both do about the same for the $K^{*}$.

His values of the hyperfine splittings are $118,100,143,158,410,636 \mathrm{MeV}$ for the two charmonium multiplets, and the $D-D^{*}, D_{s}-D_{s}^{*}, K-K^{*}, \pi-\rho$ pairs. Comparing with the experimental splittings of $117,92,142,144,398,627 \mathrm{MeV}$ shows a clear pattern of excellent to good results for the heaviest, lightest, and the intermediate more hydrogen-like mesons. Our results are $151,79,133,145,416,647 \mathrm{MeV}$. Our ground state charmonium result is not nearly as good as Brayshaw's while for the others we have about the same quality of fit. It may be that his choice of $H_{S}$ rectifies the problem our treatment encounters. But, the disadvantage of this is that his $R$ values for the heavy mesons are worse. This effect appears to be similar to the trouble we encountered, mentioned in our discussion of Table I in Sec. IVA.

For the radial excitations, the four upsilon states in the data portion of the table occur at intervals of $563,332,225 \mathrm{MeV}$ while the three charmonium triplet states and the two charmonium singlet states occur at intervals of 589,354,614 MeV. The pion excitation is 1160 $\mathrm{MeV}$. The corresponding Brayshaw intervals are 555,335,320,551,566,569,888 MeV while our intervals are $572,337,257,564,395,636,1403 \mathrm{MeV}$. With the exception of the second radial triplet upsilonium and charmonium excitation intervals, the fits of both models are of about the same quality. Note that excited pion predictions bracket the experimental results. This appears to be a common feature of the radial and orbital excitations of the light quark mesons, with his results on average closer to the experimental values. Our results are, on average, better for the heavier mesons. 
However, his apparently good fit emerges from a potential structure that has ambiguous Lorentz transformation properties. The potentials are chosen in a patchwork manner using the 5 parameters $a, c_{1}, c_{2}, c_{3}, c_{4}$ (he sets $\left.b=a / 10\right)$. In terms of Lorentz transformation properties his scheme uses four invariant functions (scalar, time-like, electromagnetic like and mixed $\left(H_{S}, H_{B}\right.$ and $\left.\left.H_{I}\right)\right)$. The Adler-Piran potential that we use has only two invariant functions corresponding to scalar and electromagnetic like interactions. The constraint approach is not a patchwork; instead its wave equation itself (once $\mathcal{A}$ and $S$ are chosen) fixes the spin, orbital and radial aspects of its potential and its spectra. We also note that just as in the case of the Wisconsin model, Brayshaw has not tested the nonperturbative

reliability of his equation. On the other hand an important result of his approach is that the $u, d$ quark masses required for his fit are very small $(10 \mathrm{MeV})$ and significantly closer to the current quark mass values than ours. His strange quark mass (200 MeV) is also closer to the proposed current quark mass values than our value.

The most important warning provided by Brayshaw's approach is that an ad hoc structure with ambiguous Lorentz properties can do so well at fitting the spectrum.

\section{THE SEMIRELATIVISTIC MODEL OF GODFREY AND ISGUR}

\section{A. Definition of The Model and Comparison of Structure with Two-Body Dirac}

\section{Approach}

We begin with a general discussion of Semirelativistic Quark Models (with and without full Relativistic Kinematics). We term a "semirelativistic quark model" one that uses a two-body wave equation that takes one of the following three forms in the c.m. frame:

$$
\begin{aligned}
\left(\mathbf{p}^{2}+\Phi\left(\mathbf{r}, \mathbf{s}_{1}, \mathbf{s}_{2}\right)\right) \psi & =\left(w-m_{1}-m_{2}\right) \psi \\
\left(\sqrt{\mathbf{p}^{2}+m_{1}^{2}}+\sqrt{\mathbf{p}^{2}+m_{2}}+\Phi\left(\mathbf{r}, \mathbf{s}_{1}, \mathbf{s}_{2}\right)\right) \psi & =w \psi \\
\left(\mathbf{p}^{2}+\Phi\left(\mathbf{r}, \mathbf{s}_{1}, \mathbf{s}_{2}\right)\right) \psi & =b^{2}(w) \psi
\end{aligned}
$$

In each of these equations $\mathbf{p}^{2}$ is the square of the c.m. relative momentum while $\Phi\left(\mathbf{r}, \mathbf{s}_{1}, \mathbf{s}_{2}\right)$ is an effective potential which includes central, spin-orbit, spin-spin, tensor and possibly Darwin terms. In each, the wave function has four components with no coupling to lowerlower components. The most important difference between the first form and the others is 
that the latter two have exact relativistic kinematics. The former is almost always called a nonrelativistic quark model although strictly speaking almost all spin dependences (at least those that arise from vector and scalar interactions) vanish in the nonrelativistic limit. These equations differ from the Two-Body Dirac equations and the Breit and instantaneous BetheSalpeter approaches primarily in that their spin-dependences are put in by hand, abstracted from the Fermi-Breit reductions of the Breit and instantaneous Bethe-Salpeter approaches. For Coulomb-like potentials originating in the Coulomb Gauge, these terms contain singular potentials. Consequently they must either be treated purely perturbatively (thus ruling out application to the light quark mesons) or through the introduction of smoothing parameters that may or may not be features of the actual potential. The two-body Dirac equations of constraint dynamics, like their one-body cousin, have a natural smoothing mechanism potential dependent denominators in the spin-dependent and Darwin terms of the resultant Schrodinger-like form - that eliminates the necessity for ad hoc introduction of such terms. The Breit equation may also possess a natural smoothing mechanism, but a nonperturbative treatment of it leads to erroneous results in QED [5]. The instantaneous Salpeter equation may have a natural smoothing mechanism, but has not been tested nonperturbatively for QED even though the equation is over 50 years old. Authors who have attempted to use these types of semi-relativistic equations to treat the entire meson spectrum include Lichtenberg [70](the third type), Stanley and Robson [71] and Godfrey and Isgur [51] (the second type), and Morpurgo, Ono, and Schöberl [46](the first type). Each of these authors ignore the spin-independent part of the Fermi-Breit interaction. This neglect is not justifiable since this part of the interaction will have an effect on $S$ states that is significantly different from its effect on non $S$-states, being normally short ranged compared with the rest of the central force part of the problem. In this paper, we select one of these models for our final comparison, the model of Godfrey and Isgur, since this model, even though already 18 years old, is by far the most often cited in recent experimental works and theoretical papers on rival approaches.

As we have said, Godfrey and Isgur assume a semi-relativistic wave equation of the second type possessing exact relativistic kinematics but through the inconvenient sum-of-squareroots form. They then determine the form of interaction in the following way. They assume that the confining piece of the interaction is a world scalar. They modify the Coulomb potential with the aid of a smoothing function. At the same time they appear to ignore 
the Darwin term (e.g. the spin independent contact term present in the one-body limit) in the on-shell reduction of the $q \bar{q}$ scattering amplitude. Although they modify the short range part of their interaction with the aid of a smearing function, this modification does not compensate for the ignored Darwin term. We have shown elsewhere [28], [30] that the Darwin interactions for scalar and vector interactions lead, through a canonical transformation to the quadratic local terms $S^{2}$ and $\mathcal{A}^{2}$ that appear in our equations. Since the authors have ignored this part of the Darwin interactions their results contain none of the dynamical consequences of the $S^{2}$ or $-\mathcal{A}^{2}$ pieces. What portion of the Darwin term they include they parametrize separately just as they do the other portions of the Fermi-Breit interaction. These terms include the spin-spin contact term, the spin-orbit terms, and the tensor terms. In our opinion, this patchwork way of handling the physics blurs the relativistic significance of their quark model. In our two-body Dirac equations the Darwin portion and each of the spin-dependent portions is tied directly to and fixed by the Lorentz forms $L\left(x_{\perp}\right), \mathcal{G}\left(x_{\perp}\right)$ of the interaction which are in turn set by the $S, \mathcal{A}$ invariant potentials. In QED these fixed terms yield the correct spectrum with no additional parameters needed to adjust their relative sizes.

In addition to bypassing the problems of singular spin-dependent terms by assuming a smoothing parameter, Godfrey and Isgur include nonlocal (momentum-dependent) potentials by replacing the mass dependent $m_{i}^{-1}$ in the Fermi-Breit term by $\left(\mathbf{p}^{2}+m_{i}^{2}\right)^{-1 / 2}$. They claim that this is necessary because the Fermi-Breit reduction (or the on-shell $q \bar{q}$ scattering amplitude in c.m.) does not adequately express the full momentum dependence (or nonlocal nature) of the potential. While this might be true, we have found that such nonlocal behavior is not necessary to obtain very good results either in lowest order QED or in the quark model.

Like the Adler-Piran potential that we use in our approach, their potential includes a running coupling constant. In fact, by convolving a parametric Gaussian fit to the running coupling constant with the $\frac{1}{\mathbf{q}^{2}}$, they obtain their desired smoothing of the Coulomb potential, thus killing two birds with one stone. In addition, they are able to treat the zero isospin mesons like the $\eta$ and $\eta^{\prime}$ by including a phenomenological annihilation term. We leave out this term in our results of Table I-IV and in our comparison with the results of Godfrey and Isgur in Table V. Lichtenberg [70] has compared an earlier version of our quark model for the meson spectrum with that of Godfrey and Isgur. The potential we used in that earlier 
version was the one-parameter Richardson potential, with the confinement piece chosen to be one-half time-like vector and one-half scalar. As Lichtenberg pointed out, Godfrey and Isgur obtained significantly better agreement with the data than we did. He states that this is because they use significantly more parameters than we do including four in the potential and six to describe relativistic effects, ten altogether, compared to our one. However, we do not believe that as a general rule the number of parameters that appear in the potential is, in itself, of as much significance as how these parameters are distributed. For example, in our present and previous models there are two invariant functions, $\mathcal{A}$ and $S$ related to the single nonrelativistic (Adler-Piran) $V_{A P}$ that itself depends on two parameters. These parametric functions are not entirely independent, being related by Eqs.(3.1,3.8a,3.8b). Specifying their form fixes both spin-independent and spin-dependent parts of the quasipotential $\Phi_{w}$. We might say that our formalism has 5 quark mass parameters and two parametric functions. Increasing the number of parameters that $\mathcal{A}, S$ depend on may or may not increase the goodness of the fit. According to our way of counting, Godfrey and Isgur have independent parametric functions for the two spin-orbit parts of the potential, the spin-spin contact part, the tensor part, the scalar potential, and the spin-independent part of the vector potential, altogether 6 parametric functions. From our way of counting the number of parameters the number of parametric functions would not increase no matter how many parameters are included in fixing the functional form of each of these six functions. Likewise, in our case, no matter how many parameters we use in fixing $\mathcal{A}, S$ there are only two independent parametric functions. Our approach is distinct from that of Godfrey and Isgur in that we do not alter the functional form at the level of the spin-dependence but rather at the level of the kernels.

Finally, before we compare our present work with that of Godfrey and Isgur, we note that our present model differs from our earlier one used by Lichtenberg in his comparison of the two approaches. Our present treatment differs in its replacement of the Richardson potential by the Adler-Piran potential. The intermediate range form of the A-P potential is closely tied to an effective field theory related to QCD and is therefore superior to Richardson's ansatz. Furthermore, in calculations based on our earlier treatment we ignored the tensor coupling and unequal mass spin-orbit difference couplings which we explicitly include in the present calculations. We have also corrected a defect in the decoupling we used between the upper-upper and lower-lower components of the wave functions for spin-triplet states in our 
older treatment.

\section{B. Comparison of Fit with that of Constraint Approach}

We now compare the fit given by our model to that provided by the model of Godfrey and Isgur.

In Table $\mathrm{V}$ we display in the fourth column the fit we would obtain with our model if we included only the 77 mesons that our fit has in common with that of Godfrey and Isgur. We then compare the fits by examining the incremental $\chi^{2}$ values for each meson. (In an RMS comparison they would obtain about 63 compared to our value of 79).

For the 77 mesons in their table, our fits are closer to data in only 32; overall their fit is better. Generally speaking our results are better on the newer mesons while their fit is better on the older mesons. A detailed comparison reveals the following. Their $R$ values for the two upsilon, the one charmonium, the $K^{*}, \phi$ and $\rho-\pi$ triplet $P$ multiplets are 0.29,0.50,0.57,0.36,0.42,0.47 and are distinctly different from our values of 0.68,0.76,0.41,$0.66,-0.21,-4.00$ and the experimental numbers of $0.66,0.61,0.48,0.09,-0.97,-0.4$. As was true for the Brayshaw analysis, the constraint/Adler-Piran combination gives a distinctly better fit than the Isgur-Wise approach for the heavier mesons, while both give poor results for the lighter mesons. As was the case for Brayshaw's spectrum, none of their light multiplets are inverted, whereas although ours are inverted they are not inverted in the same way as the experimental numbers are. Again, our inversions are due to the action of the scalar potential. Godfrey and Isgur include a time-like contribution in the spin-orbit part of their Hamiltonian. This may be responsible for their lack of the partial inversion that appears in the data.

Computing their ${ }^{3} P$ averages $\left[5\left({ }^{3} P_{2}\right)+3\left({ }^{3} P_{1}\right)+1\left({ }^{3} P_{0}\right)\right] / 9$ along with the ${ }^{1} P_{1}$ mesons for the charmonium, $K^{*}$ and $\rho-\pi$ system we find the following three pairs of numbers: 3.524,3.520;1.392,1.340;1.262,1.220. Comparison with our numbers of $3.519,3.520 ; 1.424,1.411 ; 1.419,1.397$ and the experimental numbers of 3.526,3.525;1.402,1.375;1.231,1.303 shows the constraint approach giving slightly better numbers for the heavier mesons and the $K^{*}$ while the Godfrey-Isgur results are somewhat better for the lighter mesons. Their ${ }^{3} D$ average $\left[7\left({ }^{3} D_{2}\right)+5\left({ }^{3} D_{1}\right)+3\left({ }^{3} D_{1}\right)\right] / 15$ and their ${ }^{1} D_{2}$

meson for the $K^{*}$ are $1.795,1.780 \mathrm{MeV}$ while our results and the experimental results are 
1.873,1.879 and 1.774,1.773 MeV respectively. Our results are relatively closer to one another while theirs are closer to the data in an absolute sense. This is indicative of the general trend of our orbitally excited light mesons being somewhat high. We suspect that this is due to the $S^{2}$ behavior becoming dominant at longer distance, changing the behavior of the confining potential in the effective Schrödinger-like equation from linear to quadratic.

Their values of the hyperfine splittings are $130,60,160,150,430,130,620,150,120 \mathrm{MeV}$ for the two charmonium multiplets, and the $D-D^{*}, D_{s}-D_{s}^{*}$, two $K-K^{*}$, and three $\pi-\rho$ pairs. Comparison with the experimental splittings of $117,92,142,144,398,-48,627,165,354$ $\mathrm{MeV}$ and our results of $150,78,133,145,403,208,645,239,166 \mathrm{MeV}$ demonstrates that while our results are closer than theirs for most of the newer mesons and the $K-K^{*}$, their results are more in line for most of the older mesons. Again this shows a pattern of our method overestimating the radially excited states of the light mesons.

Let us see if this trend of overestimation by the constraint approach continues for the radial excitations of fixed quantum numbers. The six upsilon states in the data portion of the table occur at intervals of $563,332,225,285,154 \mathrm{MeV}$ while the three charmonium triplet states and the two charmonium singlet states occur at intervals of $589,354,614 \mathrm{MeV}$ whereas the three singlet $K$ and the two triplet $K^{*}$ states occur at intervals of 977,370 and $520 \mathrm{MeV}$. Finally the three pion and three rho excitations occur at 1160,495 and $698,654 \mathrm{MeV}$. The corresponding Isgur-Wise intervals are $540,350,280,250,220,580,420,650,980,570,680,1150,580,680,550 \mathrm{MeV}$ compared to our intervals of $570,336,256,213,186,561,393,633,1099,495,894,1383,634,986,561 \mathrm{MeV}$. Again we encounter a pattern of our results being more accurate overall for the newer mesons while theirs are more accurate for the older ones (with our results too large for all of the older ones).

Primarily what we learn from this comparison is that not only does the scalar interaction lead to partial triplet inversions for the lighter mesons but also yields radial and orbital excitations that are too high for a related reason: the presence of the $S^{2}$ term in the effective potential. On the other hand, as Godfrey and Isgur themselves point out, their treatment of the relativistic effects is schematic, with no wave equation involved, allowing an uncontrolled approach in which there are no tightly fixed connections among the various spin-dependent and spin-independent parts of the effective potential $\Phi$.

An important feature of our approach that differs significantly from the model of Godfrey 
and Isgur (as well as those of the Wisconsin and Iowa State groups) is the size of its resulting light quark masses. Our $u, d$ quark masses are about a factor of four or five smaller than theirs, significantly closer to the current algebra values. Godfrey and Isgur argue that since a constituent quark model requires dressed quarks of a finite size (to avoid singular potentials in their wave equation among other reasons) one should not expect the model quarks to have current-quark masses. We argue that a properly structured relativistic wave equation should not require finite quark sizes. Similar remarks have been made historically to justify tampering with the wave equation in QED to avoid treating singular terms. However, in QED those terms are perturbative artifacts. In fact, in the constraint equations for QED, they arise from premature weak-potential approximation to terms that are actually wellbehaved at the origin. Similarly, when we apply the constraint approach to QCD we need no size parameters.

Finally we mention what we consider the major theoretical shortcoming in the approach of Godfrey and Isgur. The formalism that they use gives very good results on the hyperfine splittings for light and heavy mesons. However, it is unknown if this is an artifact of their smearing factors and the introduction of relativistic momentum dependent corrections to the potentials (that is, through the replacement of quark masses $m$ by $\sqrt{p^{2}+m^{2}}$ ) needed to modify the singular nature of the potentials that they start with.

It would be of interest to test the wave equation used by Godfrey and Isgur numerically with $\mathcal{A}=-\alpha / r$ and $S=0$ for positronium to see if any of their successes with mesonic hyperfine splittings are reflections of corresponding nonperturbative successes in QED. If their method were not able to obtain an acceptable fit to the QED spectral results through order $\alpha^{4}$, then the legitimacy of its fits in QCD would be seriously called into question. Without such tests one could not be sure whether the method they employ to avoid the singular potentials has distorted the dynamics. The constraint approach has passed this test in that without introducing additional parameters it does faithfully reproduce the correct spectral results in QED. 


\section{CONCLUSION AND WARNINGS ABOUT THE DANGERS OF "RELATIVIS- TIC" AND “NONRELATIVISTIC" SPECTRAL FITS}

In this paper, we have investigated how well the relativistic constraint approach performs in comparison with selected alternatives when used to produce a single fit of experimental results over the whole meson spectrum. This approach is distinguished from others by its foundation - a set of coupled, compatible, fully covariant wave equations whose nonperturbative numerical solution yields the mass spectrum along with wave functions for the $q \bar{q}$ meson bound states. Its virtue - generation of fully covariant spin structures - also serves to restrict and relate plausible interaction terms just as the ordinary single-particle Dirac equation determines relations among Pauli spin dependences and fixes the proper strength of the Thomas precession term in electrodynamics. The dynamical structures of the constraint approach were originally discovered in classical relativistic mechanics but have since been verified for electrodynamics through diagrammatic summation in quantum field theory in the field-theoretic eikonal approximation [27].

To use such relativistic equations to treat the phenomenological chromodynamic $q \bar{q}$ bound-state, one must construct a relativistic interaction that possesses the limiting behaviors of QCD. In our approach we have done this by using the nonrelativistic static Adler-Piran potential to construct a plausible relativistic interaction that regenerates the AP potential as its nonrelativistic limit. In our equations, this process generates a host of accompanying interaction terms. When describing these interactions, one must guard against a semantic difficulty in the verbal classification of the various parts of the interaction as "scalar", "vector", "pseudovector" etc. The various formalisms classify these in different ways but in our equations, the meaning of these terms can be readily determined through examining their roles in the defining equation Eq. $(2.26,5.1,5.2,5.4)$. Once these terms have been introduced, the constraint formalism automatically produces a system of important accompanying terms like quadratic terms that dominate at long distance (reinforcing or undermining confinement) or spin dependences that accompany chosen interactions producing level splits that agree or disagree with the experimental results in various parts of the spectrum.

After identification of the relativistic transformation properties of interaction terms the constraint method leaves almost no leeway for fiddling with (unnecessary) cutoffs, etc. Some 
years ago, when applied to the $e^{-} e^{+}$system, its structure proved restrictive enough to rule out within it the presence of postulated anomalous resonances[25, 72]. In recent work on the relation of our equations to the Breit and earlier Eddington-Gaunt equations for electromagnetic bound-states, the method has explicitly demonstrated the importance of keeping spin couplings among pieces of the full 16-component wave-functions whose counterparts are often truncated or discarded in alternative treatments [9, 37].

The fits that we have examined as alternatives fall into different classes: motivated relativistic fits ( constraint vs truncations of standard field-theoretic), ad-hoc relativistic fits, and cautious semirelativistic fits.

Among the relativistic ones, there is a danger exemplified by the Brayshaw model which achieves relative success despite the dubious relativistic nature of its interaction. As always, what makes fits hard to judge at this stage is the ease with which one can achieve apparent success over limited regions of the spectrum using highly-parametrized interactions. We have attempted to avoid this problem by limiting comparisons to published treatments that include both the light and heavy meson portions of the spectrum, not just one of the two sectors. Our choices for comparison are meant to be representative (we do not attempt an exhaustive review) (see [73] for other important treatments).

With the exception of the Iowa State model [49] all of the comparison models fail to test whether or not a nonperturbative treatment of their wave equations would yield the known results if the QCD kernels used were to be replaced by ones appropriate for QED. With the exception of the quark masses obtained by Brayshaw, [50] our light quark masses are substantially closer to the current algebra values than are those produced by the other comparison models. In our application of the constraint approach, it is possible to describe the nonperturbative physics that accommodates a typical size for an effective or constituent quark mass used in the other approaches and which at the same time has the size necessary to account for baryon magnetic moments. Even though our $u$ and $d$ quark masses are small compared with constituent quark masses found in the competing approach, if we compute the expectation value $\left\langle M_{i}(\mathcal{A}, S)\right\rangle$ we find a range that includes those values. We find the range of values for this effective mass from $64 \mathrm{MeV}$ for the pion to $390 \mathrm{MeV}$ for the rho. Its value depends not only on the quantum numbers of the meson but also the flavor of the other quark. For example, for the $D$ meson we find $\left\langle M_{u}(\mathcal{A}, S)\right\rangle=190 \mathrm{MeV}$ whereas for the $B$ we obtain $258 \mathrm{MeV}$. 
Finally, some authors have even produced unabashedly nonrelativistic fits. They claim to obtain good fits to the meson spectrum through the use of variants of the nonrelativistic quark model (NRQM). [46], [74]. These authors even claim success at fitting the light quark mesons for which the assumptions $T<<m c^{2},|V|<<m c^{2}$ of the nonrelativistic Schrödinger equation are patently false. What can account for the apparent success of the NRQM?

Morpurgo states [46] that the various potential models, including the nonrelativistic quark model, are merely different parametrizations of an underlying exact QCD Lagrangian description. That is, all use essentially the same spin and flavor structures. For example, for the mesons one can derive a "parametrized mass" with general form (for the present discussion restricted to $\left.\pi, K, \rho, K^{*}\right)$

$$
\text { "parametrized mass" }=A+B\left(P_{1}^{s}+P_{2}^{s}\right)+C \boldsymbol{\sigma}_{1} \cdot \boldsymbol{\sigma}_{2}+D\left(P_{1}^{s}+P_{2}^{s}\right) \boldsymbol{\sigma}_{1} \cdot \boldsymbol{\sigma}_{2}
$$

in which $P_{i}^{s}$ is the projector onto the strange quark sector. These authors say that this structure although typical of an NRQM description, follows from QCD itself. They state that the form Eq.(10.1) is common to all of the relativistic or semirelativistic quark models. They assert that any one of them can be successful but not superior to any other, if it merely reproduces the spin flavor structure of the general parametrization. Thus, from their point of view selection of the "best" model is entirely a matter of taste and simplicity. We disagree with this assessment for the following reasons. First, the kinetic and potential parameters have significances beyond simply producing a fit for the two-body bound-state sector in isolation. When the spin-flavor structure in (10.1) appears in the constraint approach, its accompanying constituent quark masses turn out to be closer to the current-quark masses than those produced by most other approaches while the constraint method requires only two parametric functions to be used beyond the parameters of the constituent quark masses. The constraint scheme successfully uses one set of these parametric functions for the entire spectrum of meson states including the radial as well as orbital excitations. But most importantly, within the bound-state spectrum itself, in our relativistic approach even though superficially sharing the basic spin-flavor structure (10.1), all potentials do not fare equally well. The essential point is that even in the simplest form of our equations, the parametrization is different from that given in the Morpurgo form in that its parameters A,B,C,D, are 
themselves dependent on the energy operator on the left hand side. When that happens, some relativistic potentials do better than others. In particular, of those we investigated, the potential that works the best (the Adler-Piran potential) is one possessing many of the features important in lattice QCD calculations (e.g. linear and subdominant logarithmic confining pieces). The combination of the constraint approach with the Adler-Piran potential embodies more of the important physical effects contained in QCD-related effective or numerical field theories.

Can one understand the apparent successes of the NRQM fits by starting from the relativistic treatments? Some authors [75] and [76] have used bounds on the kinetic square-root operator $\sqrt{\mathbf{p}^{2}+m^{2}}$ to attempt to understand the apparent success of the nonrelativistic potential models for relativistic quark-antiquark states. Instead, we will give an explanation that starts directly from the relativistic constraint approach.

Some years ago, Caswell and Lepage [77] rewrote a relativistic constraint equation in an effective nonrelativistic Schrodinger-like form. Here, we do the opposite and recast the NRQM Schrodinger equation in a form resembling the constraint equation. As we have seen our two-body Dirac equations lead to an effective Schrödinger-like equation of the form

$$
\left[p^{2}+\Phi_{w}\left(x_{\perp}, \sigma_{1}, \sigma_{2}\right)\right] \psi=b^{2}(w) \psi
$$

In the c.m. system this becomes

$$
\left[\mathbf{p}^{2}+\Phi_{w}\left(\mathbf{r}, \boldsymbol{\sigma}_{1}, \boldsymbol{\sigma}_{2}\right)\right] \psi=b^{2}(w) \psi
$$

Even though the stationary state nonrelativistic Schrödinger equation

$$
\left[\frac{\mathbf{p}^{2}}{2 \mu}+V\left(\mathbf{r}, \boldsymbol{\sigma}_{1}, \boldsymbol{\sigma}_{2}\right)\right] \psi=E_{B} \psi
$$

has a similar form, the corresponding structures in each have entirely different physical significances. For example, in Eq.(10.4), the vectors $\mathbf{p}$ and $\mathbf{r}$ are nonrelativistic quantities in contrast with their counterparts in the constraint approach that appear in the relativistic equation in the c.m. system. One can easily manipulate the nonrelativistic Schrödinger equation into a form similar in appearance to the constraint Schrödinger form by multiplying both sides of the equation by $2 \mu$ and adding $b^{2}(w)-2 \mu E_{B}$ to both sides. The result is

$$
\left[\mathbf{p}^{2}+\Phi_{w}\left(\mathbf{r}, \boldsymbol{\sigma}_{1}, \boldsymbol{\sigma}_{2}\right)\right] \psi=b^{2}(w) \psi
$$


in which

$$
\Phi_{w}\left(\mathbf{r}, \boldsymbol{\sigma}_{1}, \boldsymbol{\sigma}_{2}\right)=2 \mu V\left(\mathbf{r}, \boldsymbol{\sigma}_{1}, \boldsymbol{\sigma}_{2}\right)+b^{2}(w)-2 \mu E_{B}
$$

In numerical calculations the $\mathbf{p}$ operator and $\mathbf{r}$ variable are treated in the same manner in calculations based on both the relativistic constraint equation and the nonrelativistic equation. But as we have seen, they have different physical significances in each equation. When used to fit parts of the meson spectrum, the apparent success of the NRQM from this point of view is then due to its incorporation of variables numerically indistinguishable from their covariant versions together with a potential that fortuitously coincides (for a limited range of states) with a covariant one modified by an energy dependent constant term that varies from state to state.

\section{APPENDIX A: PAULI-FORM OF THE TWO-BODY DIRAC EQUATIONS FOR $\phi_{+}=\psi_{1}+\psi_{4}$ AND THEIR RADIAL FORMS}

Reference [53] sets out Two-Body Dirac Equations containing general covariant interactions along with their accompanying Schrödinger-like forms. The general interactions consist of the eight Lorentz invariant forms corresponding to scalar, time and space-like vector studied here along with five others: pseudoscalar, time- and space-like pseudovector, axial and polar tensor. When Eq.(2.26) is written in terms of the four four-component spinors $\psi_{1 \ldots 4}$ it decomposes into eight coupled equations. In [53] Long and Crater showed how these may be rearranged in Pauli-form or Schrodinger-like equations in terms of the combination $\phi_{+}=\psi_{1}+\psi_{4}$ in the process providing a simpler coupling scheme than that used in [25] which involves coupled equations between $\psi_{1}$ and $\psi_{4}$. Eq.(4.24) of reference [53] yields the following equation (simplified here for electromagnetic-like interactions $\left(\partial J \equiv \frac{\partial E_{1}}{E_{2}}=-\partial G\right)$ and scalar interactions alone):

$$
\begin{gathered}
{\left[E_{1} D_{1}^{-+} \frac{1}{E_{1} M_{2}+E_{2} M_{1}}\left(M_{2} D_{1}^{++}-M_{1} D_{2}^{++}\right)\right.} \\
\left.+M_{1} D_{1}^{--} \frac{1}{E_{1} M_{2}+E_{2} M_{1}}\left(E_{2} D_{1}^{++}+E_{1} D_{2}^{++}\right)\right] \phi_{+} \\
=\left(E_{1}^{2}-M_{1}^{2}\right) \phi_{+}
\end{gathered}
$$

in which the kinetic-recoil terms appear through the combinations:

$$
D_{1}^{++}=\exp \mathcal{G}\left[\sigma_{1} \cdot p+\frac{i}{2} \sigma_{2} \cdot \partial\left[L+\mathcal{G}\left(1-\sigma_{1} \cdot \sigma_{2}\right)\right]\right]
$$




$$
\begin{aligned}
D_{2}^{++} & =\exp \mathcal{G}\left[\sigma_{2} \cdot p+\frac{i}{2} \sigma_{1} \cdot \partial\left[L+\mathcal{G}\left(1-\sigma_{1} \cdot \sigma_{2}\right)\right]\right] \\
D_{1}^{-+} & =\exp \mathcal{G}\left[\sigma_{1} \cdot p+\frac{i}{2} \sigma_{2} \cdot \partial\left[-L+\mathcal{G}\left(1-\sigma_{1} \cdot \sigma_{2}\right)\right]\right] \\
D_{1}^{--} & =\exp \mathcal{G}\left[\sigma_{1} \cdot p+\frac{i}{2} \sigma_{2} \cdot \partial\left[L-\mathcal{G}\left(1+\sigma_{1} \cdot \sigma_{2}\right)\right]\right]
\end{aligned}
$$

Manipulations using both sets of Pauli-matrices then lead to the form presented in the text in Eq.(2.35).

We obtain the radial forms of Eq.(2.35) that we use for our numerical solution for the general fermion-antifermion system by forming standard matrix elements of spin-dependent operators (see Appendix C of Ref.([25] )). We start from the general wave function of the form

$$
\psi_{i j m}=\sum_{l, s} c_{i l s} R_{i l s j} \mathcal{Y}_{l s j m} ; i=1,2,3,4
$$

in which $R_{i l s j}=\frac{u_{i l s j}}{r}$ is the associated radial wave function and $\mathcal{Y}_{l s j m}$ is the total angular momentum eigenfunction. In terms of $\mathcal{D}=E_{1} M_{2}+E_{2} M_{1}$ the corresponding radial forms then become

$$
\begin{gathered}
s=0, \quad j=l \\
\left\{-\frac{d^{2}}{d r^{2}}+\frac{j(j+1)}{r^{2}}+2 m_{w} S+S^{2}+2 \epsilon_{2} \mathcal{A}-\mathcal{A}^{2}\right. \\
-(2 \mathcal{G}-\log (\mathcal{D})+\mathcal{G}+L)^{\prime}\left(\frac{d}{d r}-\frac{1}{r}\right) \\
\left.-\frac{1}{2} \nabla^{2}(L+4 \mathcal{G})-\frac{1}{4}(-L-2 \mathcal{G}+2 \log (\mathcal{D}))^{\prime}(-L-4 \mathcal{G})^{\prime}\right\} u_{j 0 j} \\
+\exp (-\mathcal{G}-L) \frac{w\left(m_{1}-m_{2}\right)}{\mathcal{D}}(-\mathcal{G}+L)^{\prime} \frac{\sqrt{j(j+1)}}{r} u_{j 1 j}=b^{2}(w) u_{j 0 j}
\end{gathered}
$$

coupled to

$$
\begin{gathered}
s=1, \quad j=l \\
\left\{-\frac{d^{2}}{d r^{2}}+\frac{j(j+1)}{r^{2}}+2 m_{w} S+S^{2}+2 \epsilon_{2} \mathcal{A}-\mathcal{A}^{2}\right. \\
-(\mathcal{G}-L-\log (\mathcal{D}))^{\prime} \frac{d}{d r}-\frac{L^{\prime}}{r} \\
\left.+\frac{1}{2} \nabla^{2} L+\frac{1}{4}(2 \log (\mathcal{D})+(-L+2 \mathcal{G}))^{\prime} L^{\prime}\right\} u_{j 1 j}
\end{gathered}
$$




$$
+\exp (-\mathcal{G}-J) \frac{\left(\epsilon_{1}-\epsilon_{2}\right)\left(m_{1}+m_{2}\right)}{\mathcal{D}}(-\mathcal{G}+L)^{\prime} \frac{\sqrt{j(j+1)}}{r} u_{j 0 j}=b^{2}(w) u_{j 1 j}
$$

and $s=1, j=l+1$

$$
\begin{gathered}
\left\{\left(-\frac{d^{2}}{d r^{2}}+\frac{j(j-1)}{r^{2}}\right)+2 m_{w} S+S^{2}+2 \epsilon_{2} \mathcal{A}-\mathcal{A}^{2}\right. \\
+\left[\log (\mathcal{D})-2 \mathcal{G}+\frac{1}{2 j+1}(G+L)\right]^{\prime} \frac{d}{d r} \\
{\left[-j \log (\mathcal{D})+\frac{1}{2 j+1}\left(\left(4 j^{2}+j+1\right) \mathcal{G}-\mathcal{G}-L\right)\right]^{\prime} \frac{1}{r}} \\
+\frac{1}{4}\left(-(\mathcal{G}+L)^{\prime 2}\right)+\frac{1}{2 j+1}\left(\left(\frac{1}{2} \nabla^{2} L+\mathcal{G}^{\prime}\left(\frac{2 j-3}{4} \mathcal{G}+\mathcal{G}+L\right)^{\prime}-\frac{1}{2} \log ^{\prime}(\mathcal{D}) L^{\prime}\right)\right\} u_{j-11 j} \\
+\frac{\sqrt{j(j+1)}}{2 j+1}\left\{2[\mathcal{G}+L]^{\prime} \frac{d}{d r}+[(-\mathcal{G}-L)(1-2 j)+3 \mathcal{G}]^{\prime} \frac{1}{r}\right. \\
\left.+\nabla^{2}(L)-L^{\prime}(\log (\mathcal{D})-2 \mathcal{G})^{\prime}\right\} u_{j+11 j}=b^{2}(w) u_{j-11 j},
\end{gathered}
$$

coupled to $s=1, j=l-1$

$$
\begin{gathered}
\left\{\left(-\frac{d^{2}}{d r^{2}}+\frac{(j+1)(j+2)}{r^{2}}\right)+2 m_{w} S+S^{2}+2 \epsilon_{2} \mathcal{A}-\mathcal{A}^{2}\right. \\
+\left[\log (\mathcal{D})-2 \mathcal{G}-\frac{1}{2 j+1}(G+L)\right]^{\prime} \frac{d}{d r} \\
{\left[(j+1) \log (\mathcal{D})-\frac{1}{2 j+1}\left(\left(4 j^{2}+7 j+4\right) \mathcal{G}-\mathcal{G}-L\right)\right]^{\prime} \frac{1}{r}} \\
+\frac{1}{4}\left(-(\mathcal{G}+L)^{\prime 2}\right)-\frac{1}{2 j+1}\left(\left(\frac{1}{2} \nabla^{2} L+\mathcal{G}^{\prime}\left(\frac{2 j+5}{4} \mathcal{G}-\mathcal{G}-L-C\right)^{\prime}+\frac{1}{2} \log ^{\prime}(\mathcal{D}) L^{\prime}\right)\right\} u_{j+11 j} \\
+\frac{\sqrt{j(j+1)}}{2 j+1}\left\{2[\mathcal{G}+L]^{\prime} \frac{d}{d r}+[(-\mathcal{G}-L)(2 j+3)+3 \mathcal{G}]^{\prime} \frac{1}{r}\right. \\
\left.+2 \nabla^{2} L+L^{\prime}(\log (\mathcal{D})-2 \mathcal{G})^{\prime}\right\} u_{j-11 j}=b^{2}(w) u_{j+11 j} .
\end{gathered}
$$

\section{APPENDIX B: NUMERICAL CONSTRUCTION OF MESON WAVE FUNC-}

\section{TIONS}

We obtain from our computer program a numerical wave function $\bar{u}(x)$ normalized so that

$$
\int_{-\infty}^{+\infty} \bar{u}(x)^{2} d x=1
$$


The radial variable is related to $x$ by $r=r_{0} e^{x}$ and the radial wave function $u(r)=$ $\bar{u}(x) e^{-x / 2} / \sqrt{r_{0}}$. Hence

$$
\int_{0}^{+\infty} u(r)^{2} d r=\int_{-\infty}^{+\infty} \bar{u}(x)^{2} d x .
$$

Now let $v_{n}(r)$ be some radial basis functions that are orthonormalized so that

$$
\int_{0}^{+\infty} v_{n}(r) v_{n^{\prime}}(r) d r=\delta_{n n^{\prime}}
$$

Thus

$$
u(r)=\sum_{n=0}^{\infty} u_{n} v_{n}(r)
$$

where

$$
u_{n}=\int_{0}^{+\infty} v_{n}(r) u(r) d r=\int_{-\infty}^{+\infty} \bar{v}_{n}(x) \bar{u}(x) d x .
$$

Note that $\bar{v}_{n}(x)=v_{n}(r) e^{x / 2} \sqrt{r_{0}}$ so that we can compute the $u_{n}$ in a straightforward way. Thus we have as an approximation

$$
\begin{aligned}
u(r) & \doteq \sum_{n=0}^{N} v_{n}(r) \int_{-\infty}^{+\infty} \bar{v}_{n}(x) \bar{u}(x) d x \\
& =\sum_{n=0}^{N} c_{n} v_{n}(r) \equiv w_{N}(r) .
\end{aligned}
$$

Now we use a least squares fit to determine the $c_{n}$. In the limit of large $N$ we have $c_{n} \rightarrow u_{n}$ since we minimize the quantity

$$
\chi^{2} \equiv \int_{-\infty}^{+\infty}\left|\bar{u}(x)-\bar{w}_{N}(x)\right|^{2} d x
$$

For the $v_{n}(r)$ we use harmonic oscillator (Laguerre) functions defined by

$$
v_{n}^{k}(y)=c(n, k) e^{-y^{2} / 2} y^{k} L_{n}^{k-1 / 2}\left(y^{2}\right)
$$

in which $c(n, k)=\sqrt{\frac{2(n !)}{a(n+k-1 / 2) !}}$ is the normalization constant and in terms of $z=y^{2}$

$$
L_{n}^{k-1 / 2}(z)=\frac{e^{z} z^{-k+1 / 2}}{n !} \frac{d^{n}}{d z^{n}}\left(e^{-z} z^{k+n-1 / 2}\right)
$$

So for example 


$$
\begin{aligned}
L_{0}^{k-1 / 2}(z) & =1 \\
L_{1}^{k-1 / 2}(z) & =k+1 / 2-z \\
L_{2}^{k-1 / 2}(z) & =\frac{1}{2}\left[(5 / 2+k-z) L_{1}^{k-1 / 2}(z)-(1 / 2+k) L_{0}^{k-1 / 2}(z)\right. \\
& =\left[(k+3 / 2)(k+1 / 2)-2(k+3 / 2) z+z^{2}\right] / 2 \\
& \ldots \\
L_{n+1}^{k-1 / 2}(z) & =\frac{1}{n+1}\left[(2 n+1 / 2+k-z) L_{n}^{k-1 / 2}(z)-(n+k-1 / 2) L_{n-1}^{k-1 / 2}(z)\right]
\end{aligned}
$$

Thus letting $y=r / a=\alpha e^{x}$ we obtain

$$
\begin{aligned}
\bar{v}_{0}(x) & =c(0, k) \alpha^{k} \exp (x(2 k+1) / 2) \exp \left(-\alpha^{2} e^{2 x} / 2\right) \\
\bar{v}_{1}(x) & =\sqrt{\frac{1}{k+1 / 2} \bar{v}_{0}(x)\left(k+1 / 2-\alpha^{2} e^{2 x}\right)} \\
\bar{v}_{2}(x) & =\sqrt{\frac{2 !}{(k+1 / 2)(k+3 / 2)} \bar{v}_{0}(x)\left[(k+3 / 2)(k+1 / 2)-2(k+3 / 2) \alpha^{2} e^{2 x}+\alpha^{4} e^{4 x}\right] / 2 .} \\
& \ldots \\
\bar{v}_{n}(x) & =\sqrt{\frac{n !}{(k+1 / 2) . .(k+n-1 / 2)}} \bar{v}_{0}(x) \sum_{m=0}^{n}(-)^{m} \frac{(n+k-1 / 2) !}{(n-m) !(k-1 / 2+m) ! m !}\left(\alpha e^{x}\right)^{2 m}
\end{aligned}
$$




\section{APPROACHES TREATED IN THIS PAPER}

$\begin{array}{llllll} & \text { HC-PVA } & \text { Wisconsin } & \text { Iowa State } & \text { Brayshaw } & \text { Godfrey,Isgur } \\ \text { Wave Eqn } & \text { Two-Body Dirac } & \text { Reduced BSE } & \text { Quasipotential Breit } & \text { None } \\ \text { Covariance } & \text { Explicit } & \text { Implicit } & \text { Implicit } & \text { Implicit } & \text { Implicit } \\ \text { Nonperturb. Tests } & \text { Strng. ptnl -QED } & \text { Wk ptnl. } & \text { Str. ptnl. } & \text { Str. ptnl } & \text { Str. ptnl. } \\ \# \text { of Parametric fns } & 2 & 2 & 2 & 3 & 6 \\ \chi^{2} & 101 & 5169 \text { vs } 73 & \text { RMS } 50 \text { vs } 53 & 204 \text { vs } 11185 \text { vs } 105 \\ \text { Locality } & \text { Local } & \text { Non-local } & \text { Non-local } & \text { Local } & \text { Non-local } \\ \text { Running coupling cnst. Yes } & \text { Yes } & \text { Yes } & \text { No } & \text { Yes }\end{array}$

\section{References}

[1] G. Breit, Phys. Rev. 34, 553, 1929

[2] G. Breit, Phys. Rev. 36, 383, 1930

[3] G. Breit, Phys. Rev. 39, 616, 1932

[4] H.A. Bethe and E. E. Salpeter, Quantum Mechanics of One and Two Electron Atoms (Springer, Berlin, 1957).

[5] W. Krolikowski, Acta Physica Polonica, B12, 891 (1981). Nonperturbative treatments of truncated versions of the Breit equation (with just the Coulomb term) have yielded the same results as a perturbative treatment of the same truncations but none of these have included the troublesome transverse photon parts. See J. Malenfant, Phys. Rev. A 43, 1233 (1991), and T.C. Scott, J. Shertzer, and R.A. Moore, ibid 45,4393 (1992)

[6] R. W. Childers, Phys. Rev. D26, 2902 (1982).

[7] C. W. Wong and C. Y. Wong Phys. Lett. B301, 1 (1993).

[8] C. W. Wong and C. Y. Wong Nucl. Phys. A562, 598 (1993).

[9] A generalization of the Breit equation which can be treated nonperturbatively based on constraint dynamics is given in H. W. Crater, C. W. Wong, C. Y. Wong, and P. Van Alstine, Intl. Jour. of Mod. Phys. E 5, 589 (1996)

[10] P.A.M. Dirac, Lectures on Quantum Mechanics (Yeshiva University, Hew York, 1964). 
[11] M. Kalb and P. Van Alstine, Yale Reports, C00-3075-146 (1976),C00-3075-156 (1976); P. Van Alstine, Ph.D. Dissertation Yale University, (1976).

[12] I. T. Todorov, "Dynamics of Relativistic Point Particles as a Problem with Constraints", Dubna Joint Institute for Nuclear Research No. E2-10175, 1976; Ann. Inst. H. Poincare' A28,207 (1978).

[13] L.P. Horwitz and F. Rohrlich, Phys. Rev. D24, 1928 (1981), F. Rohrlich, Phys. Rev. D23 1305,(1981). See also H. Sazdjian, Nucl. Phys. B161, 469 (1979).It is even a nontrivial task to disentangle the two superfluous relative time degrees of freedom from the kinematics for three noninteracting Dirac equations to obtain a collective constraint-like formalism [16],[17].

[14] A. Komar, Phys. Rev. D18, 1881,1887 (1978).

[15] P. Droz-Vincent Rep. Math. Phys.,8,79 (1975).

[16] H. Sazdjian, Annals of Physics, 191, 82 (1989).

[17] Ph. Droz-Vincent, Reduction of Relativistic Three-Body Kinematics, hep-th/9905119, May 17,1999

[18] P. Van Alstine and H.W. Crater, J. Math. Phys. 23, 1997 (1982) H. W. Crater and P. Van Alstine, Ann. Phys. (N.Y.) 148, 57 (1983).

[19] H. Sazdjian, Phys. Rev. D1 33, 3401(1986), derives compatible two-body Dirac equations but from a different starting point without the use of supersymmetry. See also H. Sazdjian, Phys. Rev. D1 33, 3425 (1986).

[20] F.A. Berezin and M. S. Marinov, JETP Lett.21, 678 (1975), Ann. Phys. (N.Y.) 104, 336 (1977).

[21] C.A.P. Galvao and C. Teitelboim, J. Math. Phys. 21, 1863 (1980). See also A. Barducci, R. Casalbuoni and L. Lusanna, Nuovo Cimento A32,377 (1976).

[22] H. W. Crater and P. Van Alstine, Phys. Rev. Lett. 53, 1577 (1984).

[23] H. W. Crater and P. Van Alstine, Phys. Rev. D36, 3007 (1987).

[24] N. Nakanishi, Suppl. Prog. Theor. Phys.43 1, (1969)

[25] H. W. Crater, R. Becker, C. Y. Wong and P. Van Alstine, Phys. Rev. D1 46, 5117 (1992).

[26] I. T. Todorov, Phys. Rev. D3, 2351, 1971

[27] H. Jallouli and H. Sazdjian, Annals of Physics, 253, 376 (1997).

[28] H.W. Crater and D. Yang, J. Math. Phys. 32 2374, (1991).

[29] Our version of the constraint formalism fixes the relative values of these two parameters in 
terms of the invariant center of momentum energy. These fixed invariants are not free unlike the analogous "center of mass" parameter $\eta$ that often appears in the Bethe-Salpeter bound state formalism. In that formalism $\eta$ is only restricted to lie between 0 and 1 (although in QED is it often taken to be $\left.m_{i} /\left(m_{1}+m_{2}\right)\right)$. It is disconcerting that certain approaches that adopt this formalism use $\eta$ as a parameter in their spectral fits. We refer in particular to the work of P. Jain and H. Munczek, Phys. Rev. D 48, 5403 (1993) which combines the Bethe-Salpeter bound state formalism with that of the Schwinger-Dyson equation.

[30] H. W. Crater and P. Van Alstine, Phys. Rev. D30, 2585 (1984).

[31] H. W. Crater and P. Van Alstine, Phys. Rev. D46 476, (1992).

[32] H. Sazdjian, Phys. Lett.156B, 381 (1985).

[33] See H. Sazdjian, Proceedings of the International Symposium on Extended Objects and Bound Systems, Kairuzawa, Japan, (1992), pp 117-130 and [27] for a more recent treatment.

[34] H. W. Crater and P. Van Alstine, Phys. Rev. D 37, 1982 (1988)

[35] P. Van Alstine and H. W. Crater, Phys. Rev. D 34, 1932 (1986).

[36] A. Eddington, Proc. Roy. Soc. A122, 358 (1929); J.A. Gaunt, Phil. Trans. Roy. Soc, Vol 228, 151, (1929);Proc. Roy. Soc. A122, 153 (1929).

[37] P. Van Alstine, H. W. Crater Found. of Phys., 27 ,67 (1997).

[38] B.L. Aneva, J.I. Krapchev, and V.A. Rizov, Bulg. J. Phys 2,409, (1975). V.A. Rizov and I.T. Todorov, Fiz. Elem. Chastits At. Yadre 6,669 (1975) [translated in Sov. Jl. Part. Nucl. 6. 269 (1975)].

[39] G. Bohnert, R. Decker, A. Hornberg, H. Pilkuhn, H.G. Schlaile, Z. Phys. 2, 23 (1986)).

[40] J. Schwinger, Particles, Sources, and Fields (Addison-Wesley, Reading, 1973), Vol. 2, pp.348349.

[41] P.G. Bergmann, Rev. Mod. Phys. 33, 510 (1961)

[42] M.H.L. Pryce, Proc. Roy. Soc. (London) A195,6 (1948).

[43] T.D. Newton and E.P. Wigner, Rev. Mod. Phys. 21, 400 (1949).

[44] A.J. Hanson and T. Regge, Ann. Phys. (N.Y.) 87, 498 (1974)

[45] H. W. Crater and P. Van Alstine, Found. Of Phys. 244, 297 (1994).

[46] G. Morpurgo, Phys. Rev. D1, 41, 2865 (1990) Dillon and Morpurgo

[47] S. L. Adler and T. Piran, Phys. Lett., 117B, 91 (1982) and references contained therein.

[48] A. Gara, B. Durand, and L. Durand, Phys. Rev D1 40,843 (1989), 42, 1651 (1990). 
[49] A.J. Sommerer, J.R. Spence and J.P. Vary, Mod. Phys. Lett. A8, 3537 (1994), A.J. Sommerer, J.R. Spence and J.P. Vary, Phys.Rev C, 49, 513 (1994), A.J. Sommerer et al, Phys. Lett. B348, 277 (1995).

[50] D.D. Brayshaw, Phys. Rev. D 36, 1465 (1987)

[51] S. Godfrey and N. Isgur, Phys. Rev. D1 32, 189 (1985)

[52] P. A. M. Dirac, Proc. R. Soc. London A 117, 610 (1928).

[53] P. Long and H. W. Crater, J. Math. Phys. 39, 124 (1998)

[54] J.Mourad and H. Sazdjian, Journal of Physics G, 21, 267 (1995).

[55] Mourad and Sazdjian show that the covariant constraint approach in QED is free order by order from the spurious infrared singularities that force other approaches to abandon the manifestly covariant Feynman gauge in favor of the noncovariant Coulomb gauge. See also H. Jallouli and H. Sazdjian, J. Math. Phys. 38 4951, (1997)

[56] For positronium our results agree with the standard results in E.E. Salpeter, Phys. Rev. 87 328,(1952) and M. A. Stroscio, Phys. Rep. C22, 215(1975) without the annihilation diagram. Stroscio also gives the spectrum for the general hydrogenic (unequal mass) case but in the $j-j$ coupling scheme, rather than the $L S$ coupling scheme present in [25] here. The results for the $L S$ scheme is given in [38] but with a spectrum that does not have the correct $j=l$ spin mixing fine-structure even though the weak potential bound state equations given there are correct. The corrected spectrum appears in [25] and in J. Connell, Phys. Rev. D43, 1393, (1991).

[57] Review of Particle Physics, D. E. Groom et al., The European Physical Journal C15 1 (2000)

[58] The Babar Collabotation: B. Aubert et al, Phys.Rev.Lett. 90 242001,(2003)

[59] S. Ishida, M. Ishida, and T. Maeda, Prog. Theo. Phys. 104, 785 (2000)

[60] B Liu and H. Crater, Phys. Rev C 67024001 (2003)

[61] H. Crater, J. Comp. Phys. 115, 470 (1994)

[62] H. W. Crater, and P. Van Alstine, J. Math. Phys.31, 1998 (1990).

[63] All this extra structure makes possible straight-forward nonperturbative solution of the constraint equations and may account for the differences between the resulting (correct fieldtheoretic) spectrum and the incorrect spectrum produced by nonperturbative solution of the usual Breit equation for QED [37].

[64] H. Crater and P. Van Alstine, Phys. Lett., 100B, 166 (1981) 
[65] R.J. Yaes, Phys D 3, 3086 (1971)

[66] The value of that binding energy to this order is $w-2 m=m\left(-\alpha^{2} / 4-21 \alpha^{4} / 64\right)$.

[67] The results presented in this version of the Iowa State model are significantly improved over those produced by a different earlier version of the model, in which the Iowa State Group used the Salpeter equation with included couplings between positive and negative energy states, and an additional Breit interaction, J.R. Spence and J.P. Vary, Phys. Rev. C47, 1282 (1993). A significant part of the improvement may be due to alterations in the later paper [49] in the short distance part of the QCD potential, including a running coupling constant. The AdlerPiran potential that we use also includes a running coupling constant, but in configuration space instead of momentum space.

[68] We point out that our analytic singlet positronium result for the ground state is the closedform Sommerfeld-like result $[35] w=m \sqrt{2+2 / \sqrt{1+\frac{\alpha^{2}}{\left(1+\sqrt{\frac{1}{4}-\alpha^{2}}-\frac{1}{2}\right)^{2}}}} \doteq m\left(2-\frac{\alpha^{2}}{4}-\frac{21 \alpha^{4}}{64}\right)$ whose precise agreement with the perturbative results supports this reasoning.

[69] Note, however, that as long as $w$ is positive, our constituent c.m. energies are fixed to be positive by the constraints themselves (see [60]).

[70] D.B. Lichtenberg et al, Z. Phys. C19, 19 (1983)

[71] D.P. Stanley and D. Robson, D21 31 (1980)

[72] J.R. Spence and J.P. Vary, Phys. Lett B254, 1 ,(1991)

[73] Others on this relatively short list include [71] and P.C. Termeijer and J.A. Tjon, Phys. Rev C 49, 494 (1994). The latter authors use quasipotential equations in configuration space, including the Blankenbecler-Sugar equation and the equation of Mandelzweig and Wallace. Like ours, their equations contain the full Dirac structure of "positive and negative" energy states. We point out, however, that according to the work in [49], both of these quasipotential reductions fail to reproduce numerically even the ground state of positronium correctly in contrast to the work of $[25,35]$ and [49].

[74] A. Martin, Phys. Lett. B100, 511 (1981)

[75] A. Martin, Phys. Lett. B214, 561 (1988)

[76] G. Jazcko and L. Durand, Phys. Rev. D 58 1998, 114017-1,114017-9

[77] W.E. Caswell and G.P. Lepage, Phys. Rev. A18, 863 (1977) 


\section{TABLE I - MESON MASSES FROM COVARIANT CONSTRAINT DY- NAMICS}

NAME EXP. THEORY

$\begin{array}{lrr}\Upsilon: b \bar{b} 1^{3} S_{1} & 9.460(0.2) & 9.453(0.6) \\ \Upsilon: b \bar{b} 1^{3} P_{0} & 9.860(1.3) & 9.842(1.4) \\ \Upsilon: b \bar{b} 1^{3} P_{1} & 9.892(0.7) & 9.889(0.1) \\ \Upsilon: b \bar{b} 1^{3} P_{2} & 9.913(0.6) & 9.921(0.5) \\ \Upsilon: b \bar{b} 2^{3} S_{1} & 10.023(0.3) & 10.022(0.0) \\ \Upsilon: b \bar{b} 2^{3} P_{0} & 10.232(0.6) & 10.227(0.2) \\ \Upsilon: b \bar{b} 2^{3} P_{1} & 10.255(0.5) & 10.257(0.0) \\ \Upsilon: b \bar{b} 2^{3} P_{2} & 10.269(0.4) & 10.277(0.8) \\ \Upsilon: b \bar{b} 3^{3} S_{1} & 10.355(0.5) & 10.359(0.1) \\ \Upsilon: b \bar{b} 4^{3} S_{1} & 10.580(3.5) & 10.614(0.9) \\ \Upsilon: b \bar{b} 5^{3} S_{1} & 10.865(8.0) & 10.826(0.2) \\ \Upsilon: b \bar{b} 6^{3} S_{1} & 11.019(8.0) & 11.013(0.0) \\ B: b \bar{u} 1^{1} S_{0} & 5.279(1.8) & 5.273(0.1) \\ B: b \bar{d} 1^{1} S_{0} & 5.279(1.8) & 5.274(0.1) \\ B^{*}: b \bar{u} 1^{3} S_{1} & 5.325(1.8) & 5.321(0.1) \\ B_{s}: b \bar{s} 1^{1} S_{0} & 5.369(2.0) & 5.368(0.0) \\ B_{s}: b \bar{s} 1^{3} S_{1} & 5.416(3.3) & 5.427(0.1) \\ \eta_{c}: c \bar{c} 1^{1} S_{0} & 2.980(2.1) & 2.978(0.0) \\ \psi: c \bar{c} 1^{3} S_{1} & 3.097(0.0) & 3.129(12.6) \\ \chi_{0}: c \bar{c} 1^{1} P_{1} & 3.526(0.2) & 3.520(0.4) \\ \chi_{0}: c \bar{c} 1^{3} P_{0} & 3.415(1.0) & 3.407(0.4) \\ \chi_{1}: c \bar{c} 1^{3} P_{1} & 3.510(0.1) & 3.507(0.2) \\ \chi_{2}: c \bar{c} 1^{3} P_{2} & 3.556(0.1) & 3.549(0.6) \\ \eta_{c}: c \bar{c} 2^{1} S_{0} & 3.594(5.0) & 3.610(0.1) \\ \psi: c \bar{c} 2^{3} S_{1} & 3.686(0.1) & 3.688(0.1) \\ \psi: c \bar{c} 1^{3} D_{1} & 3.770(2.5) & 3.808(2.0) \\ \psi: c \bar{c} 3^{3} S_{1} & 4.040(10.0) & 4.081(0.2) \\ \psi: c \bar{c} 2^{3} D_{1} & 4.159(20.0) & 4.157(0.0) \\ & & \end{array}$




$$
\begin{aligned}
& \psi: c \bar{c} 3^{3} D_{1} \\
& 4.415(6.0) \quad 4.454(0.4) \\
& D: c \bar{u} 1^{1} S_{0} \quad 1.865(0.5) \quad 1.866(0.0) \\
& D: c \bar{d} 1^{1} S_{0} \quad 1.869(0.5) \quad 1.873(0.1) \\
& D^{*}: c \bar{u} 1^{3} S_{1} \quad 2.007(0.5) \quad 2.000(0.4) \\
& D^{*}: c \bar{d} 1^{3} S_{1} \quad 2.010(0.5) \quad 2.005(0.3) \\
& \begin{array}{lll}
D^{*}: c \bar{u} 1^{3} P_{1} & 2.422(1.8) & 2.407(0.6)
\end{array} \\
& D^{*}: c \bar{d} 1^{3} P_{1} \quad 2.428(1.8) \quad 2.411(0.5) \\
& D^{*}: c \bar{u} 1^{3} P_{2} \quad 2.459(2.0) \quad 2.382(11.3) \\
& D^{*}: c \bar{d} 1^{3} P_{2} \quad 2.459(4.0) \quad 2.386(3.5) \\
& D_{s}: c \bar{s} 1^{1} S_{0} \quad 1.968(0.6) \quad 1.976(0.5) \\
& D_{s}^{*}: c \bar{s} 1^{3} S_{1} \quad 2.112(0.7) \quad 2.123(0.9) \\
& D_{s}^{*}: c \bar{s} 1^{3} P_{1} \quad 2.535(0.3) \quad 2.511(6.2) \\
& D_{s}^{*}: c \bar{s} 1^{3} P_{2} \quad 2.574(1.7) \quad 2.514(9.6) \\
& K: s \bar{u} 1^{1} S_{0} \quad 0.494(0.0) \quad 0.492(0.0) \\
& K: s \bar{d} 1^{1} S_{0} \quad 0.498(0.0) \quad 0.492(0.4) \\
& K^{*}: s \bar{u} 1^{3} S_{1} \quad 0.892(0.2) \quad 0.910(0.6) \\
& K^{*}: s \bar{d} 1^{3} S_{1} \quad 0.896(0.3) \quad 0.910(0.3) \\
& K_{1}: s \bar{u} 1^{1} P_{1} \quad 1.273(7.0) \quad 1.408(3.2) \\
& K_{0}^{*}: s \bar{u} 1^{3} P_{0} \quad 1.429(4.0) \quad 1.314(0.7) \\
& K_{1}: s \bar{u} 1^{3} P_{1} \quad 1.402(7.0) \quad 1.506(1.0) \\
& K_{2}^{*}: s \bar{u} 1^{3} P_{2} \quad 1.425(1.3) \quad 1.394(0.5) \\
& K_{2}^{*}: s \bar{d} 1^{3} P_{2} \quad 1.432(1.3) \quad 1.394(0.6) \\
& K^{*}: s \bar{u} 2^{1} S_{0} \quad 1.460(30.0) \quad 1.591(0.2) \\
& K^{*}: s \bar{u} 2^{3} S_{1} \quad 1.412(12.0) \quad 1.800(6.7) \\
& K_{2}: s \bar{u} 1^{1} D_{2} \quad 1.773(8.0) \quad 1.877(0.8) \\
& K^{*}: s \bar{u} 1^{3} D_{1} \quad 1.714(20.0) \quad 1.985(1.4) \\
& K_{2}: s \bar{u} 1^{3} D_{2} \quad 1.816(10.0) \quad 1.945(1.3) \\
& K_{3}: s \bar{u} 1^{3} D_{3} \quad 1.770(10.0) \quad 1.768(0.0) \\
& K^{*}: s \bar{u} 3^{1} S_{0} \quad 1.830(30.0) \quad 2.183(1.4) \\
& K_{2}^{*}: s \bar{u} 2^{3} P_{2} \quad 1.975(22.0) \quad 2.098(0.2) \\
& K_{4}^{*}: s \bar{u} 1^{3} F_{4} \quad 2.045(9.0) \quad 2.078(0.1) \\
& K_{2}: s \bar{u} 2^{3} D_{2} \quad 2.247(17.0) \quad 2.373(0.5)
\end{aligned}
$$




\begin{tabular}{|c|c|c|}
\hline$K_{5}^{*}: s \bar{u} 1^{3} G_{5}$ & $2.382(33.0)$ & $2.344(0.0)$ \\
\hline$K_{3}^{*}: s \bar{u} 2^{3} F_{3}$ & $2.324(24.0)$ & $2.636(1.9)$ \\
\hline$K_{4}^{*}: s \bar{u} 2^{3} F_{4}$ & $2.490(20.0)$ & $2.757(1.6)$ \\
\hline$\phi: s \bar{s} 1^{3} S_{1}$ & $1.019(0.0)$ & $1.033(2.2)$ \\
\hline$f_{0}: s \bar{s} 1^{3} P_{0}$ & $1.370(40.0)$ & $1.319(0.0)$ \\
\hline$f_{1}: s \bar{s} 1^{3} P_{1}$ & $1.512(4.0)$ & $1.533(0.3)$ \\
\hline$f_{2}: s \bar{s} 1^{3} P_{2}$ & $1.525(5.0)$ & $1.493(0.3)$ \\
\hline$\phi: s \bar{s} 2^{3} S_{1}$ & $1.680(20.0)$ & $1.850(0.8)$ \\
\hline$\phi: s \bar{s} 1^{3} D_{3}$ & $1.854(7.0)$ & $1.848(0.0)$ \\
\hline$f_{2}: s \bar{s} 2^{3} P_{2}$ & $2.011(69.0)$ & $2.160(0.1)$ \\
\hline$f_{2}: s \bar{s} 3^{3} P_{2}$ & $2.297(28.0)$ & $2.629(1.6)$ \\
\hline$\pi: u \bar{d} 1^{1} S_{0}$ & $0.140(0.0)$ & $0.144(0.2)$ \\
\hline$\rho: u \bar{d} 1^{3} S_{1}$ & $0.767(1.2)$ & $0.792(0.1)$ \\
\hline$b_{1}: u \bar{d} 1^{1} P_{1}$ & $1.231(10.0)$ & $1.392(2.1)$ \\
\hline$a_{0}: u \bar{d} 1^{3} P_{0}$ & $1.450(40.0)$ & $1.491(0.0)$ \\
\hline$a_{1}: u \bar{d} 1^{3} P_{1}$ & $1.230(40.0)$ & $1.568(0.7)$ \\
\hline$a_{2}: u \bar{d} 1^{3} P_{2}$ & $1.318(0.7)$ & $1.310(0.0)$ \\
\hline$\pi: u \bar{d} 2^{1} S_{0}$ & $1.300(100.0)$ & $1.536(0.1)$ \\
\hline$\rho: u \bar{d} 2^{3} S_{1}$ & $1.465(25.0)$ & $1.775(1.4)$ \\
\hline$\pi_{2}: u \bar{d} 1^{1} D_{2}$ & $1.670(20.0)$ & $1.870(0.9)$ \\
\hline$\rho: u \bar{d} 1^{3} D_{1}$ & $1.700(20.0)$ & $1.986(1.9)$ \\
\hline$\rho_{3}: u \bar{d} 1^{3} D_{3}$ & $1.691(5.0)$ & $1.710(0.0)$ \\
\hline$\pi: u \bar{d} 3^{1} S_{0}$ & $1.795(10.0)$ & $2.166(7.9)$ \\
\hline$\rho: u \bar{d} 3^{3} S_{1}$ & $2.149(17.0)$ & $2.333(0.7)$ \\
\hline$\rho_{4}: u \bar{d} 1^{3} F_{4}$ & $2.037(26.0)$ & $2.033(0.0)$ \\
\hline$\pi_{2}: u \bar{d} 2^{1} D_{2}$ & $2.090(29.0)$ & $2.367(0.5)$ \\
\hline$\rho_{3}: u \bar{d} 2^{3} D_{3}$ & $2.250(45.0)$ & $2.305(0.0)$ \\
\hline$\rho_{5}: u \bar{d} 1^{3} G_{5}$ & $2.330(35.0)$ & $2.307(0.0)$ \\
\hline$\rho_{6}: u \bar{d} 1^{3} H_{6}$ & $2.450(130.0)$ & $2.547(0.0)$ \\
\hline 2 & 0.0 & 101.0 \\
\hline
\end{tabular}


TABLE II COMPARISON OF MESON MASSES FROM

WISCONSIN MODEL II and COVARIANT CONSTRAINT DYNAMICS

NAME

EXP.

WISC2

CCD

\begin{tabular}{|c|c|c|c|}
\hline$\Upsilon: b \bar{b} 1^{3} S_{1}$ & $9.460(0.2)$ & $9.426(62.6)$ & $9.454(2.0)$ \\
\hline$\Upsilon: b \bar{b} 1^{3} P_{0}$ & $9.860(1.3)$ & $9.862(0.1)$ & $9.845(4.5)$ \\
\hline$\Upsilon: b \bar{b} 1^{3} P_{1}$ & $9.892(0.7)$ & $9.892(0.0)$ & $9.890(0.1)$ \\
\hline$\Upsilon: b \bar{b} 1^{3} P_{2}$ & $9.913(0.6)$ & $9.917(0.7)$ & $9.919(1.6)$ \\
\hline$\Upsilon: b \bar{b} 2^{3} S_{1}$ & $10.023(0.3)$ & $10.028(1.3)$ & $10.024(0.1)$ \\
\hline$\Upsilon: b \bar{b} 2^{3} P_{0}$ & $10.232(1.1)$ & $10.238(1.5)$ & $10.229(0.4)$ \\
\hline$\Upsilon: b \bar{b} 2^{3} P_{1}$ & $10.255(0.6)$ & $10.256(0.0)$ & $10.257(0.2)$ \\
\hline$\Upsilon: b \bar{b} 2^{3} P_{2}$ & $10.268(0.6)$ & $10.270(0.2)$ & $10.276(3.1)$ \\
\hline$: b \bar{b} 3^{3} S_{1}$ & $10.355(0.5)$ & $10.359(0.7)$ & $10.359(0.7)$ \\
\hline$B: b \bar{d} 1^{1} S_{0}$ & $5.279(2.1)$ & $5.381(137.2)$ & $5.274(0.3)$ \\
\hline$\eta_{c}: c \bar{c} 1^{1} S_{0}$ & $2.979(1.9)$ & $2.967(1.4)$ & $2.975(0.1)$ \\
\hline$\psi: c \bar{c} 1^{3} S_{1}$ & $3.097(0.1)$ & $3.167(272.4)$ & $3.120(28.8)$ \\
\hline$\chi_{0}: c \bar{c} 1^{3} P_{0}$ & $3.415(1.0)$ & $3.402(5.1)$ & $3.412(0.2)$ \\
\hline$\chi_{1}: c \bar{c} 1^{3} P_{1}$ & $3.510(0.1)$ & $3.493(17.5)$ & $3.505(1.8)$ \\
\hline$\chi_{2}: c \bar{c} 1^{3} P_{2}$ & $3.556(0.1)$ & $3.548(4.0)$ & $3.538(18.1)$ \\
\hline$\eta_{c}: c \bar{c} 2^{1} S_{0}$ & $3.594(5.0)$ & $3.621(1.5)$ & $3.611(0.6)$ \\
\hline$\psi: c \bar{c} 2^{3} S_{1}$ & $3.686(0.1)$ & $3.668(17.9)$ & $3.688(0.3)$ \\
\hline$D: c \bar{d} 1^{1} S_{0}$ & $1.869(0.5)$ & $1.983(574.6)$ & $1.875(1.5)$ \\
\hline$D^{*}: c \bar{d} 1^{3} S_{1}$ & $2.010(0.6)$ & $2.010(0.0)$ & $2.003(1.9)$ \\
\hline$D_{s}: c \bar{s} 1^{1} S_{0}$ & $1.969(0.7)$ & 2.097( 671.1) & $1.968(0.1)$ \\
\hline$D_{s}^{*}: c \bar{s} 1^{3} S_{1}$ & $2.110(2.0)$ & $2.148(52.7)$ & $2.106(0.6)$ \\
\hline$K: s \bar{d} 1^{1} S_{0}$ & $0.498(0.0)$ & $0.743(3340.4)$ & $0.498(0.0)$ \\
\hline$K^{*}: s \bar{d} 1^{3} S_{1}$ & $0.896(0.3)$ & $0.870(5.1)$ & $0.918(3.5)$ \\
\hline$\phi: s \bar{s} 1^{3} S_{1}$ & $1.019(0.0)$ & $1.019(0.0)$ & $1.020(0.0)$ \\
\hline$\phi: s \bar{s} 2^{3} S_{1}$ & $1.680(50.0)$ & $1.510(0.9)$ & $1.424(2.1)$ \\
\hline & 0.0 & 5168.9 & 72.8 \\
\hline
\end{tabular}




\section{TABLE III -COMPARISON OF MESON MASSES FROM}

\section{SPENCE-VARY MODEL and COVARIANT CONSTRAINT DYNAMICS}

NAME

EXP. SPENCE \& VARY

CCD

\begin{tabular}{|c|c|c|c|}
\hline$\Upsilon: b \bar{b} 1^{3} S_{1}$ & $9.460(9.460)$ & $9.452(-8)$ & $9.444(-16)$ \\
\hline$\Upsilon: b \bar{b} 1^{3} P_{0}$ & $9.860(9.860)$ & $9.843(-17)$ & $9.836(-24)$ \\
\hline$\Upsilon: b \bar{b} 1^{3} P_{1}$ & $9.892(9.893)$ & $9.863(-29)$ & $9.886(-7)$ \\
\hline$\Upsilon: b \bar{b} 1^{3} P_{2}$ & $9.913(9.913)$ & $9.928(+15)$ & $9.921(+8)$ \\
\hline$\Upsilon: b \bar{b} 2^{3} S_{1}$ & $10.023(10.023)$ & $9.996(-27)$ & $10.022(+1)$ \\
\hline$\Upsilon: b \bar{b} 2^{3} P_{0}$ & $10.232(10.232)$ & $10.198(-34)$ & $10.230(+2)$ \\
\hline$\Upsilon: b \bar{b} 2^{3} P_{1}$ & $10.255(10.255)$ & $10.214(-41)$ & $10.261(+6)$ \\
\hline$\Upsilon: b \bar{b} 2^{3} P_{2}$ & $10.268(19.269)$ & $10.270(+2)$ & $10.284(+17)$ \\
\hline$\Upsilon: b \bar{b} 3^{3} S_{1}$ & $10.355(10.355)$ & $10.331(-24)$ & $10.367(+12)$ \\
\hline$\Upsilon: b \bar{b} 4^{3} S_{1}$ & $10.580(10.580)$ & $10.611(+31)$ & $10.627(+47)$ \\
\hline$\Upsilon: b \bar{b} 5^{3} S_{1}$ & $10.865(10.865)$ & $10.860(-5)$ & $10.645(-20)$ \\
\hline$\Upsilon: b \bar{b} 6^{3} S_{1}$ & $11.019(11.019)$ & $11.086(+67)$ & $11.036(17)$ \\
\hline$B: b \bar{u} 1^{1} S_{0}$ & $5.271(5.279)$ & $5.342(+63)$ & $5.267(-12)$ \\
\hline$B^{*}: b \bar{u} 1^{3} S_{1}$ & $5.352(5.325)$ & $5.347(-5)$ & $5.317(-8)$ \\
\hline$\eta_{c}: c \bar{c} 1^{1} S_{0}$ & $2.979(2.980)$ & $2.993(+14)$ & $2.969(-11)$ \\
\hline$\psi: c \bar{c} 1^{3} S_{1}$ & $3.097(3.097)$ & $3.091(-6)$ & $3.128(+31)$ \\
\hline$\chi_{0}: c \bar{c} 1^{1} P_{1}$ & $3.526(3.526)$ & $3.471(-55)$ & $3.520(-6)$ \\
\hline$\chi_{0}: c \bar{c} 1^{3} P_{0}$ & $3.415(3.415)$ & $3.383(-32)$ & $3.396(-19)$ \\
\hline$\chi_{1}: c \bar{c} 1^{3} P_{1}$ & $3.511(3.511)$ & $3.461(-50)$ & $3.504(-7)$ \\
\hline$\chi_{2}: c \bar{c} 1^{3} P_{2}$ & $3.556(3.556)$ & $3.556(0)$ & $3.555(-1)$ \\
\hline$\eta_{c}: c \bar{c} 2^{1} S_{0}$ & $3.594(3.594)$ & $3.640(+46)$ & $3.606(+12)$ \\
\hline$\psi: c \bar{c} 2^{3} S_{1}$ & $3.686(3.686)$ & $3.688(+2)$ & $3.688(+2)$ \\
\hline$\psi: c \bar{c} 1^{3} D_{1}$ & $3.770(3.770)$ & $3.741(-29)$ & $3.806(+36)$ \\
\hline$\psi: c \bar{c} 3^{3} S_{1}$ & $4.040(4.040)$ & $4.104(+64)$ & $4.083(+43)$ \\
\hline$\psi: c \bar{c} 2^{3} D_{1}$ & $4.159(4.159)$ & $4.136(-23)$ & $4.161(+2)$ \\
\hline$\psi: c \bar{c} 3^{3} D_{1}$ & $4.415(4.415)$ & $4.456(+41)$ & $4.462(+47)$ \\
\hline$D: c \bar{u} 1^{1} S_{0}$ & $1.865(1.8645)$ & $1.897(+32)$ & $1.854(-10)$ \\
\hline$D^{*}: c \bar{u} 1^{3} S_{1}$ & $2.007(2.007)$ & $2.004(-3)$ & $1.991(-16)$ \\
\hline
\end{tabular}




$\begin{array}{lrrr}D^{*}: c \bar{u} 1^{3} P_{1} & 2.420(2.422) & 2.358(-72) & 2.373(-47) \\ D_{s}: c \bar{s} 1^{1} S_{0} & 1.971(1.969) & 1.968(-3) & 1.981(+12) \\ D_{s}^{*}: c \bar{s} 1^{3} S_{1} & 2.110(2.112) & 2.076(-34) & 2.137(+25) \\ K: s \bar{u} 1^{1} S_{0} & 0.494(0.494) & 0.495(+1) & 0.511(+17) \\ K^{*}: s \bar{u} 1^{3} S_{1} & 0.892(0.892) & 0.916(+24) & 0.887(-5) \\ K_{1}: s \bar{u} 1^{1} P_{1} & 1.270(1.273) & 1.287(+17) & 1.327(+57) \\ K_{1}^{*}: s \bar{u} 1^{3} P_{1} & 1.406(1.402) & 1.330(-76) & 1.405(+3) \\ K_{2}^{*}: s \bar{u} 1^{3} P_{2} & 1.426(1.426) & 1.330(-96) & 1.348(-78) \\ K_{2}: s \bar{u} 1^{1} D_{2} & 1.770(1.776) & 1.633(-137) & 1.709(-85) \\ \phi: s \bar{s} 1^{3} S_{1} & 1.019(1.019) & 1.020(+1) & 1.048(+29) \\ f_{2}: s \bar{s} 1^{3} P_{2} & 1.525(1.525) & 1.526(+1) & 1.488(-37) \\ \phi: s \bar{s} 2^{3} S_{1} & 1.680(1.680) & 1.645(-35) & 1.803(+123) \\ \pi: u \bar{d} 1^{1} S_{0} & 0.140(0.140) & 0.135(-5) & 0.143(+3) \\ \rho: u \bar{d} 1^{3} S_{1} & 0.768(0.769) & 0.812(+44) & 0.736(-33) \\ b_{1}: u \bar{d} 1^{1} P_{1} & 1.232(1.230) & 1.219(-13) & 1.255(+25) \\ a_{1}: u \bar{d} 1^{3} P_{1} & 1.260(1.230) & 1.223(-37) & 1.534(+185) \\ a_{2}: u \bar{d} 1^{3} P_{2} & 1.318(1.318) & 1.367(+49) & 1.223(-95) \\ \pi: u \bar{d} 2^{1} S_{0} & 1.300(1.300) & 1.439(+139) & 1.474(174) \\ \pi_{2}: u \bar{d} 1^{1} D_{2} & 1.670(1.670) & 515(-155) & 1.780(+110) \\ R M S & & & 53\end{array}$




\section{TABLE IV - COMPARISON OF MESON MASSES FROM}

\section{BRAYSHAW MODEL and COVARIANT CONSTRAINT DYNAMICS}

NAME

EXP. BRAYSHAW

CCD

\begin{tabular}{|c|c|c|c|}
\hline$\Upsilon: b \bar{b} 1^{3} S_{1}$ & $9.460(0.2)$ & $9.452(1.3)$ & $9.451(1.7)$ \\
\hline$\Upsilon: b \bar{b} 1^{3} P_{0}$ & $9.860(1.3)$ & $9.866(0.3)$ & $9.842(2.5)$ \\
\hline$\Upsilon: b \bar{b} 1^{3} P_{1}$ & $9.892(0.7)$ & $9.910(4.5)$ & $9.889(0.1)$ \\
\hline$\Upsilon: b \bar{b} 1^{3} P_{2}$ & $9.913(0.6)$ & $9.926(2.5)$ & $9.920(0.7)$ \\
\hline$\Upsilon: b \bar{b} 2^{3} S_{1}$ & $10.023(0.3)$ & $10.007(4.8)$ & $10.023(0.0)$ \\
\hline$\Upsilon: b \bar{b} 2^{3} P_{0}$ & $10.232(0.6)$ & $10.214(4.9)$ & $10.229(0.1)$ \\
\hline$\Upsilon: b \bar{b} 2^{3} P_{1}$ & $10.255(0.5)$ & $10.252(0.1)$ & $10.258(0.1)$ \\
\hline$\Upsilon: b \bar{b} 2^{3} P_{2}$ & $10.268(0.4)$ & $10.265(0.2)$ & $10.278(1.8)$ \\
\hline$\Upsilon: b \bar{b} 3^{3} S_{1}$ & $10.355(0.5)$ & $10.342(2.8)$ & $10.360(0.4)$ \\
\hline$\Upsilon: b \bar{b} 4^{3} S_{1}$ & $10.580(3.5)$ & $10.662(9.4)$ & $10.617(1.9)$ \\
\hline$B: b \bar{u} 1^{1} S_{0}$ & $5.279(1.8)$ & $5.332(13.7)$ & $5.270(0.3)$ \\
\hline$B^{*}: b \bar{u} 1^{3} S_{1}$ & $5.325(1.8)$ & $5.377(13.2)$ & $5.317(0.3)$ \\
\hline$\eta_{c}: c \bar{c} 1^{1} S_{0}$ & $2.980(2.1)$ & $3.011(3.5)$ & $2.976(0.0)$ \\
\hline$\psi: c \bar{c} 1^{3} S_{1}$ & $3.097(0.1)$ & $3.129(21.0)$ & $3.127(17.8)$ \\
\hline$\chi_{0}: c \bar{c} 1^{1} P_{1}$ & $3.524(0.2)$ & $3.498(13.0)$ & $3.520(0.3)$ \\
\hline$\chi_{0}: c \bar{c} 1^{3} P_{0}$ & $3.415(1.0)$ & $3.410(0.3)$ & $3.409(0.4)$ \\
\hline$\chi_{1}: c \bar{c} 1^{3} P_{1}$ & $3.510(0.1)$ & $3.514(0.2)$ & $3.508(0.2)$ \\
\hline$\chi_{2}: c \bar{c} 1^{3} P_{2}$ & $3.556(0.1)$ & $3.540(5.2)$ & $3.547(1.5)$ \\
\hline$\eta_{c}: c \bar{c} 2^{1} S_{0}$ & $3.594(5.0)$ & $3.580(0.2)$ & $3.612(0.3)$ \\
\hline$\psi: c \bar{c} 2^{3} S_{1}$ & $3.686(0.1)$ & $3.680(0.7)$ & $3.691(0.4)$ \\
\hline$\psi: c \bar{c} 1^{3} D_{1}$ & $3.770(2.5)$ & $3.773(0.0)$ & $3.811(4.0)$ \\
\hline$\psi: c \bar{c} 3^{3} S_{1}$ & $4.040(10.0)$ & $4.246(8.0)$ & $4.086(0.4)$ \\
\hline$\psi: c \bar{c} 2^{3} D_{1}$ & $4.159(20.0)$ & $4.288(0.8)$ & $4.163(0.0)$ \\
\hline$D: c \bar{u} 1^{1} S_{0}$ & $1.865(0.5)$ & $1.903(24.2)$ & $1.864(0.0)$ \\
\hline$D^{*}: c \bar{u} 1^{3} S_{1}$ & $2.007(1.4)$ & $2.046(24.5)$ & $1.997(1.7)$ \\
\hline$D^{*}: c \bar{u} 1^{3} P_{1}$ & $2.422(1.8)$ & $2.428(0.1)$ & $2.413(0.3)$ \\
\hline$D^{*}: c \bar{u} 1^{3} P_{2}$ & $2.459(2.0)$ & $2.458(0.0)$ & $2.383(18.8)$ \\
\hline$D_{s}: c \bar{s} 1^{1} S_{0}$ & $1.969(0.6)$ & $1.976(0.8)$ & $1.974(0.4)$ \\
\hline
\end{tabular}




\begin{tabular}{|c|c|c|c|}
\hline$D_{s}^{*}: c \bar{s} 1^{3} S_{1}$ & $2.112(2.0)$ & $2.134(6.6)$ & $2.119(0.7)$ \\
\hline$D_{s}^{*}: c \bar{s} 1^{3} P_{1}$ & $2.535(0.3)$ & $2.515(7.2)$ & $2.515(7.0)$ \\
\hline$D_{s}^{*}: c \bar{s} 1^{3} P_{2}$ & $2.574(1.7)$ & $2.546(3.6)$ & $2.513(17.0)$ \\
\hline$K: s \bar{u} 1^{1} S_{0}$ & $0.494(0.0)$ & $0.495(0.0)$ & $0.492(0.1)$ \\
\hline$K^{*}: s \bar{u} 1^{3} S_{1}$ & $0.892(0.2)$ & $0.905(0.5)$ & $0.908(0.7)$ \\
\hline$K_{1}: s \bar{u} 1^{1} P_{1}$ & $1.273(7.0)$ & $1.355(1.1)$ & $1.421(3.6)$ \\
\hline$K_{0}^{*}: s \bar{u} 1^{3} P_{0}$ & $1.430(4.0)$ & $1.086(10.8)$ & $1.349(0.6)$ \\
\hline$K_{1}: s \bar{u} 1^{3} P_{1}$ & $1.402(7.0)$ & $1.294(3.4)$ & $1.524(4.3)$ \\
\hline$K_{2}^{*}: s \bar{u} 1^{3} P_{2}$ & $1.425(1.3)$ & $1.409(0.2)$ & $1.399(0.5)$ \\
\hline$K^{*}: s \bar{u} 1^{3} D_{1}$ & $1.714(20.0)$ & $1.690(0.0)$ & $2.004(2.6)$ \\
\hline$K_{2}: s \bar{u} 1^{3} D_{2}$ & $1.816(10.0)$ & $1.764(0.4)$ & $1.892(0.8)$ \\
\hline$K_{3}: s \bar{u} 1^{3} D_{3}$ & $1.770(10.0)$ & $1.770(0.0)$ & $1.780(0.0)$ \\
\hline$\phi: s \bar{s} 1^{3} S_{1}$ & $1.019(0.0)$ & $1.022(0.1)$ & $1.030(2.1)$ \\
\hline$f_{0}: s \bar{s} 1^{3} P_{0}$ & $1.370(40.0)$ & $1.185(0.4)$ & $1.345(0.0)$ \\
\hline$f_{1}: s \bar{s} 1^{3} P_{1}$ & $1.512(4.0)$ & $1.446(4.5)$ & $1.546(1.2)$ \\
\hline$f_{2}: s \bar{s} 1^{3} P_{2}$ & $1.525(5.0)$ & $1.511(0.1)$ & $1.496(0.4)$ \\
\hline$\phi: s \bar{s} 2^{3} S_{1}$ & $1.680(20.0)$ & $1.778(0.4)$ & $1.860(1.4)$ \\
\hline$\phi: s \bar{s} 1^{3} D_{3}$ & $1.854(7.0)$ & $1.922(1.4)$ & $1.856(0.0)$ \\
\hline$\pi: u \bar{d} 1^{1} S_{0}$ & $0.140(0.0)$ & $0.140(0.0)$ & $0.143(0.2)$ \\
\hline$\rho: u \bar{d} 1^{3} S_{1}$ & $0.767(1.2)$ & $0.776(0.0)$ & $0.790(0.2)$ \\
\hline$b_{1}: u \bar{d} 1^{1} P_{1}$ & $1.231(10.0)$ & $1.202(0.1)$ & $1.411(4.4)$ \\
\hline$a_{0}: u \bar{d} 1^{3} P_{0}$ & $1.450(40.0)$ & $0.990(2.4)$ & $1.542(0.1)$ \\
\hline$a_{1}: u \bar{d} 1^{3} P_{1}$ & $1.230(40.0)$ & $1.253(0.0)$ & $1.590(1.3)$ \\
\hline$a_{2}: u \bar{d} 1^{3} P_{2}$ & $1.318(7.0)$ & $1.302(0.2)$ & $1.318(0.0)$ \\
\hline$\pi: u \bar{d} 2^{1} S_{0}$ & $1.300(100.0)$ & $1.028(0.1)$ & $1.543(0.1)$ \\
\hline$\pi_{2}: u \bar{d} 1^{1} D_{2}$ & $1.670(20.0)$ & $1.593(0.2)$ & $1.883(1.6)$ \\
\hline$\rho: u \bar{d} 1^{3} D_{1}$ & $1.700(20.0)$ & $1.741(0.1)$ & $1.998(3.4)$ \\
\hline$\rho_{3}: u \bar{d} 1^{3} D_{3}$ & $1.691(5.0)$ & $1.680(0.0)$ & $1.722(0.2)$ \\
\hline$\chi^{2}$ & 0.0 & 204.2 & 111.0 \\
\hline
\end{tabular}




\section{TABLE V - COMPARISON OF MESON MASSES FROM}

\section{ISGUR-WISE MODEL and COVARIANT CONSTRAINT DYNAMICS}

NAME

EXP. ISGUR\&WISE

CCD

$\begin{array}{lrrr}\Upsilon: b \bar{b} 1^{3} S_{1} & 9.460(0.2) & 9.460(0.0) & 9.453(0.8) \\ \Upsilon: b \bar{b} 1^{3} P_{0} & 9.860(1.3) & 9.850(0.5) & 9.842(1.6) \\ \Upsilon: b \bar{b} 1^{3} P_{1} & 9.892(0.7) & 9.880(1.4) & 9.889(0.1) \\ \Upsilon: b \bar{b} 1^{3} P_{2} & 9.913(0.6) & 9.900(1.8) & 9.921(0.6) \\ \Upsilon: b \bar{b} 2^{3} S_{1} & 10.023(0.3) & 10.000(6.9) & 10.023(0.0) \\ \Upsilon: b \bar{b} 2^{3} P_{0} & 10.232(0.6) & 10.230(0.0) & 10.228(0.2) \\ \Upsilon: b \bar{b} 2^{3} P_{1} & 10.255(0.5) & 10.250(0.3) & 10.257(0.0) \\ \Upsilon: b \bar{b} 2^{3} P_{2} & 10.269(0.4) & 10.260(1.0) & 10.277(0.8) \\ \Upsilon: b \bar{b} 3^{3} S_{1} & 10.355(0.5) & 10.350(0.3) & 10.359(0.2) \\ \Upsilon: b \bar{b} 4^{3} S_{1} & 10.580(3.5) & 10.630(2.4) & 10.615(1.2) \\ \Upsilon: b \bar{b} 5^{3} S_{1} & 10.865(8.0) & 10.880(0.0) & 10.828(0.2) \\ \Upsilon: b \bar{b} 6^{3} S_{1} & 11.019(8.0) & 11.100(1.2) & 11.014(0.0) \\ B: b \bar{u} 1^{1} S_{0} & 5.279(1.8) & 5.310(3.3) & 5.272(0.2) \\ B^{*}: b \bar{u} 1^{3} S_{1} & 5.325(1.8) & 5.370(6.9) & 5.319(0.1) \\ B_{s}: b \bar{s} 1^{1} S_{0} & 5.369(2.0) & 5.390(1.2) & 5.368(0.0) \\ B_{s}: b \bar{s} 1^{3} S_{1} & 5.416(3.3) & 5.450(1.4) & 5.426(0.1) \\ \eta_{c}: c \bar{c} 1^{1} S_{0} & 2.980(2.1) & 2.970(0.2) & 2.978(0.0) \\ \psi: c \bar{c} 1^{3} S_{1} & 3.097(0.0) & 3.100(0.1) & 3.128(14.1) \\ \chi_{0}: c \bar{c} 1^{1} P_{1} & 3.526(0.2) & 3.520(0.5) & 3.520(0.5) \\ \chi_{0}: c \bar{c} 1^{3} P_{0} & 3.415(1.0) & 3.440(4.4) & 3.408(0.4) \\ \chi_{1}: c \bar{c} 1^{3} P_{1} & 3.510(0.1) & 3.510(0.0) & 3.507(0.2) \\ \chi_{2}: c \bar{c} 1^{3} P_{2} & 3.556(0.1) & 3.550(0.5) & 3.548(0.9) \\ \eta_{c}: c \bar{c} 2^{1} S_{0} & 3.594(5.0) & 3.620(0.4) & 3.611(0.2) \\ \psi: c \bar{c} 2^{3} S_{1} & 3.686(0.1) & 3.680(0.5) & 3.689(0.1) \\ \left.\psi: c \bar{c} 1^{3} D_{1} D_{1}\right) & 3.770(2.5) & 3.820(4.2) & 3.809(2.5) \\ \psi & 4.040(10.0) & 4.100(0.5) & 4.082(0.2) \\ \left.{ }^{3} S_{1} D_{1}\right) & 4.190(0.0) & 4.159(0.0) \\ \Psi .159(20.0) & 4.450(0.4) & 4.456(0.6)\end{array}$




\begin{tabular}{|c|c|c|c|}
\hline$D: c \bar{u} 1^{1} S_{0}$ & $1.865(0.5)$ & $1.880(2.7)$ & $1.865(0.0)$ \\
\hline$D^{*}: c \bar{u} 1^{3} S_{1}$ & $2.007(0.5)$ & $2.040(12.6)$ & $1.998(0.8)$ \\
\hline$D^{*}: c \bar{u} 1^{3} P_{1}$ & $2.422(1.8)$ & $2.440(0.9)$ & $2.408(0.6)$ \\
\hline$D^{*}: c \bar{u} 1^{3} P_{2}$ & $2.459(2.0)$ & $2.500(3.8)$ & $2.381(13.6)$ \\
\hline$D_{s}: c \bar{s} 1^{1} S_{0}$ & $1.968(0.6)$ & $1.980(1.4)$ & $1.976(0.6)$ \\
\hline$D_{s}^{*}: c \bar{s} 1^{3} S_{1}$ & $2.112(0.7)$ & $2.130(3.0)$ & $2.121(0.8)$ \\
\hline$D_{s}^{*}: c \bar{s} 1^{3} P_{1}$ & $2.535(0.3)$ & $2.530(0.4)$ & $2.512(6.7)$ \\
\hline$D_{s}^{*}: c \bar{s} 1^{3} P_{2}$ & $2.574(1.7)$ & $2.590(0.9)$ & $2.513(11.6)$ \\
\hline$K: s \bar{u} 1^{1} S_{0}$ & $0.494(0.0)$ & $0.470(8.0)$ & $0.494(0.0)$ \\
\hline$K^{*}: s \bar{u} 1^{3} S_{1}$ & $0.892(0.2)$ & $0.900(0.1)$ & $0.907(0.5)$ \\
\hline$K_{1}: s \bar{u} 1^{1} P_{1}$ & $1.273(7.0)$ & $1.340(0.5)$ & $1.411(2.2)$ \\
\hline$K_{0}^{*}: s \bar{u} 1^{3} P_{0}$ & $1.429(4.0)$ & $1.240(2.3)$ & $1.323(0.7)$ \\
\hline$K_{1}: s \bar{u} 1^{3} P_{1}$ & $1.402(7.0)$ & $1.380(0.1)$ & $1.509(2.3)$ \\
\hline$K_{2}^{*}: s \bar{u} 1^{3} P_{2}$ & $1.425(1.3)$ & $1.430(0.0)$ & $1.393(0.5)$ \\
\hline$K^{*}: s \bar{u} 2^{1} S_{0}$ & $1.460(30.0)$ & $1.450(0.0)$ & $1.593(0.2)$ \\
\hline$K^{*}: s \bar{u} 2^{3} S_{1}$ & $1.412(12.0)$ & $1.580(1.5)$ & $1.801(7.9)$ \\
\hline$K_{2}: s \bar{u} 1^{1} D_{2}$ & $1.773(8.0)$ & $1.780(0.0)$ & $1.879(1.1)$ \\
\hline$K^{*}: s \bar{u} 1^{3} D_{1}$ & $1.714(20.0)$ & $1.780(0.1)$ & $1.988(1.6)$ \\
\hline$K_{2}: s \bar{u} 1^{3} D_{2}$ & $1.816(10.0)$ & $1.810(0.0)$ & $1.947(1.5)$ \\
\hline$K_{3}: s \bar{u} 1^{3} D_{3}$ & $1.770(10.0)$ & $1.790(0.0)$ & $1.770(0.0)$ \\
\hline$K^{*}: s \bar{u} 3^{1} S_{0}$ & $1.830(30.0)$ & $2.020(0.5)$ & $2.188(1.7)$ \\
\hline$K_{2}^{*}: s \bar{u} 2^{3} P_{2}$ & $1.975(22.0)$ & $1.940(0.0)$ & $2.098(0.3)$ \\
\hline$K_{4}^{*}: s \bar{u} 1^{3} F_{4}$ & $2.045(9.0)$ & $2.110(0.3)$ & $2.080(0.1)$ \\
\hline$K_{2}: s \bar{u} 2^{3} D_{2}$ & $2.247(17.0)$ & $2.260(0.0)$ & $2.377(0.7)$ \\
\hline$K_{5}^{*}: s \bar{u} 1^{3} G_{5}$ & $2.382(33.0)$ & $2.390(0.0)$ & $2.350(0.0)$ \\
\hline$\phi: s \bar{s} 1^{3} S_{1}$ & $1.019(0.0)$ & $1.020(0.0)$ & $1.031(1.9)$ \\
\hline$f_{0}: s \bar{s} 1^{3} P_{0}$ & $1.370(40.0)$ & $1.360(0.0)$ & $1.329(0.0)$ \\
\hline$f_{1}: s \bar{s} 1^{3} P_{1}$ & $1.512(4.0)$ & $1.480(0.7)$ & $1.536(0.4)$ \\
\hline$f_{2}: s \bar{s} 1^{3} P_{2}$ & $1.525(5.0)$ & $1.530(0.0)$ & $1.493(0.4)$ \\
\hline$\phi: s \bar{s} 2^{3} S_{1}$ & $1.680(20.0)$ & $1.690(0.0)$ & $1.852(0.9)$ \\
\hline$\phi: s \bar{s} 1^{3} D_{3}$ & $1.854(7.0)$ & $1.900(0.4)$ & $1.849(0.0)$ \\
\hline$f_{2}: s \bar{s} 2^{3} P_{2}$ & $2.011(69.0)$ & $2.040(0.0)$ & $2.162(0.1)$ \\
\hline
\end{tabular}




\begin{tabular}{|c|c|c|c|}
\hline$\pi: u \bar{d} 1^{1} S_{0}$ & $0.140(0.0)$ & $0.150(1.6)$ & $0.143(0.1)$ \\
\hline$\rho: u \bar{d} 1^{3} S_{1}$ & $0.767(1.2)$ & $0.770(0.0)$ & $0.788(0.1)$ \\
\hline$b_{1}: u \bar{d} 1^{1} P_{1}$ & $1.231(10.0)$ & $1.220(0.0)$ & $1.397(2.6)$ \\
\hline$a_{0}: u \bar{d} 1^{3} P_{0}$ & $1.450(40.0)$ & $1.090(1.0)$ & $1.507(0.0)$ \\
\hline$a_{1}: u \bar{d} 1^{3} P_{1}$ & $1.230(40.0)$ & $1.240(0.0)$ & $1.573(0.8)$ \\
\hline$a_{2}: u \bar{d} 1^{3} P_{2}$ & $1.318(0.7)$ & $1.310(0.0)$ & $1.309(0.0)$ \\
\hline$\pi: u \bar{d} 2^{1} S_{0}$ & $1.300(100.0)$ & $1.300(0.0)$ & $1.535(0.1)$ \\
\hline$\rho: u \bar{d} 2^{3} S_{1}$ & $1.465(25.0)$ & $1.450(0.0)$ & $1.774(1.6)$ \\
\hline$\pi_{2}: u \bar{d} 1^{1} D_{2}$ & $1.670(20.0)$ & $1.680(0.0)$ & $1.871(1.0)$ \\
\hline$\rho: u \bar{d} 1^{3} D_{1}$ & $1.700(20.0)$ & $1.660(0.0)$ & $1.986(2.2)$ \\
\hline$\rho_{3}: u \bar{d} 1^{3} D_{3}$ & $1.691(5.0)$ & $1.680(0.0)$ & $1.711(0.1)$ \\
\hline$\pi: u \bar{d} 3^{1} S_{0}$ & $1.795(10.0)$ & $1.880(0.5)$ & $2.169(9.4)$ \\
\hline$\rho: u \bar{d} 3^{3} S_{1}$ & $2.149(17.0)$ & $2.000(0.5)$ & $2.335(0.8)$ \\
\hline$\rho_{4}: u \bar{d} 1^{3} F_{4}$ & $2.037(26.0)$ & $2.010(0.0)$ & $2.036(0.0)$ \\
\hline$\pi_{2}: u \bar{d} 2^{1} D_{2}$ & $2.090(29.0)$ & $2.130(0.0)$ & $2.372(0.6)$ \\
\hline$\rho_{3}: u \bar{d} 2^{3} D_{3}$ & $2.250(45.0)$ & $2.130(0.1)$ & $2.307(0.0)$ \\
\hline$\rho_{5}: u \bar{d} 1^{3} G_{5}$ & $2.330(35.0)$ & $2.340(0.0)$ & $2.311(0.0)$ \\
\hline$\chi^{2}$ & 0.0 & 84.5 & 104.7 \\
\hline
\end{tabular}

